\title{
MASTER \\ IS-T-832 \\ A STRUCTURAL STUDY OF HIGH TEMPERATURE METAL-RICH TITANIUM SULFIDE PHASES
}

\author{
James Patrick Owens
}

Based on a Ph.D. thesis submitted to Iowa State University

Ames Laboratory, USDOE Iowa State University

Ames, Iowa 50011
This report was prepared as an account of work sponsored by the United States Government. Neither the United States nor the United States Department of Energy, uUt any of their employees, nor any of their contractors, subcontractors, or their employees, makes any warranty, express or implied, or assumes any legal liability or responsibility for the accuracy, completeness or usefulness of any information, apparatus, product or process disclosed, or represents that its use would not infringe privately owned rights.

Date Transmitted: January 1979

PREPARED FOR THE U.S. DEPARTMENT OF ENERGY UNDER CONTRACT NO. W-7405-eng-82 


\section{DISCLAIMER}

This report was prepared as an account of work sponsored by an agency of the United States Government. Neither the United States Government nor any agency Thereof, nor any of their employees, makes any warranty, express or implied, or assumes any legal liability or responsibility for the accuracy, completeness, or usefulness of any information, apparatus, product, or process disclosed, or represents that its use would not infringe privately owned rights. Reference herein to any specific commercial product, process, or service by trade name, trademark, manufacturer, or otherwise does not necessarily constitute or imply its endorsement, recommendation, or favoring by the United States Government or any agency thereof. The views and opinions of authors expressed herein do not necessarily state or reflect those of the United States Government or any agency thereof. 


\section{DISCLAIMER}

Portions of this document may be illegible in electronic image products. Images are produced from the best available original document. 
This report was prepared as an account of wrok spnnsored by the United States Government. Neither the United States nor the United States Department of Ener- . gy, nor any of their employees, nor any of their contractors, subcontractors, or their employees, makes any warranty, express or implied, or assumes any legal liability or responsibility for the accuracy, completeness, or usefulness of any information, apparatus, product or process disclosed, or represents that its use would not infringe privately owned rights.

Available from: National Technical Information Service U. S. Department of Commerce P.O. Box 1553

Springfield, VA 22161

Price: Microfiche $\$ 3.00$ 
A structural study of high temperature metal-rich titanium sulfide phases

by

\section{James Patrick Owens}

A Dissertation Submitted to the

Graduate Faculty in Partial Fulfillment of

The Requirements for the Degree of

DOCTOR OF FHILOSOPHY

Department: Chemistry

Major: Physical Chemistry

Approved:

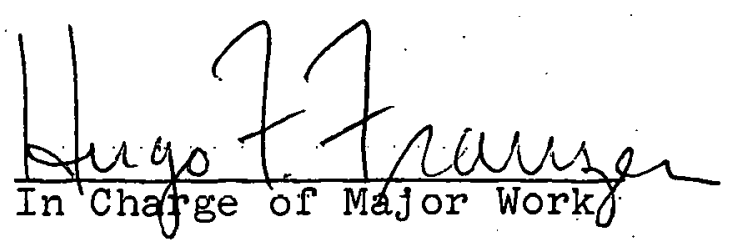

$\frac{\text { Cole th thelve }}{\text { For the Major Department }}$

For the Graduate College.

Iowa State University

Ames, Iowa

1978 
TABLE OF CONTENTS

Abstract

PART I. AN EXPERIMENTAL STUDY OF THE METAL-

Page RICH TITANIUM SULFUR SYSTEM AT HIGH TEMPERATURE

I. INTRODUCTION

A. Sample Preparation ' 2

B. X-Ray Diffraction Techniques 4

C. The Phase Problem 5

II. THE METAL-RICH TITANIUM SULFUR SYSTEM 15

A. Introduction ib

B. Experimental Investigation 17

C. Structure Solution of $\mathrm{TI}_{2} \mathrm{~S}$

D. Structure Solution of $\mathrm{TI}_{8} \mathrm{~S}_{3}$

PART II. STRUCTURAL CORRELATIONS AND CHEMICAL BONDING

I. INTRODUCTION 40

II. THE $\mathrm{Ti}_{2} \mathrm{~S}$ and $\mathrm{Ti}_{8} \mathrm{~S}_{3}$ STRUCTURES 42

A. Introduction 42

B. 3tructure Descriptions 42

III. STRUCTURAL CLASS 51

A. Introduction. 51

B. Structures Forming this Structural Claso

C. Physical Properties $\quad 55$

D. Common Structural Features 56 
1. J. C. Slater, J. Chem. Phys. 4I, 3199 (1964).

2. L. Pauling, The Nature of the Chemical Bond, 3rd edition (Cornell University Press, New York, 1960).

3. R. E. Rundle, Acta Crystallog. I, 180 (1948).

4. L. Brewer, "Thermodynamic Stability and Bond Character in Relation to Electronic Structure and Crystal

Structure", in Electronic Structure and Alloy Chemistry of the Transition Elements, edited by P. A. Beck (Interscience, New York, N.Y., 1963).

5. H. F. Franzen, J. Inorg. Nucl. Chem. 28, 1575 (1966).

6. S. L. Altmann, C. A. Coulson and W. Hume-Rothery, Proc. Roy. Soc., Ser. A 240, 145 (1957). 
IV. STRUCTURAL ASPECTS OF THE METAL P.C.P.

A. A Relationship Between the Me and X P.C.P. 7.0

B. Packing of the Metal P.C.P. 73

C. Me-X Bonds in P.C.P. I through VIII 78

D. The Same P.C.P. for Different Metals 81

E. Different P.C.P. for the Same Metal 82

F. Occurrence of the Me P.C.P. and the Me/X Ratio

V. PARTIAL COORDINATION POLYHEDRA AND INTERATOMIC DISTANCES

A. Introduction 85

B. Variation in Interatomic Distances 86

C. Nonmetal Capping Atoms for P.C.P. II and IV

D. Differences in Me-X Bond Distances for Units VI and VII

VI. STRUCTURAL IMPLICATIONS OF THE PARALLEL TRIGONAL PRISM

A. Structural Limitations 105

B. A Possible Method for Structural Solution 110

C. The $\mathrm{Fe}_{2} \mathrm{P}, \mathrm{CO}_{2} \mathrm{P}, \mathrm{Fe}_{2} \mathrm{As}, \mathrm{V}_{12} \mathrm{P}_{7}$, and $\mathrm{Rh}_{4} \mathrm{P}_{3}$ Structure Types

VII. QUALITATIVE BONDING CONSIDERATIONS 121

A. Review of Qualitative Bonding Considerations 121

B. Altmann, Coulson, and Hume-Rothery 124

C. Application to the Metal P.C.P. .. 133

D. Qualitative Bonding Considerations and
Structural Features

E. Qualitative Model and Polnt Group Symmetry 152 
VIII. STRUCTURAL COMPARISONS

A Introduction 162

B. $\mathrm{TI}_{2} \mathrm{~S}$ and $\mathrm{TI}_{8} \mathrm{~S}_{3}$

C. $\mathrm{Nb}_{21} \mathrm{~S}_{8}$ and $\mathrm{Nb}_{14} \mathrm{~S}_{5}$

D. $\mathrm{Nb}_{21} \mathrm{~S}_{8}$ and $\mathrm{Ti}_{8} \mathrm{~S}_{3}$

IX. FUTURE WORK . . . 188

X. BIBLIOGRAPHY 189 
Table 1. Phases identified in arc-melted titanium sulfide samples

Table 2. Phase identification and transformation of titanium sulfide samples annealed at high temperature

Table 3. Guinier X-ray powder diffraction data for $\mathrm{T} \perp \mathrm{X}$.

Table 4. Final atomic parameters for $\mathrm{T}_{8} \mathrm{~S}_{3}$. All atoms occupy four-fold positions (1) $x$, $0, z$ of space group $\mathrm{C} 2 / \mathrm{m}$

Table 5. The nearest neighbors and their interatomic distances in $\mathrm{Ti}_{8} \mathrm{~S}_{3}$. (Deviations for Ti-S and Ti-Ti distances are $0.050 \AA$ and $0.035 \AA$, respectively)

Table 6. Comparison of phase values calculated by MULTAN techniques with the refined phase values for $\mathrm{Ti}_{8} \mathrm{~S}_{3}$. (Phase in degrees )

Table 7. Interatomic distances for $\mathrm{T}_{2} \mathrm{~S}$. All distances ( $\mathrm{T} 1-\mathrm{T} 1$ ) less than $3.30 \AA$ are listed

Table 8. Structural class

Table 9. Frequency of occurrence of metal Partial Coordination Polyhedra (P.C.P.) in the structures of the layered class

Table 10. Variability of interatomic distances for P.C.P. IV

Table 11. Variability of interatomic distances (A) for $P: C: P$. II

Table 12. Me-X interatomic distances for the trigonal prism 
Table 13. Relat1ve Interatomic distances to polyhedral and capping nonmetal atoms of P.C.P. II and IV

Table 14. Differences in Me-X interatomic distances for P.C.P. VI and VII

Table 15. Symmetry relationship between the $\mathrm{C}_{3 \mathrm{~V}}$ and $D_{3 h}$ point groups

Table 16. Difference in interatomic distances for atoms with a different number of near nelghbors (Reference Flgure 16)

Tabie 17. Numer1cal comparison of the types of

$$
\text { P.C.P. In } \mathrm{TI}_{8} \mathrm{~S}_{3} \text { and } \mathrm{N}_{21} \mathrm{~S}_{8}
$$




\section{LIST OF FIGURES}

Page

Figure 1. Comparison of $\mathrm{TI}_{2} \mathrm{~S}$ atom positions from superposition techniques with the refined atom positions

Figure 2. The $\mathrm{T}_{2} \mathrm{~S}$ structure

Figure 3. The $\mathrm{TI}_{2} \mathrm{~S}$ structure as the packing of titanium cubes and sulfur trigonal prisms

Figure 4. The $\mathrm{TI}_{8} \mathrm{~S}_{3}$ structure

Figure 5. The metal Partial Coordination Polyhedra (P.C.P.)

Figure. 6. An illustration of the relationship between certain metal partial coordination polyhedra (broken lines) and the nonmetal trigonal prisms (solid Iines)

Figure 7. Interrelationship between face, edge, and corner sharing of metal P.C.P. in one layer (solid lines) with the type of metal P.C.P. in the adjacent layer (broken lines)

Figure 8. Number and orientation of Me-X bonds for metal P.C.P. II through VIII. Numbers of $\mathrm{Me}-\mathrm{X}$ bonds in parentheses

Figure 9. Atom numbering for P.C.P. II and IV in Table 10 and Table 11 , respectively

Figure 10. Atom numbering sequence for paraliel and perpendicular prisms in Table 12

Figure 11. A structural unit as a combination of metal and nonmetal P.C.P. 
Figure 12. Examples of some of the different possible combinations of metal P.C.P. for atoms 3 and 4 of the VI-(11)prism-VI structural unit. The orlentation of P.C.P. In part c) and d) of the figure have not been observed for structures in the layered class

Figure 13. The occurrence of the VII-(II)prism-VI structural unit in the $\mathrm{T}_{2} \mathrm{~S}$ structure

Figure 14. Hexagonal rings formed by the projections of nonmetal atom positions in the two layers of each structure

Figure 15. The stacking of the hexagonal rings in the $\mathrm{Fe}_{2} \mathrm{P}(\mathrm{a})$ and $\mathrm{Co}_{2} \mathrm{P}(\mathrm{b})$ structure types. Orientations for the approximate unit cells are given by the dark lines

Figure 16. An illustration of the differences in the types of P.C.P. (broken lines) for those atoms (solid circles) which are the polyhedral atoms for P.C.P. I (solid lines) in the $\mathrm{TI}_{2} \mathrm{~S}$ and $\mathrm{TI}_{8} \mathrm{~S}_{3}$ structure types

Flgure 17. The $\mathrm{Cr}_{4} \mathrm{As}_{3}$ structure type. Solid Iines indicate the unit cell

Figure 18. The solid lines indicate the $\mathrm{Nb}_{4} \mathrm{As}_{3}$ unit

cell. Broken lines 1llustrate the packing of the $\mathrm{Cr}_{4} \mathrm{As}_{3}$ unit cell to form the $\mathrm{Nb}_{4} \mathrm{As}_{3}$ structure type

Figure 19. Differences in the nature of P.C.P. for $\mathrm{Nb}_{4} \mathrm{As}_{3}$ (part a) and $\mathrm{Cr}_{4} \mathrm{As}_{3}$ (part b) structure types along the horlzontal Iines of connection of the structural unit. Part c) illustrates the near neighbors for As (4) in $\mathrm{Nb}_{4} \mathrm{As}_{3}$

Figure 20. The relationship between P.C.P. I and nonmetal trigonal prisms in $\mathrm{Ti}_{2} \mathrm{~S}$ and $\mathrm{TI}_{8} \mathrm{~S}_{3}$. The types of P.C.P. for the polyhedral metal atoms are indicated 
Figure 21. The $\mathrm{TI}_{2} \mathrm{~S}$ structure as the packing of the structural unit in part a) of Figure
20 . Solid lines 1liustrate P.C.P.
centered about atoms in one layer of
atom positions while broken lines
correspond to P.C.P. for atom
positions in the second layer

Figure 22. The $\mathrm{TI}_{8} \mathrm{~S}_{3}$ structure as the stacking of the structural units in part b), c), and d) of Figure 20

Figure 23. The structural units common to the $\mathrm{Nb}_{21} \mathrm{~S}_{8}$ and $\mathrm{Nb}_{14} \mathrm{~S}_{5}$ structure types. Atoms in the two structural units which have different P.C.P. are identified by Roman numerals corresponding to the type of P.C.P. for the appropriate atoms

Figure 24. $\quad \mathrm{Nb}_{21} \mathrm{~S}_{8}$ structure type 1llustrating the structure unit of part a) in Figure 23. The unit cell is indicated

Figure 25. $\mathrm{Nb}_{21} \mathrm{~S}_{8}$ structure type

Figure 26. $\mathrm{Nb}_{14} \mathrm{~S}_{5}$ structure type. The structural unit in part a) of Figure 23 is indicated. The unit cell is outlined by solid lines

Figure 27. $\mathrm{Nb}_{14} \mathrm{~S}_{5}$

F1gure 28. The different relationship between the metal P.C.P. of type I and the nonmetal trigonal prisms in $\mathrm{Nb}_{21} \mathrm{~S}_{8}$ and $\mathrm{TI}_{8} \mathrm{~S}_{3}$.

The types of metal P.C.P. are indicated 183 
A structural study of high temperature metal-rich titanium sulfide phases ${ }^{1}$

\section{James Patrick Owens}

\section{Under the supervision of H. F. Franzen From the Department of Chemistry Iowa State University}

$\mathrm{Ti}_{2} \mathrm{~S}$ and $\mathrm{Ti}_{8} \mathrm{~S}_{3}$ have been prepared by high temperature annealing tcchriques. The crystal structures of these two phases have been determined from single crystal x-ray diffraction data. Both structures were refined using a fullmatrix least-squares treatment of positional parameters and isotropic temperature factor coefficients.

$\mathrm{Ti}_{2} \mathrm{~S}$ crystallizes with orthorhombic symmetry, space group Pnnm, having unit cell dimensions $\underline{a}=11.367 \AA$, $\underline{b}=14.060 \AA$, and $\underline{c}=3.326 \AA . \mathrm{T}_{2} \mathrm{~S}$ is isostructural with $\mathrm{Ta}_{2} \mathrm{P}$.

$\mathrm{Ti}_{8} \mathrm{~S}_{3}$ crystallizes with monoclinic symmetry, space group $\mathrm{c} 2 / \mathrm{m}, \underline{a}=32.69 \AA, \underline{b}=3.327 \AA, \underline{c}=19.35 \AA, \quad \beta=139.9^{\circ}$ (b- - unique).

${ }^{1} U . S$, DOE Report IS $-T-832$. This work was performed under Contract W-7405-eng-82 with the U.S. Department of Energy. 
$\mathrm{TI}_{2} \mathrm{~S}$ and $\mathrm{TI}_{8} \mathrm{~S}_{3}$ have structural features similar to the features of a large number of metal-rich transition-metal chacogenides and pnictides. These various structure types have been characterized in terms of nonmetal trigonal prismatic coordination polyhedra, elght different metal partial coordination polyhedra, a short $(\approx 3.4 \AA)$ crystallographic axis, two unique layers of atoms containing both metal and nonmetal atom positions, and mirror planes colncident with the two layers of atom positions. The existence of a variety of structures with these structural features has led to their consideration as a unique structural class.

The structural similarities and differences between the structure types of this class have been discussed in detall. Comparison of different structure types emphasized the importance of the metal bonding contribution in understanding the structural features and suggested limitations on qualitative bonding models used to understand the structuralchemical principles underlying structure stability.

The yualitative bonding models of Slater (1), Pauling (2), Rundle (3), Brewer-Engel (4), Franzen (5), and Altmann, Coulson and Hume-Rothery (6) have been used to better understand the structural features of the complex structure types of this structural class. 
PART I. AN EXPER IMENTAL STUDY OF THE METAL-RICH TITANIUM SULFUR SYSTEM AT HIGH TEMPERATURE 


\section{INTRODUCTION}

\section{A. Sample Preparation}

The general preparation techniques used in the study of the metal-rich titanium sulfur system were those commonly utilized by Franzen and co-workers. Details and the advantages of these techniques have been outlined by Conard (I) and Smeggil (2). The various samples were prepared using as starting materials $99.95 \%$ pure titanium metal obtained from the Chicago Development Corporation and $99.999 \%$ purity sulfur from the Gallard-Schleisenger Chemical Manufacturing Corporation. Two slightly different techniques were used for the Initial preparation of the titanium metal. Metal for those samples with $\mathrm{T} 1 / \mathrm{S} \geq 2.0$ was filed from a titanium sheet In a glove bag under an inert argon atmosphere. The initial preparation of the titanium metal used for the samples with $1.25 \leq \mathrm{T} 1 / \mathrm{S} \leq 1.75$ was the technique suggested by Dr. A. Khan of the Ames Laboratory. Titanium metal was reacted with hydrogen gas at $300-400^{\circ} \mathrm{C}$ to form $\mathrm{T} \mathrm{H}_{\mathrm{x}}, 0.5<\mathrm{x}<0.6$. The brittle metal hydride was crushed into a fine powder and the hydrogen removed at $800^{\circ} \mathrm{C}$ to yield powdered titanium metal. Dr. Khan has shown that initial preparation of transition metals in this way provides closer control over the stolchiometry of the final product and more complete reaction of the titanium metal with sulfur during the initial heating of the sample. 
For all of the titanium sulfide samples, the metal and nonmetal were placed in a Vycor reaction tube, the reaction tube evacuated to approximately $10^{-3}$ torr, and the tube sealed under vacuum. All samples were heated in a resistance furnace at 600 to $800^{\circ} \mathrm{C}$ for time periods from one to three weeks. The reaction tubes were opened, glass fragments removed, and the samples pressed into pellets at approximately $30,000 \mathrm{Ib} / \mathrm{In}^{2}$ using a hydraulic press. During the high temperature annealing process, a pellet of the sample was placed in a tungsten crucible that had previously been outgassed at $2000^{\circ} \mathrm{C}$ for one hour. The crucible and samples were heated under a vacuum of $1 \times 10^{-6}$ torr using a Lepel Radio Frequency generator. Samples were normally annealed between 950 and $1750^{\circ} \mathrm{C}$, the particular temperature and annealing time depending on the nature of the sample. Temperature measurements were made using a Leeds and Northrup disappearing filament optical pyrometer. During annealing, the residual pressure was measured with a hot cathode ionization gauge.

An attempt was made to anneal each sample at a temperature slightly below. 1ts melting point, and each sample was usually heated for several four to eight hour perlods. The annealing process for a sample was terminated whon the $X$-ray diffraction patterns taken prior and subsequent to a heating showed no not1ceable change. X-ray powder 
diffraction and single crystal techniques were used to Identify the phases present in each sample.

\section{B. X-Ray Diffraction Techniques}

The X-ray diffraction techniques used in this study involved both single crystal and powder diffraction methods. The theory of $\mathrm{X}$-ray diffraction and the practical application of 1ts use are well-described in the books by Buerger (3), Crystal Structure Analysis, and Stout and Jensen (4), X-Ray Structure Determination. Both techniques were used to help characterize the phases present in the various samples that were prepared. Single crystal techniques were used to provide Intensity data for the structural solution of $\mathrm{TI}_{2} \mathrm{~S}$ and $\mathrm{T}_{8} \mathrm{~S}_{3}$

\section{i. Gulnier diffraction techniques}

A Guinier $\mathrm{X}$-ray camera with an approximate radius of $80 \mathrm{~mm}$. was used to obtain powder diffraction films for most samples. The Guinier camera provides some advantages over the more common Debye-Scherrer camera. For example, Guinier films provide better resolution of low angle IInes characteristic of $K \alpha_{1}$ radiation and the refiections are unshifted by absorption and eccentricity, phenomena which Increase the errors in the Debye-Scherrer technique. Guinier fllms for each sample were obtained using copper $K \alpha_{1}$ radiation and silicon $(a=5.4301 \AA)$ as an internal standard. 
The various films were read and $\sin ^{2} \theta$ values were calculated for comparison with $\sin ^{2} \theta$ values for known phases within a particular system. As samples were annealed at different temperatures, the Guinier diffraction films were compared to those for previous samples to determine if a sample was undergoing phase change. When avallable, diffraction films were compared directly to films of known phases.

\section{Single crystal techniques}

Utilization of single crystal techniques to determine the reciprocal lattice and to provide intensity data for structural solution of a previously unknown phase have been described by Conard (1) and Smeggil (2). The techniques they describe were used in the intensity data collection for the structural solution of $\mathrm{T}_{2} \mathrm{~S}$ and $\mathrm{T}_{8} \mathrm{~S}_{3}$. Single crystal techniques were also used to identify phases which were present in various samples in a concentration too small to be observed in Guinler diffraction rllms. Orten single crystal techniques were used to identify a second phase present in samples for which powder diffraction films Indicated the presence of only a single phase.

\section{The Phase Problem}

The orlgin of the phase problem that must be solved during the structure determination of any crystaline solid 
with a previously unknown structure type is easy to understand, while the means of solving the phase problem are varled and complex. During collection of crystallographic data by single crystal techniques, the experimentally measured quantity is the intensity, $I_{h}$, for a particular reflection, $\underset{\sim}{h}$. The structure factor, $\mathrm{F}_{\mathfrak{n}}$, corresponding to the same reflection is given by

$$
\begin{aligned}
F_{\underset{\sim}{h}}=\sum_{j} f_{j} & \exp \left(2 \pi 1\left(\underset{\sim}{h} \cdot{\underset{\sim}{j}}_{j}\right)\right] \\
= & \sum_{j} f_{j}\left[2 \pi 1\left(h x_{j}+k y_{j}+l z_{j}\right)\right]
\end{aligned}
$$

where the summation is over all of the $f$ scattering centers. The intensity can be related to the structure factor by the expression

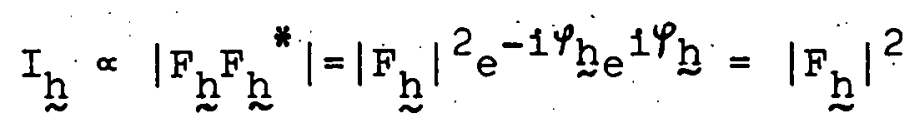

which indicates that the phase of the structure factor, $\Psi_{\underline{h}}$, for a reflection is not directly obtained in the experimental measurement of $I_{h}$.

\section{Patterson techniques}

Patterson techniques have traditionally provided one of the most successful methods for the solution of the crystallographlc phase problem, 1 .e.., for the determination of the phase angle assoclated with a given reflection. The theory and utility of the method have been thoroughly discussed by Buerger (5), while the book by Stout and Jensen 
(4) provides an excellent introduction to this method for chemists starting work on a crystallographic problem. Woolfson (6) expressed the Patterson function as

$$
P(\mathfrak{r})=\frac{I}{V} \underset{\sim}{\underset{\sim}{h}}\left|F_{\underline{\alpha}}\right|^{2} \exp [-2 \pi 1(h \cdot r)]
$$

where

$$
\underline{r}=\mathrm{xa}+\mathrm{yb}+\mathrm{zc}
$$

and

$$
\underline{\sim}=\mathrm{ha}^{*}+\mathrm{kb}^{*}+1 \mathrm{~g}^{*}
$$

and shows that the Patterson function is the self-convolution of the electron density function. The relationship between the Patterson function and the electron density allows a physical interpretation of the Patterson function as the representation of the vector density between two infinftesimal regions of electron density separated by the given vector and summed over a unit cell. A Patterson map Is thus a representation of all vectors between the infinitesimal regions of electron density associated with the same or different atoms of the structure.

The integrated magnitude associated with a single interatomic vector is proportional to the sum of the products of the numbers of electrons in the atoms separated by the vector considered. If a structure contains $n$ atoms, or regions of electron density, there are $n^{2}-n$ Patterson vectors (excluding vectors from one part of an atom to nelghboring regions of the same atom (origin peak)). A Patterson map can be considered as $n$ images of the structure all 
superimposed on one unit cell. The complexity of a Patterson map is due to three contributing factors:

1) The high density of $n^{2}-n$ Patterson peaks within one unit cell (1260 for $\mathrm{TI}_{2} \mathrm{~S}$ ) causes considerable overlap of individual peaks.

ii) Since atoms are not point sources of electron density, Patterson peaks have a finfte size which adds considerably to the overlap of such peaks. In fact, the size of a Patterson peak associated with each atom is twice the size of 1ts corresponding electron density.

1i1) Because the Fourier expansion of the Patterson function includes only a finite number of terms, there can be a rippling effect for each Patterson peak. Overlap of such ripples for two atoms can further complicate a Patterson map by giving additional (false) peaks.

One technique often used to resolve the Images of the structure in the Patterson map is the superposition or vector-shift method (5). This method has its greatest utility when applied to layered structures. In this case all of the Patterson vectors lie in parallel planes, thus reducing the problem to one of two dimensions. This procedure uses two Identical copies of the Patterson map, map $A$ and map $B$. In principle, if a Patterson peak of map $B$ corresponding to an atom position (1..e., from an atom at the origin to the atom position in question) is placed over the 
origin of map A, those peaks that overlap in the two maps provide an image of the structure and 1ts inverse. If this procedure is repeated for each peak in map B that corresponds to an atom position, repetition of the same structural image occurs. Ideally, comparison of several such superposition maps allows recognition of the structure.

For the practical application of this method there are two basic problems to be overcome. First, the discussion above assumes that those Patterson peaks which actually correspond to atom positions are known. Although this information is not known, there are techniques that can be used to increase the probability that a chosen peak actually corresponds to an atom position. Even a complex map will often contaln a few well resolved peaks with magnitude roughly equal to an expected vector between two heavy atoms. The probability of such a peak corresponding to an atom position is much larger than for a general peak. The space group symmetry and expected structural chemistry can also be used to help choose such peaks. The second problem is concerned with the question of actual peak location in the large positive areas of the Patterson map. Seldom can each of the component peaks of such an area be resolved, but a general area of the Patterson map, rather than a specific point, can often be assoclated with possible atom positions. There must be some flexibility in deciding whether two peaks 
overlap in superposition maps. Comparison of several superposition maps provides some possible atom positions that are well pinpointed, while others occur in larger, less welldefined regions of the unit cell, as Indicated in Figure I in section II below.

The following outline summarizes the general procedures which. were used to solve the $\mathrm{TI}_{2} \mathrm{~S}$ structure and which were used In the attempted solution of the $\mathrm{TI}_{8} \mathrm{~S}_{3}$ structure:

1) A starting set of peaks in the Patterson map that had a high probability of corresponding to atom positions was chosen.

11) Superposition maps were prepared for each of the peaks of the starting set. The superposition maps were then compared to find peaks and groups of peaks that recurred in the various maps.

111). If a particular superposition map did not contain the recurring features common to the majority of the maps, that superposition map was discarded and the corresponding origin peak removed t'rom the starting set.

1v) Additional superposition maps were made for those peaks that were absent in the starting set but which kept recurring in the various maps.

v) The resulting group of peaks formed a trial structure which was continuously analyzed in terms of space group symmetry, packing of atoms, and the expected structural chemistry for the titanium sulfur system. Ideally, as the 
various peaks are tested, more atoms are located, and the trial structure becomes the true structure. In the actual application of this procedure, a point was reached where a large part of the trial structure corresponded to the true structure while additional atom positions required testing by Fourier synthesis.

\section{MULTAN techniques}

Direct methods use definite mathematical relationships which are usually based on inequality and probability considerations to provide a phasing model. There are a large number of direct methods that can be used for phase determination. The MULTAN techniques will be considered in some detail since their application was successful in solving the structure: of $\mathrm{Ti}_{8} \mathrm{~S}_{3}$.

Between 1968 and 1971, a series of papers by Germain, Main, and Woolfson $(7,8,9)$ appeared. In this series they described a method and designed a computer program for the solution of the phase problem for both centrosymmetric and noncentrosymmetric structures. Their methods were an extension of the phase determining formulas of Karle and Karle (10) and provide solutions for the common problems associated with symbolic addition procedures. These techniques, called MULTAN, have been used widely in the past few years with notable success. The highly automated 
MULTAN computer program has often provided rapid solution of complex crystallograph1c problems.

The MULTAN technique is based upon two basic results derlved by Karle and Karle (10). The slgma 2 relat1onsh1ps

$$
\varphi_{\underline{\underline{h}}} \approx \varphi_{\mathfrak{h}^{\prime}}+\varphi_{\underline{\underline{h}}-\underline{h}^{\prime}}
$$

indicate that the phase of a reflection, $\underset{\sim}{ }$, can be determined if the phases for the reflections $h^{\prime}$ and $h-h^{\prime}$ are already known. The accuracy of this relationship increases for larger values of the normalized structure factors, $\mathbb{E}_{\underset{\sim}{ }}$, assoclated with the various reflections. The second formula, called the tangent formula, is represented by

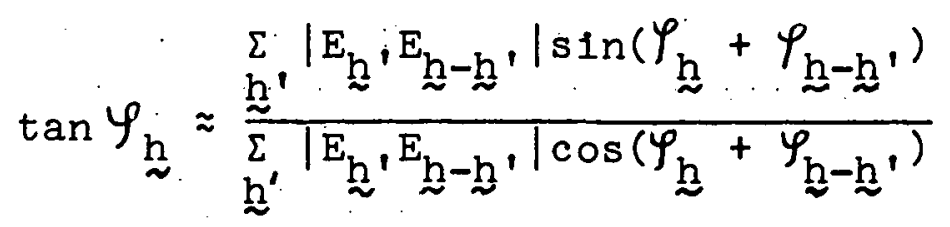

where the summation is over all reflections of known phase which have relatively high values of $E_{\underline{h}}$, and $E_{\underline{h}-\underline{h}}$, (normally only reflections with $\left|E_{h}\right| \geq 1.50$ are included). Both of the formulas in equations (6) and (7) are closely related and can be derlved from both algebraic and probabllity considerations (10).

The use of these expressions in determining the phase of a reflection requires a starting set of reflections of known phase. Ideally, the starting set can be used to continue the phase determining process until the phases are known for all of the strong reflections. As in the use of 
Sayre's triple product relations for centrosymmetric structures, however, an early mistake in phase determination causes the phases of a large number of reflections to be wrong, ylelding an incorrect trial structure. Since initially there are seldom enough phases known to allow complete phase assignment, certain reflections are assigned symbols to represent their phases, and unknown phases are determined in terms of the symbols.

One of the most important aspects of the MULTAN technique was the inclusion of a method for finding the best possible starting set of phases. The method depends upon the reliability of a particular phase determination, $\alpha_{h}$, which is given by the expression

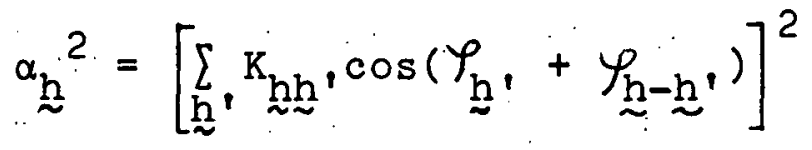

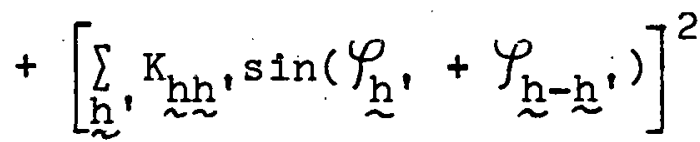

In this expression $\alpha_{\mathfrak{h}}$ depends directly on the phase values whlch are not known when the starting set of reflections is chosen. It is possible, however, to estimate $\alpha_{h}$ by the expression

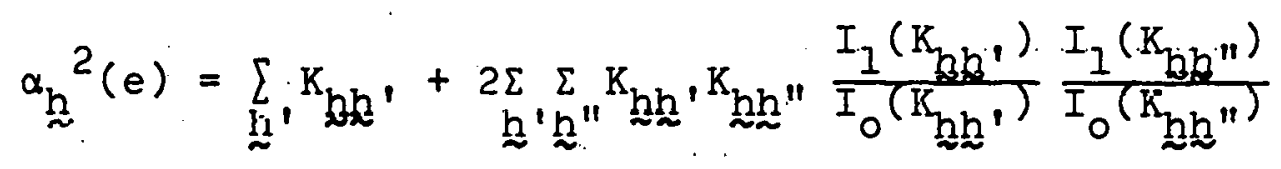

( $I_{1}$ and $I_{0}$ are Bessel functions) 


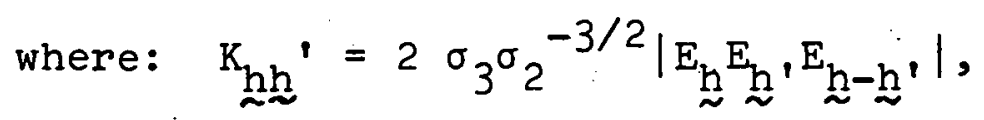

$\sigma_{n}=\sum_{j=1}^{N} z_{j}^{n}\left(z_{j}\right.$ is the atomic number of the $j$-th atom)

$$
\frac{I_{1}(K)}{I_{0}(K)} \approx 0.5658 \mathrm{~K}+0.1304 \mathrm{~K}^{2}+0.0106 \mathrm{~K}^{3}+\cdots
$$

The estimated reliability, $\alpha_{\underset{\sim}{h}}(e)$, for the phase determination of a reflection does not depend upon knowing the phases, but is proportional to the magnitude of the $E_{\underline{h}}$ 's involved in any triple product as well as proportional to the number of triple product relationships for a particular reflection. The best starting set is determined by the step by step elimination of those reflections having low values for $\alpha_{\mathfrak{h}}(e)$. The remalning reflections, those necessary to define the origin, to define the enantiomorph, and to specify a number of reflections requiring phase symbol assignment, are those which phases are known with high reliability and which are related by the sigma 2 relationships to a large number of other reflections.

The specific application of these techniques is considered in the discussion of the $\mathrm{TI}_{8} \mathrm{~S}_{3}$ structure solution in section. II below. 
II. THE METAL-RICH TITANIUM SULFUR SYSTEM

\section{A. Introduction}

\section{Survey of metal-rich titanium sulfides}

The investigation of the titanium sulfur system began with the 1937. work of Biltz, Ehrlich, and Meisel (11). Since that time there have been several investigators who have studied this system with varying degrees of thoroughness. With the advent of the techniques of high temperature chemistry, sample-container interactions, which had caused contradictory phase characterization among early investigators, were minimized. Conard (1) summarized the work of the early investigators and discussed those cases where sample-container. Interactions had caused problems in phase characterization.

During his investigation of the vaporization of TiS, Franzen (12) reported the presence of a new metal-rich titanium sulfide with nominal composition, $\mathrm{TI}_{2} \mathrm{~S}$. Using Franzen's sample, Stone (13) attempted the crystallographic characterization of this new solid phase. A least-squares fit of Guinier powder diffraction lines for this sample,gave approximate lattice parameters of $\underline{a}=11.35, \underline{b}=14.06$, and $\underline{c}=3.32 \AA$. Stone also determined the space group symmetry to be either Pnn2 (noncentrosymmetric) or Pnnm (centrosymmetric). Combustion analysis ylelded $\mathrm{S} / \mathrm{T} 1=0.498$, and 
density measurements provided an average density of $4.80 \mathrm{~g} \mathrm{~cm}^{-3}$. This density corresponded to $11.97 \mathrm{TI}_{2} \mathrm{~S}$ units per unit cell.

In 1958, Bartram (14) 1dentifled a new titanium sulfide phase of nominal composition, $\mathrm{TI}_{6} \mathrm{~S}$, which he described as having a unit cell closely related to that of titanium metal. The $\mathrm{Ti}_{6} \mathrm{~S}$ unit cell was hexagonal with lattice parameters $\underline{a}=2.9669 \pm 0.0004$ and $\underline{c}=14.495 \pm 0.005 \AA$ where the $\underline{a}$-axis is slightly larger than the corresponding a-axis of the metal and the c-axis is approximately three times the c-axis of the metal. Bartram confirmed the presence of sulfur in the sample and proposed at least partial ordering of sulfur in the titanium lattice to account for the approximate tripling of the $\underline{c}-a x i s$.

In 1969, Eremenko and Listovnich11 (15) reported the preparation of $\mathrm{TI}_{3} \mathrm{~S}$ and determined that the space group was tetragonal with lattice parameters of $\underline{a}=9.952$ and $\underline{c}=4.89 \AA$. Based on powder diffraction data, they suggested that $\mathrm{TI}_{3} \mathrm{~S}$ was isostructural with $\mathrm{TI}_{3} \mathrm{P}$.

\section{Purpose of this investigation}

The purpose of the research described in this chapter was to determine the structure of $\mathrm{TI}_{2} \mathrm{~S}$, to duplicate the reported work on $\mathrm{TI}_{3} \mathrm{~S}$ and $\mathrm{TI}_{6} \mathrm{~S}$, and to systematically Investigate the T1-S system for the presence of other poss1ble metal-rich phases which are stable at h1gh temperature. 
B. Experimental Investigation

Three different sets of titanium sulfide samples were prepared using different experimental conditions. Table 1 Iists the set of samples which were prepared by arc-melting pellets of $T 1 S$ and titanium metal combined in the mole ratio corresponding to the approximate stoichiometry indicated in the table. The arc-melted pellets were annealed for elght

Table 1. Phases identified in arc-melted titanium sulfide samples

Nominal Sample

Composition
Phases

Present
Identification Method

$\begin{array}{lc}\mathrm{T}_{3} \mathrm{~S} & \mathrm{~T}_{8} \mathrm{~S}_{3}, \mathrm{TI}_{2} \mathrm{~S} \\ \mathrm{~T}_{4} \mathrm{~S} & \mathrm{~T} 1 \\ \mathrm{~T}_{5} \mathrm{~S} & \mathrm{~T} 1 \\ \mathrm{~T}_{6} \mathrm{~S} & \mathrm{~T} 1 \\ \mathrm{~T} 1_{7} \mathrm{~S} & \mathrm{~T} 1 \\ \text { a oorrcsponds to single crystal techniques. } \\ \text { b corresponds to powder diffraction techniques. }\end{array}$

to ten hours at the relatively low temperature of $1125^{\circ} \mathrm{C}$, since attempted annealing at higher temperatures resulted in melting of samples. Only the $\mathrm{TI}_{3} \mathrm{~S}$ sample yielded diffraction 
patterns indicating the presence of a phase other than titanium metal. Single crystal work on this particular sample confirmed the presence of $\mathrm{TI}_{2} \mathrm{~S}$ and Indicated the presence of a new titanium sulfide phase which was subsequently identifled as $\mathrm{Ti}_{8} \mathrm{~S}_{3}$.

A second set of samples, Ilsted in Table 2, was prepared from the elements in the manner discussed in the Introduction.

Table 2. Phase identification and transformation of titanium sulfide samples annealed at high temperature.

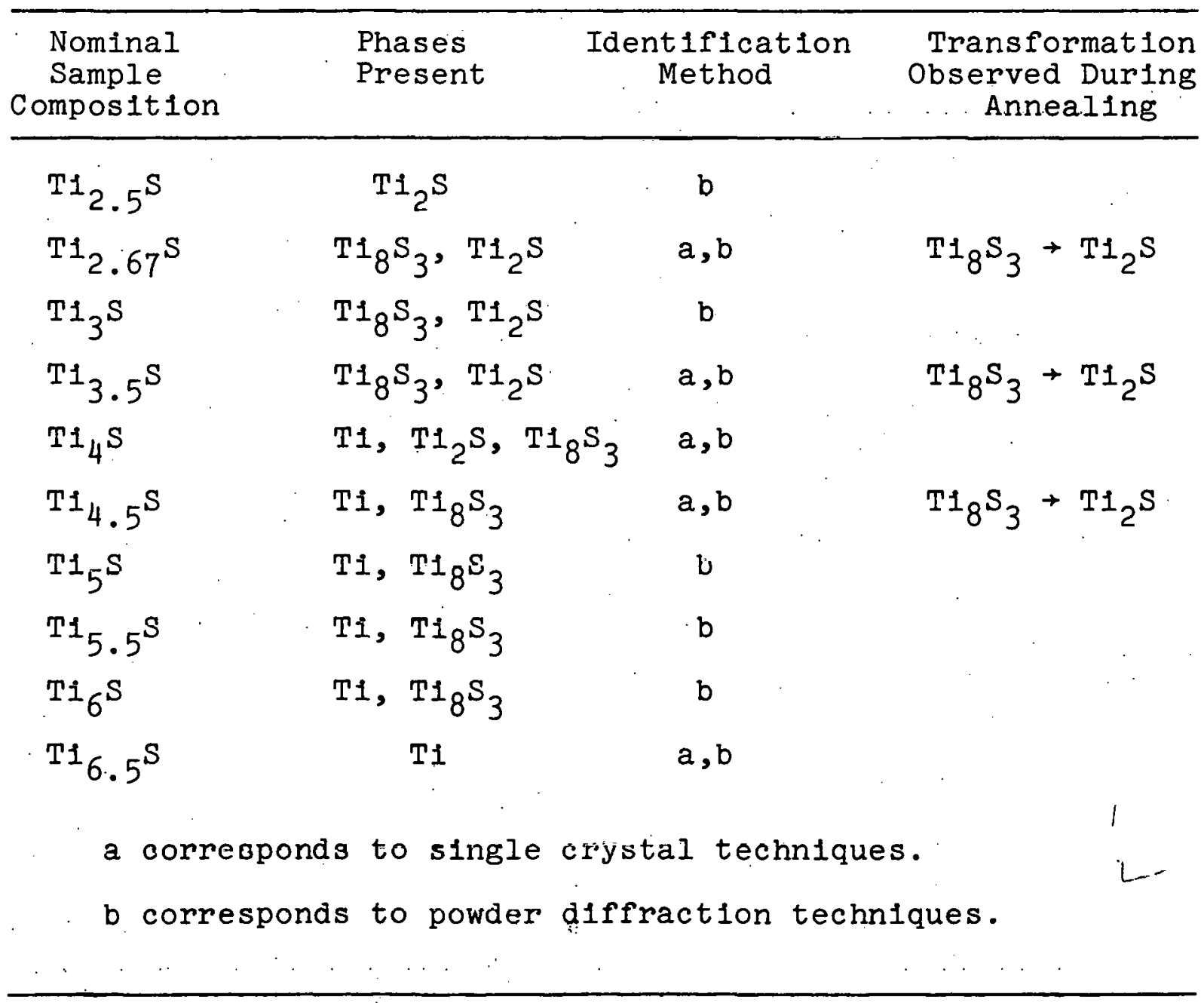


The phases present in each of these samples were identified by powder diffraction and single crystal techniques. For each of the different samples the total heating time at $1150^{\circ} \mathrm{C}$ varled depending on the behavior of the individual samples. For example, after eight hours of annealing, Guinier diffraction films for the $\mathrm{TI}_{3.5} \mathrm{~S}$ sample indicated the presence of $\mathrm{TI}_{2} \mathrm{~S}$ and $\mathrm{TI}_{8} \mathrm{~S}_{3}$. After approximately 200 hours of heating, the presence of only $\mathrm{TI}_{2} \mathrm{~S}$ was indicated by X-ray diffraction techniques. The decomposition of $\mathrm{Ti}_{8} \mathrm{~S}_{3}$ at $1150^{\circ} \mathrm{C}$ was also observed for the $\mathrm{T}_{2} .67^{\mathrm{S}}$ and $\mathrm{T} 1_{4.5} \mathrm{~S}$ samples. Even after most of the $\mathrm{TI}_{8} \mathrm{~S}_{3}$ had decomposed, Guinier diffraction films for the samples $\mathrm{TI}_{2} .67^{\mathrm{S}}$ and $\mathrm{T}_{3} .5^{\mathrm{S}}$ gave no evidence for the presence of titanium metal. It is possible that titanium dissolved into the tungsten container.

A thorough single-crystal examination was conducted on. the $\mathrm{TI}_{3} \mathrm{~S}, \mathrm{TI}_{4} .5 \mathrm{~S}$, and $\mathrm{Ti}_{6} \mathrm{~S}$ samples. No evidence was found to confirm the existence of the $\mathrm{TI}_{3} \mathrm{~S}$ phase reported by Eremenko and Listovnichi1 (15) or the $\mathrm{TI}_{6} \mathrm{~S}$ phase reported "by Bartram (14). The examination of the $\mathrm{TI}_{4} .5^{\mathrm{S}}$ sample gave no indication for the presence of a titanium sulfide phase corresponding to the $\mathrm{Zr}_{9} \mathrm{~S}_{4}$ phase recently characterized by Chen and Franzen (1.6). The possible exigtence of these phases certainly cannot. be ruled out, but under the conditions of attempted preparation these particular phases do not appear to be stable. 
A preliminary investigation was conducted on samples of

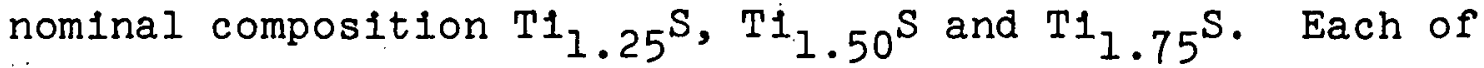
these samples exhibited similar behavior at high temperature. Guinier diffraction films for the $\mathrm{TI}_{1.25} \mathrm{~S}$ sample heated at $1500-1600^{\circ} \mathrm{C}$ indicated the presence of an unidentified phase, $\mathrm{T} 1_{\mathrm{x}} \mathrm{S}$. The observed $\sin ^{2} \theta$ values for this phase are Iisted In Table 3. Although the diffraction films gave no evidence for the presence of TIS, only single crystals of TIS were obtained from this sample. After annealing at $1750^{\circ} \mathrm{C}$, diffraction films of the $\mathrm{T} i_{1.25}$ sample confirmed the presence of both $\mathrm{TI}_{\mathrm{X}} \mathrm{S}$ and $\mathrm{TIS}$. Characterization of the $\mathrm{T}_{\mathrm{X}} \mathrm{S}$ phase will require further investigation.

Table 3. Gulnier X-ray powder diffraction data for TI $\mathrm{S}$

\begin{tabular}{lrl}
\hline $\begin{array}{l}\text { Reiative } \\
\text { Intensity }\end{array}$ & $\begin{array}{c}\sin ^{2}(\theta) \text { observed } \\
x .104\end{array}$ & $\theta$ (observed) \\
\hline M & 726.1 & 15.63 \\
W & 877.2 & 17.23 \\
VW & 1169.0 & 20.00 \\
VW & 1202.0 & 20.29 \\
S & 1309.0 & 21.21 \\
VW & 2040.0 & 26.85 \\
VW & 2192.0 & 27.92 \\
VW & 2308.0 & 28.72 \\
VW & 3044.0 & 33.49 \\
W & 3514.0 & 36.36 \\
W & 4505.0 & 42.16 \\
\hline
\end{tabular}




\section{Structure Solution of $\mathrm{TI}_{2} \mathrm{~S}$}

1. Acknowledgement

The structural study of the $\mathrm{TI}_{2} \mathrm{~S}$ phase was the combined effort of the author and Dr. B. R. Conard, whose Ph.D. thesis contains a detailed discussion of the $\mathrm{TI}_{2} \mathrm{~S}$ structure determination. This section is a description of the author's contribution to the structural solution for $\mathrm{TI}_{2} \mathrm{~S}$.

2. Preparation and data collection

A sample of intial composition $\mathrm{Ti}_{2} \mathrm{~S}$ was prepared by annealing at $1590^{\circ} \mathrm{K}$. The annealing was repeated until powder diffraction films indicated no further changes in the sample. The resulting product was extremely hard, brittle, and characterlied by high metallic luster. Many of the small pleces of the sample chlpped from the tungsten crucible were characterized by well-defined faces and sharp edges indicative of well formed single crystals.

A single crystal was chosen from this sample and aligned with the crystallographic $\underline{c}$-axis coincident with the rotation axis of a Weissenberg camera. Rotation and Weissenberg films were taken for the first four layers using molybdenum $K_{\alpha}$ radiation. Reciprocal lattice plots indicated that the lattice parameters were the same as those reported by Stone (13) and confirmed that the point group symmetry for the unit cell was orthorhombic. The conditions for reflection 
were determined to be:

$$
\begin{array}{ll}
\text { hkl: } & \text { no conditions } \\
\text { Okl: } & k+l=2 n \\
\text { hol: } & h+l=2 n \\
\text { hko: } & \text { no conditions } \\
\text { hoo: } & h=2 n \\
\text { OkO: } & k=2 n \\
\text { Ool: } & l=2 n
\end{array}
$$

These reflection conditions were consistent with the two orthorhombic space groups Pnn2 and Pnnm.

The assumption was made that the $\mathrm{TI}_{2} \mathrm{~S}$ structure corresponded to the centrosymmetric space group Pnnm. The short c-axis of $3.32 \AA$ and packing considerations for the atoms combined to limit the atom positions of the assumed Pnnm space group whlch could be occupled by titanium or sulfur. If the general atom positions, $8(\mathrm{~h})$, were occupled, an atom in the $(x, y, z)$ position would imply a second atom at $(x, y, \bar{z})$. The short $\underline{c}$-axis implied that atoms occupying these two positions would be too close together for any value of $z$ different from $z=0$ or $z=1 / 2$. These considerations indicated that the $4(\mathrm{~g})$ atom positions $(\mathrm{x}, \mathrm{y}, 0 ; \overline{\mathrm{x}}, \overline{\mathrm{y}}, 0 ; 1 / 2+\mathrm{x}, 1 / 2-\mathrm{y} ; 1 / 2$; $1 / 2-x, 1 / 2+y, 1 / 2)$ of the Pnnm space group were the most general positions that could be occupled by titanium or sulfur.

The intensities of the reflections were estimated using standard multiple film techniques. An intensity scale was prepared from timed oscillations of the (400) reflection. 
V1sual comparison of each reflection with the intensity scale allowed assignment of an uncorrected, relative intensity, $I_{\text {rel }}$, for most of the observed reflections. For each of the Welssenberg layers, medium and strong reflections were read on all four films in order to determine a film absorption factor which could then be used to assign values of $I_{\text {rel }}$ for the strong reflections too dark to be accurately estimated on the first film. Using this procedure, values of $I_{\text {rel }}$ were assigned for the 535 reflections observed in the Weissenberg films.

Comparison of the intensity data for the hk0 and hk2 reflections showed that after correction for angle dependent effects $I_{\text {rel }}(h k 0) \approx I_{\text {rel }}($ hk2). This implied that the atoms of the $\mathrm{Ti}_{2} \mathrm{~S}$ structure occur in two layers perpendicular to the c-axis and confirmed the choice of the $4(\mathrm{~g})$ atom positions Indicated above.

For each reflection the magnitude of the relative structure factor, $\left|F_{\text {rel }}\right|$, was calculated using the formula,

$$
\left|F_{r e l}\right| \propto\left[K / L_{P} \cdot S \cdot A \cdot I_{r e l}\right]^{1 / 2}
$$

The $\mathrm{K} / \mathrm{L}_{\mathrm{P}}$ term corresponds to the Lorentz-polarization correction and the correction required by changes in reflection spot size and shape. These corrections were estimated graphically from the International Tables (17). S corresponds to a linear correction factor whlch was applied to account for 
the splitting of the $\mathrm{K}_{\alpha_{1}}$ and $\mathrm{K}_{\alpha_{2}}$ X-ray radiation components for certain reflections. The absorption correction term $A$ in equation (12) was not used due to the small size of the crystal used for data collection. The values of $\left|F_{\text {rel }}\right|$ calculated in this way were used in the subsequent structure determination.

\section{Structure determination}

The Patterson techniques described above are particularly well-sulted for application to a structure like $\mathrm{TI}_{2} \mathrm{~S}$ where the atom positions occur in two parallel planes. Patterson maps were calculated for varlous layers perpendicular to the c-axis of the unit cell and contained maxima only in the layers $c=0$ and $c=1 / 2$. The resulting two dimensional maps were relatively complex. In order to limit the number of Patterson peaks used in the starting set for superposition maps and to Increase the probability that a chosen peak would correspond to an actual atom position, the Harker (18) sections for the $4(\mathrm{~g})$ atom positions of the Pnnm space group were used.

Harker first showed that the relationship between a particular atom in a unit cell and its symmetry equivalent atoms implied certain restrictions on the Patterson map: For example, an atom in the Pnnm space group at $(x, y, 0)$ Implies a second atom at $(\bar{x}, \bar{y}, 0)$, and the Patterson map will contain the vector between them. If an atom at $(x, y, 0)$ in 
the structure appears in the Patterson map at $(u, v, 0)$, then there must be a second peak at $(2 u, 2 v, 0)$ in the Patterson map. The symmetrically equivalent positions $(1 / 2+x, 1 / 2-y, 1 / 2)$ and $(1 / 2-x, 1 / 2+y, 1 / 2)$ Imply the Harker sections $(1 / 2$, $1 / 2-2 v, 1 / 2)$ and $(1 / 2-2 u, 1 / 2,1 / 2)$. The Pnnm symmetry thus provided three tests that could be applied to increase the probability that a Patterson peak corresponded to an atom position in the unit cell.

Patterson superposition maps were made for only those peaks which obeyed the Harker symmetry conditions. The resulting maps gave structural images masked by spurious overlap, but repeated comparisons of the different maps provided the trial structure illustrated in Figure 1. The six fourffold atom positions of the trial structure, represented by the solid circles labeled A through F, were those input into a Fourler synthesis calculation. The lines and clrcles connected by lines in Figure 1 correspond to regions in the superposition maps where the apparent overlap of peaks occurred in a general, less specific region than the overlap of the six atom positions of the trial structure.

For comparison, Figure 1 also 1liustrates the refined atom positions of the $\mathrm{TI}_{2} \mathrm{~S}$ structure as represented by $\mathrm{TI} 1$. through $S 3$. The agreement between the $s 1 x$ trial atom positions and the refined atom positions can only be described as falr. It is interesting that the recurring areas of general overlap in the superposition teohnique both 


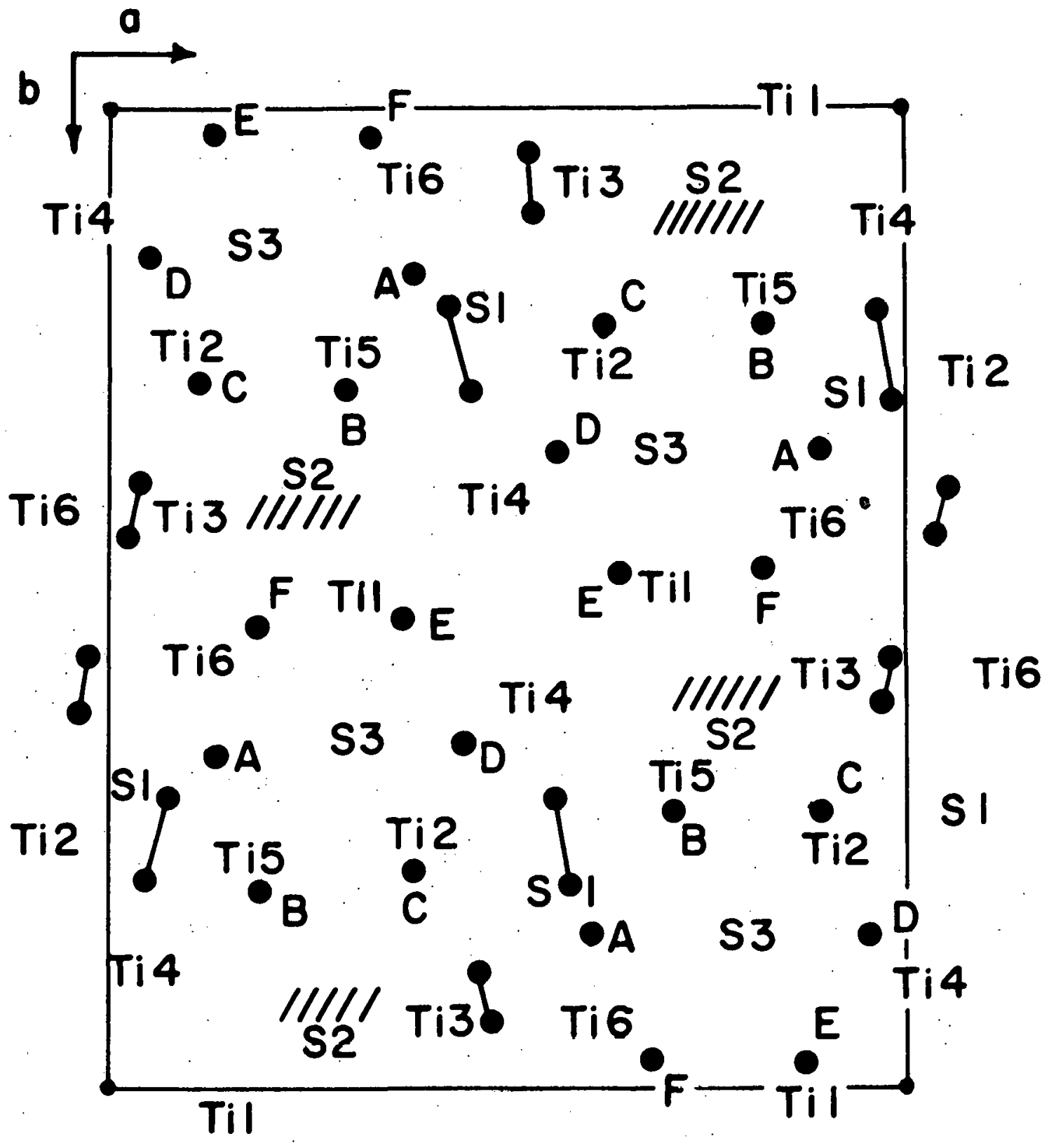

Circles with letters represent well-defined atioms from superposition techniques. Iines correspond to the less well-defined atom positions from superposition tcchniques. The refined atom positions are represented as $\mathrm{T} 1$ and $\mathrm{S}$ atoms.

Figure 1. Comparison of $\mathrm{TI}_{2} \mathrm{~S}$ atom positions from superposition techniques with the refined atom positions 
corresponded closely to actual atom positions in the refined structure. Although only six of the final nine atom positions were input in the Fourler synthesis, the calculation generated the three missing atom positions and provided the correct structure.

4. Structure refinement and description

At this point in the development of the solution to the structure of $\mathrm{TI}_{2} \mathrm{~S}$, the author entered the military service, and the subsequent refinement of the structure was accomplished by Conard (1). The description and discussion of the $\mathrm{Ti}_{2} \mathrm{~S}$ structure is presented in Part II of this thesis.

$$
\text { D. Structure Solution of } \mathrm{Ti}_{8} \mathrm{~S}_{3}
$$

1. Preparation and data collection

$\mathrm{TI}_{8} \mathrm{~S}_{3}$ was first prepared by heating at $1125^{\circ} \mathrm{C}$ a $150 \mathrm{mg}$ pelletized mixture of TiS and titanium metal with an overall Ti/S ratio of 3.0. The partially melted pellet appeared metallic and was quite brittle. A small portion of the pellet was chipped from the bulk sample and a Debye-Scherrer diffraction pattern taken. The extreme complexity of the powder film Indicated the presence of a new phase. Microscopic examination of the sample showed the presence of well-formed, small, needle-like crystals.

Rotation, zero-layer, and first-layer We1ssenberg films Indicated that the crystals corresponded to a $\mathrm{C}$-centered 
monoclinic space group with the crystal rotation axis colncident with the unlque crystallographic b-axis of the unft cell. The approximate lattice parameters calculated from these films were:

$$
\begin{aligned}
& a=32.69(1) \AA \\
& b=3.327(2) \AA \\
& c=19.36(2) \AA \\
& B=139.9(5)^{\circ}
\end{aligned}
$$

The conditions for reflection were:

$$
\begin{array}{ll}
\text { hkl: } & h+k=2 n \\
\text { hol: } & h=2 n \\
\text { Oko: } & k=2 n
\end{array}
$$

$\mathrm{C} 2, \mathrm{Cm}$, and $\mathrm{C} 2 / \mathrm{m}$ were the only space groups consistent with these observations.

Single crystal X-ray diffraction data were collected with a Hilger-Watts four-circle automated diffractometer coupled with an SDS 910 computer, as described by Dahm, Bensen, Nimrod, Fitzwater, and Jacobson (19). Peak-he1ght intensities were measured. Zirconium-filtered Mo $\mathrm{K}_{\alpha}$ radiation was used to obtain data for nonextingulshed reflections in the first two octants with $\theta \leq 30^{\circ}$. Lorentz and polarization corrections were applied, but owing to the small crystal size $(10 \mu \times 10 \mu \times 50 \mu)$, no absorption correction was made. The fluctuation level of the counter was assumed to be proportional to the square root of the total counts, and the 
statistical uncertainties of the intensity data were taken to be $(A+B)^{1 / 2} /(A-B)$, where $A$ and $B$ are peak and background counts, respectively. Of the 2351 intensity data collected, 713 had uncertainties less than $33 \%$.

\section{Structure solution v1a MULTAN}

Early attempts to solve the $\mathrm{Ti}_{8} \mathrm{~S}_{3}$ structure were made utilizing Patterson techniques, Sayre's triple products, and Long's (20) reiterative application of Sayre's triple product relations. Each of these methods was designed to solve a centrosymmetric structure. A Howells, Phillips and Rogers plot (21) proved to be an inconclusive test for a center of symmetry in $\mathrm{T}_{8} \mathrm{~S}_{3}$. It was declded to use MULTAN techniques to attempt the structure determination assuming that the $\mathrm{Ti}_{8} \mathrm{~S}_{3}$ space group was $\mathrm{Cm}$ (noncentrosymmetric).

Normalized structure factors, $E_{\mathfrak{h}}$, were calculated for all of the $\mathrm{Ti}_{8} \mathrm{~S}_{3}$ reflections. The 338 reflections with $\mathrm{E}_{\underset{\sim}{\mathrm{h}}} \geq 1.50$ were used in the MULTAN calculation. For each reflection the SIGMA 2 section of the program calculated all of the phase relationships, equation (6), and the values of $\underset{\sim}{\alpha}(e)$, equation (9). This section of the program also used the $\mathrm{Cm}$ space group symmetry to determine the parity cond1tions necessary for origin definition. The CONVERGE section of the MULTAN program then used the $\alpha_{h}(e)$ values to rank each of the reflections. In a step-by-step process, those reflections with lower values of $\underset{\sim}{\alpha}(e)$ were eliminated. 
The last reflections ellminated were those which gave strong phase relationships and quickly led to multiple phase indications. The six reflections finally remaining included the two necessary for origin definition and four reflections chosen as the starting set for subsequent phase determination. Since the assumed space group was noncentrosymmetric, each of the four reflections of the starting set was assigned a phase corresponding to one of the four values $\pm \pi / 4, \pm 3 \pi / 4$. For each different choice of $\varphi_{\underline{h}}$ for reflections in the starting set, a different set of phases could be calculated.

The phases of the remaining reflections were calculated using the weighted tangent formula,

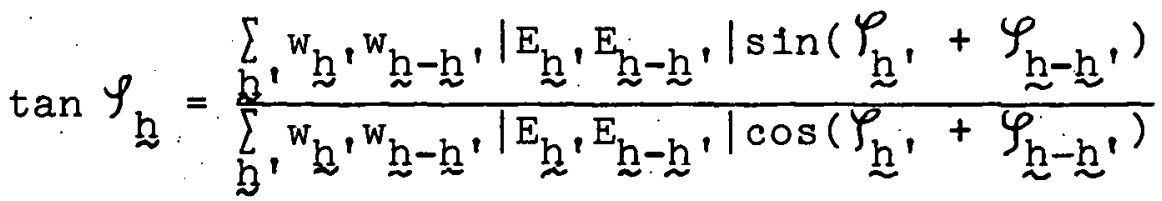

$$
\begin{aligned}
& =\frac{\mathrm{T}_{\mathrm{h}}}{\mathrm{B}_{\mathrm{h}}}
\end{aligned}
$$

where: $\quad w_{\underset{h}{h}}=\tan \mathrm{h}\left(1 / 2 \alpha_{\underset{\sim}{h}}\right)$

and

$$
\alpha_{\underset{\sim}{h}}=\left|E_{\underset{n}{h}}\right|\left(\mathrm{T}_{\underline{h}}^{2}+\mathrm{B}_{\underset{\sim}{h}}^{2}\right)^{1 / 2}
$$

Only 33 of the 256 possible solution sets were calculated. MULTAN techniques provided three criteria by which to determine which of these 33 sets of phases had the highest probability of corresponding to the actual structure. of the three different criteria, only the $\psi_{0}$-test (22) was used, since it was designed specifically for space groups with no 
translational symmetry other than C-centering. $\psi_{0}$ should be a minimum for the correct phasing model.

An E-map was calculated for the set of phases which gave the minimum value for $\psi_{0}$. This E-map was devold of spurious peaks and gave 44 well-resolved peaks which corresponded to chemically reasonable interatomic distances for the trial structure. Titanium and sulfur positions were distinguished by examination of the geometrical arrangement of neighboring peaks. The arrangement of peaks in the E-map suggested the presence of a center of symmetry in the structure.

\section{Structure refinement}

The atom positions suggested by the trial structure were refined by least-squares computation (23) and atomic scatter scattering factors by Hansen, Herman, Lea, and Skillman (24) assuming in the refinement the $\mathrm{Cm}$ space group. Isotropic temperature factors were assumed. After five cycles of refinement, the unweighted $R$ index, $R=\Sigma|| F_{0}|-| F_{C}|| / \Sigma\left|F_{0}\right|$, was 0.108 . A test made for a center of symmetry indicated that one was present within the uncertainty of the atom positions. Assuming the space group $\mathrm{C} 2 / \mathrm{m}$, further refinement reduced the unweighted $R$ index to 0.080 .

The final positional parameters and isotropic temperature factors are given in Table 4. Table 5 lists the nearest neighbors and their interatomjc distances for each atom in $\mathrm{Ti}_{8} \mathrm{~S}_{3} \cdot$ The maximum standard deviations of the interatomic 
Table 4. Final atomic parameters for $\mathrm{Ti}_{8} \mathrm{~S}_{3}$. All atoms occupy four-fold positions (i) $x, 0, z$ of space group $\mathrm{C} 2 / \mathrm{m}$

\begin{tabular}{|c|c|c|c|c|}
\hline Atom & $\mathrm{x}$ & $Y$ & $\mathrm{z}$ & $B\left(\AA^{2}\right)$ \\
\hline $\mathrm{T} 1(1)$ & $0.5795(4)$ & 0.0 & $0.5867(7)$ & $1.0(2)$ \\
\hline $\mathrm{T} 1(2)$ & $0.5943(4)$ & 0.0 & $0.7732(7)$ & $1.1(2)$ \\
\hline $\mathrm{T} 1(3)$ & $0.5890(4)$ & 0.0 & $0.0804(7)$ & $1.0(2)$ \\
\hline $\mathrm{T} 1(4)$ & $0.6223(4)$ & 0.0 & $0.3128(7)$ & $1.0(2)$ \\
\hline $\mathrm{T} 1(5)$ & $0.6712(4)$ & 0.0 & $0.0034(7)$ & $0.9(2)$ \\
\hline $\operatorname{Ti}(6)$ & $0.6956(4)$ & 0.0 & $0.6419(7)$ & $1.0(2)$ \\
\hline $\mathrm{T} 1(7)$ & $0.7180(4)$ & 0.0 & $0.8292(7)$ & $0.9(2)$ \\
\hline $\mathrm{T} 1(8)$ & $0.7033(4)$ & 0.0 & $0.2776(7)$ & $0.7(2)$ \\
\hline$T_{1}(9)$ & $0.8001(4)$ & 0.0 & $0.0692(7)$ & $1.2(2)$ \\
\hline $\mathrm{Ti}(10)$ & $0.7658(4)$ & 0.0 & $0.5729(7)$ & $0.9(2)$ \\
\hline $\mathrm{TI}(11)$ & $0.8363(4)$ & 0.0 & $0.5171(7)$ & $1.0(2)$ \\
\hline$T 1(12)$ & $0.8476(4)$ & 0.0 & $0.8980(7)$ & $0.9(2)$ \\
\hline $\mathrm{Ti}(13)$ & $0.9410(4)$ & 0.0 & $0.1633(7)$ & $1.0(2)$ \\
\hline $\mathrm{TI}(14)$ & $0.0105(4)$ & 0.0 & $0.5945(7)$ & $1.0(2)$ \\
\hline $\mathrm{Ti}(15)$ & $0.9904(4)$ & 0.0 & $0.8926(7)$ & $0.9(2)$ \\
\hline $\operatorname{Ti}(16)$ & $0.4485(4)$ & 0.0 & $0.6199(7)$ & $1.1(2)$ \\
\hline$S(1)$ & $0.5719(6)$ & 0.0 & $0.9517(.9)$ & $0.9(2)$ \\
\hline$S(2)$ & $0.7430(6)$ & 0.0 & $0.2061(10)$ & $1.1(2)$ \\
\hline$S(3)$ & $0.8933(6)$ & 0.0 & $0.4711(10)$ & $0.9(2)$ \\
\hline$S(4)$ & $0.8722(6)$ & 0.0 & $0.7755(10)$ & $1.0(2)$ \\
\hline$S(5)$ & $0.8710(6)$ & 0.0 & $0.2704(10)$ & $0.9(2)$ \\
\hline$S(6)$ & $0.9462(6)$ & 0.0 & $0.7152(10)$ & $1.3(3)$ \\
\hline
\end{tabular}


Table 5. The nearest neighbors and their interatomic distances in $\mathrm{TI}_{8} \mathrm{~S}_{3}$. (Deviations for Ti-S and Ti-Ti distances are $0.050 \AA$ and $0.035 \AA$, respectively)

\begin{tabular}{|c|c|c|c|c|c|c|c|}
\hline $\begin{array}{l}\text { Central } \\
\text { Atom }\end{array}$ & $\begin{array}{c}\text { Type of } \\
\text { nelghbors }\end{array}$ & $\begin{array}{c}\text { No: of } \\
\text { neighbors }\end{array}$ & Distances & $\begin{array}{l}\text { Central } \\
\text { Atom }\end{array}$ & $\begin{array}{c}\text { Type of } \\
\text { neighbors }\end{array}$ & $\begin{array}{l}\text { No. of } \\
\text { neighbors }\end{array}$ & Distances \\
\hline $\mathrm{Ti}(1)$ & $\begin{array}{c}\mathrm{S}(5) \\
\mathrm{S}(3) \\
\mathrm{T} 1(14) \\
\mathrm{T} 1(14) \\
\mathrm{T} 1(6) \\
\mathrm{T} 1(7)\end{array}$ & $\begin{array}{l}2 \\
2 \\
2 \\
2 \\
1 \\
1\end{array}$ & $\begin{array}{l}2.486 \\
2.513 \\
2.820 \\
2.901 \\
3.049 \\
3.159\end{array}$ & $\operatorname{Ti}(5)$ & $\begin{array}{l}S(1) \\
S(2) \\
\mathrm{T} 1(9) \\
\mathrm{T} 1(7) \\
\mathrm{T} 1(13) \\
\mathrm{T} 1(12) \\
\mathrm{T} 1(2)\end{array}$ & $\begin{array}{l}1 \\
1 \\
2 \\
2 \\
2 \\
2 \\
1\end{array}$ & $\begin{array}{l}2.560 \\
2.603 \\
2.770 \\
2.905 \\
2.922 \\
2.930 \\
3.010\end{array}$ \\
\hline$T 1(2)$ & $\begin{array}{l}\mathrm{S}(5) \\
\mathrm{T} 1(14) \\
\mathrm{T} 1(13) \\
\mathrm{T} 1(9) \\
\mathrm{T} 1(5) \\
\mathrm{T} 1(16)\end{array}$ & $\begin{array}{l}2 \\
2 \\
2 \\
2 \\
1 \\
1\end{array}$ & $\begin{array}{l}2.497 \\
2.783 \\
2.793 \\
2.803 \\
3.010 \\
3.149\end{array}$ & $\mathrm{~T}_{1}(6)$ & $\begin{array}{l}\mathrm{S}(3) \\
\mathrm{S}(2) \\
\mathrm{T} 1(8) \\
\mathrm{T} 1(11) \\
\mathrm{T} 1(1) \\
\mathrm{T} 1(7)\end{array}$ & $\begin{array}{l}2 \\
2 \\
2 \\
2 \\
1 \\
1\end{array}$ & $\begin{array}{l}2.509 \\
2.533 \\
2.875 \\
2.903 \\
3.049 \\
3.097\end{array}$ \\
\hline $\mathrm{T} 1(3)$ & $\begin{array}{c}S(4) \\
S(1) \\
\operatorname{TI}(15) \\
\mathrm{T} 1(3) \\
\mathrm{T} 1(15) \\
\mathrm{T} 1(8) \\
\mathrm{T} 1(12)\end{array}$ & $\begin{array}{l}2 \\
1 \\
2 \\
1 \\
2 \\
1 \\
2\end{array}$ & $\begin{array}{l}2.454 \\
2.586 \\
2.868 \\
2.879 \\
2.881 \\
2.884 \\
2.960\end{array}$ & $\mathrm{~T} 1(7)$ & $\begin{array}{l}\mathrm{S}(2) \\
\mathrm{S}(5) \\
\mathrm{T} 1(5) \\
\mathrm{T} 1(9) \\
\mathrm{T} 1(6) \\
\mathrm{T} 1(9) \\
\mathrm{T} 1(1)\end{array}$ & $\begin{array}{l}2 \\
2 \\
2 \\
2 \\
1 \\
1 \\
1\end{array}$ & $\begin{array}{l}2.489 \\
2.520 \\
2.905 \\
2.955 \\
3.097 \\
3.124 \\
3.159\end{array}$ \\
\hline $\operatorname{Ti}(4)$ & $\begin{array}{c}\mathrm{S}(4) \\
\mathrm{S}(6) \\
\mathrm{T} 1(11) \\
\mathrm{T} 1(10) \\
\mathrm{T} 1(15) \\
\mathrm{T} 1(8)\end{array}$ & $\begin{array}{l}2 \\
2 \\
2 \\
2 \\
2 \\
1\end{array}$ & $\begin{array}{l}2.488 \\
2.490 \\
2.938 \\
2.943 \\
3.120 \\
3.196\end{array}$ & & & & \\
\hline
\end{tabular}


Table 5. (Continued)

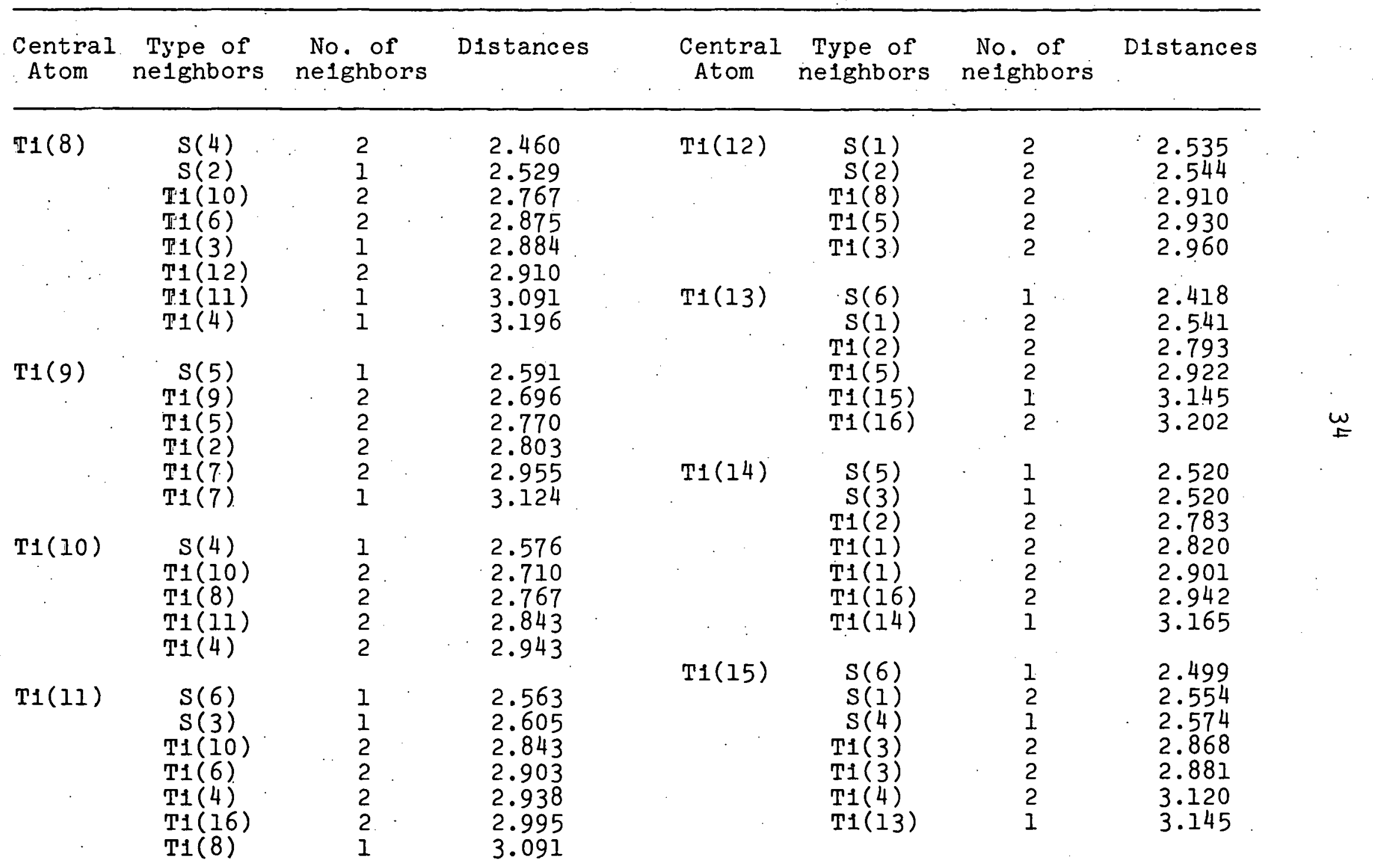


Table 5. (Continued).

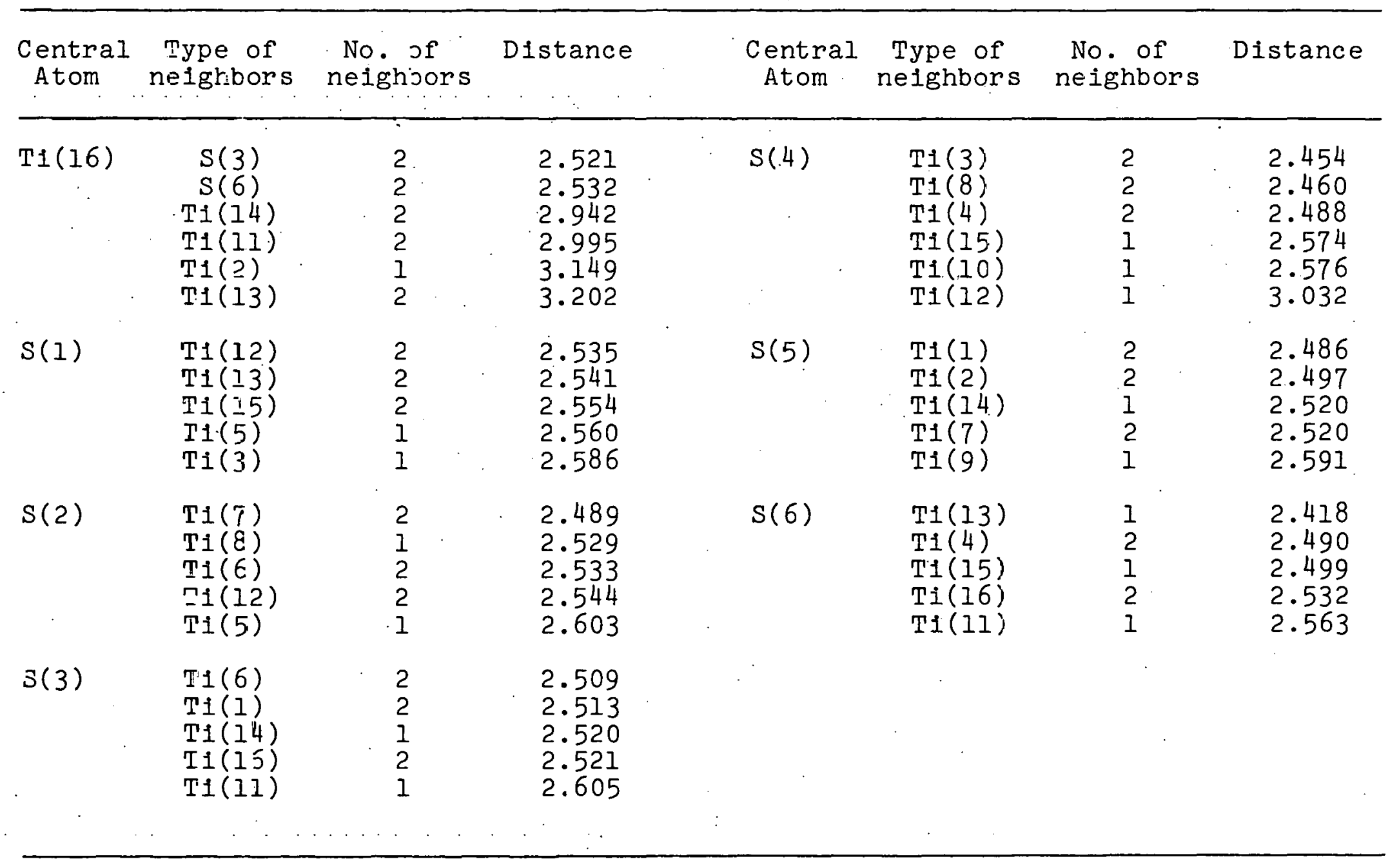


distances were $0.050 \AA$ for $\dot{T} 1-S$ distances and $0.035 \AA$ for T1-T1 distances.

4. Accuracy of the MULTAN technique

The success of the MULTAN techniques in the structure solution of $\mathrm{Ti}_{8} \mathrm{~S}_{3}$ where other attempts had falled is deserving of comment. The failure of Sayre's triple product relations to provide a structure solution (even though $\mathrm{Ti}_{8} \mathrm{~S}_{3}$ was centrosymmetric) may be related to the arbitrary choice of reflections assigned symbols and used in a starting set for further phase determination. When the starting set of reflections chosen by MULTAN were used in Sayre's triple product relations, the phases (signs) for the 70 strongest reflections of $\mathrm{T}_{8} \mathrm{~S}_{3}$, were correctly determined.

The close agreement between the 44 atom positions of the trial structure and the refined atom positions seemed remarkable. Table 6 compares the calculated phases with the final phase values after refinement for a random selection of reflections and 1llustrates hielr close agreement. This close agreement is true for all of the reflections. The accuracy of the calculated phase values may be the reason for both the lack of spurious peaks in the E-map and the accuracy of the trial structure. In the derivation of the formulas used in the MULTAN calculation, an assumption was made that the structure is composed only of equal atoms (10). The small difference between titanium and sulfur of six electrons 
Table 6. Comparison of phase values calculated by MULTAN techniques, with the refined phase values for $\mathrm{TI}_{8} \mathrm{~S}_{3}$. (Phase in degrees)

\begin{tabular}{|c|c|c|c|c|c|}
\hline \multicolumn{3}{|c|}{ Reflection } & \multirow{2}{*}{$\begin{array}{l}\begin{array}{l}\text { Refined } \\
\text { Phase }\end{array} \\
0\end{array}$} & \multirow{2}{*}{$\begin{array}{l}\text { MULTAN } \\
\text { Phase } \\
360.2\end{array}$} & \multirow{2}{*}{$\begin{array}{c}\text { Difference } \\
\text { (Absolute Value) }\end{array}$} \\
\hline 8 & 2 & $\dot{\overline{9}}$ & & & \\
\hline 20 & 2 & 4 & 180 & 164.4 & 15.6 \\
\hline 1 & 3 & 3 & 180 & 170.4 & 9.6 \\
\hline 10 & 2 & 10 & 180 & 186.6 & 6.6 \\
\hline 7 & 3 & $\bar{\sigma}$ & 180 & 183.6 & 3.6 \\
\hline 42 & 0 & 15 & 180 & 185.0 & 5.0 \\
\hline 28 & 0 & 0 & 180 & 185.5 & 5.5 \\
\hline 29 & 1 & $\overline{16}$ & 0 & 4.8 & 4.8 \\
\hline 8 & 0 & 2 & 180 & 216.0 & 36.0 \\
\hline 6 & 0 & 10 & 0 & 2.2 & 2.2 \\
\hline 16 & 2 & 0 & 0 & 351.4 & 8.6 \\
\hline 38 & 2 & 13 & 180 & 185.1 & 5.1 \\
\hline 6 & 0 & 2 & 0 & 348.9 & 11.1 \\
\hline 8 & 2 & $\overline{19}$ & 0 & 338.4 & 21.6 \\
\hline 4 & 2 & 5 & 0 & 354.2 & 5.8 \\
\hline 5 & 1 & 2 & 0 & 6.8 & 6.8 \\
\hline 30 & 0 & $\overline{26}$ & 0 & 26.2 & 26.2 \\
\hline 0 & 0 & 16 & 180 & 195.8 & 15.8 \\
\hline 27 & 1 & $\overline{2 I}$ & 0 & 358.8 & 1.2 \\
\hline 17 & 3 & 3 & 180 & 184.0 & 4.0 \\
\hline 33 & 1 & $\overline{19}$ & 0 & 359.6 & 0.4 \\
\hline 8 & 0 & 1 & 180 & 190.9 & 10.9 \\
\hline
\end{tabular}


provided a case where the equal atom approximation was closely approached, perhaps explaining the accuracy of the calculated phase determination. 
PART II. STRUCTURAL CORRELATIONS AND CHEMICAL BONDING 


\section{INTRODUCTION}

The successful structural solution of the $\mathrm{TI}_{2} \mathrm{~S}$ and $\mathrm{TI}_{8} \mathrm{~S}_{3}$ structure types led naturally to a comparison of their structural similarities and differences. The structural features of these two phases are features common to a large number of other structure types. A large portion of this work 1s devoted to a detailed comparison of the features common to a large number of structure types with features similar to those of $\mathrm{TI}_{2} \mathrm{~S}$ and $\mathrm{TI}_{8} \mathrm{~S}_{3}$.

In studying the structural similarities and differences for the structure types under discussion here, a continuing attempt was made to understand the structural features in terms of qualitative bonding models. The complexity of the structures discussed here precluded any type of rigorous theoretical treatment (e.g., band structure approach). Rather, the approach used throughout this study was to observe the structural similarities and differences and then to continualiy question the reasons why the similarities and differences occur.

The recurring geometrical features found in these structure types, 1 .e.., trigonal prismatic nonmetal cooralnation and the metal coordination units, suggest that the structural features may be best understood in terms of a hybrid orbital model. Although it is certainly true that not all of the structural features can be understood within the 
framework of a simple hybrid orbital model, such a model seemed to be fruitful approach in describing the sigmabonding contribution of the individual atoms making up the structures of the phases described here. It was felt that such an approach also allowed a framework within which a better understanding of the structural similarities and differences for the structure types under discussion was possible.

The basic weakness of this type of approach is that any specific consideration of the possible $\pi$-bonding interactions between atoms is ignored. It is the point of view adopted here that the structure features under study depend primarily on the sigma-bonding contribution of the atoms involved. For comparison, the benzene molecular structure might be considered in terms of the $\mathrm{sp}^{2}$ hybrid orbital combination bonding contribution of each carbon atom. It is this bonding contribution which, in effect, determines the molecular geometry. Obviously, the $\pi$-bonding contribution is important in understanding the chemical properties of benzene, but $1 t$ is felt that the inclusion of $\pi$-bonding interaction is not necessary in describing the basic molecular structure. 
II. THE TI $I_{2} S$ AND $\mathrm{TI}_{8} \mathrm{~S}_{3}$ STRUCTURES

\section{A. Introduction}

In Chapter II the methods used to solve the structure of $\mathrm{Ti}_{2} \mathrm{~S}$ and $\mathrm{TI}_{8} \mathrm{~S}_{3}$ were described. In the following description of the $\mathrm{Ti}_{2} \mathrm{~S}$ and $\mathrm{Ti}_{8} \mathrm{~S}_{3}$ structures, particular emphasis will be placed on the discussion of those structural features which are common to a large number of other structure types, for the $\mathrm{TI}_{2} \mathrm{~S}$ and $\mathrm{Ti}_{8} \mathrm{~S}_{3}$ structures are two of a variety of structures that form a structural class consisting of structures assumed by a large number of transition metal chalcogenide and pnictide phases. A major theme of this thesis will be that an analysis of the structural-chemical principles underlying the recurrent features in this class of compounds leads to information about the nature of the chemical interactions in the compounds in general, and in $v$ $\mathrm{TI}_{2} \mathrm{~S}$ and $\mathrm{TI}_{8} \mathrm{~S}_{3}$ in particular.

\section{B. Structure Descriptions}

The structure of $\mathrm{TI}_{2} \mathrm{~S}$, which has already been described by Conard ( 1 ) in his thesis, is illustrated in Figures 2 and 3. The system used to reprcaent the atoms in H'igure 3 will be used throughout for depleting the structures of other phases of this structural class. Nonmetal atom positions w1ll be represented by the symbols $x$ or $\otimes$ where the absence or presence of the circle is used to distinguish the nonmetal 


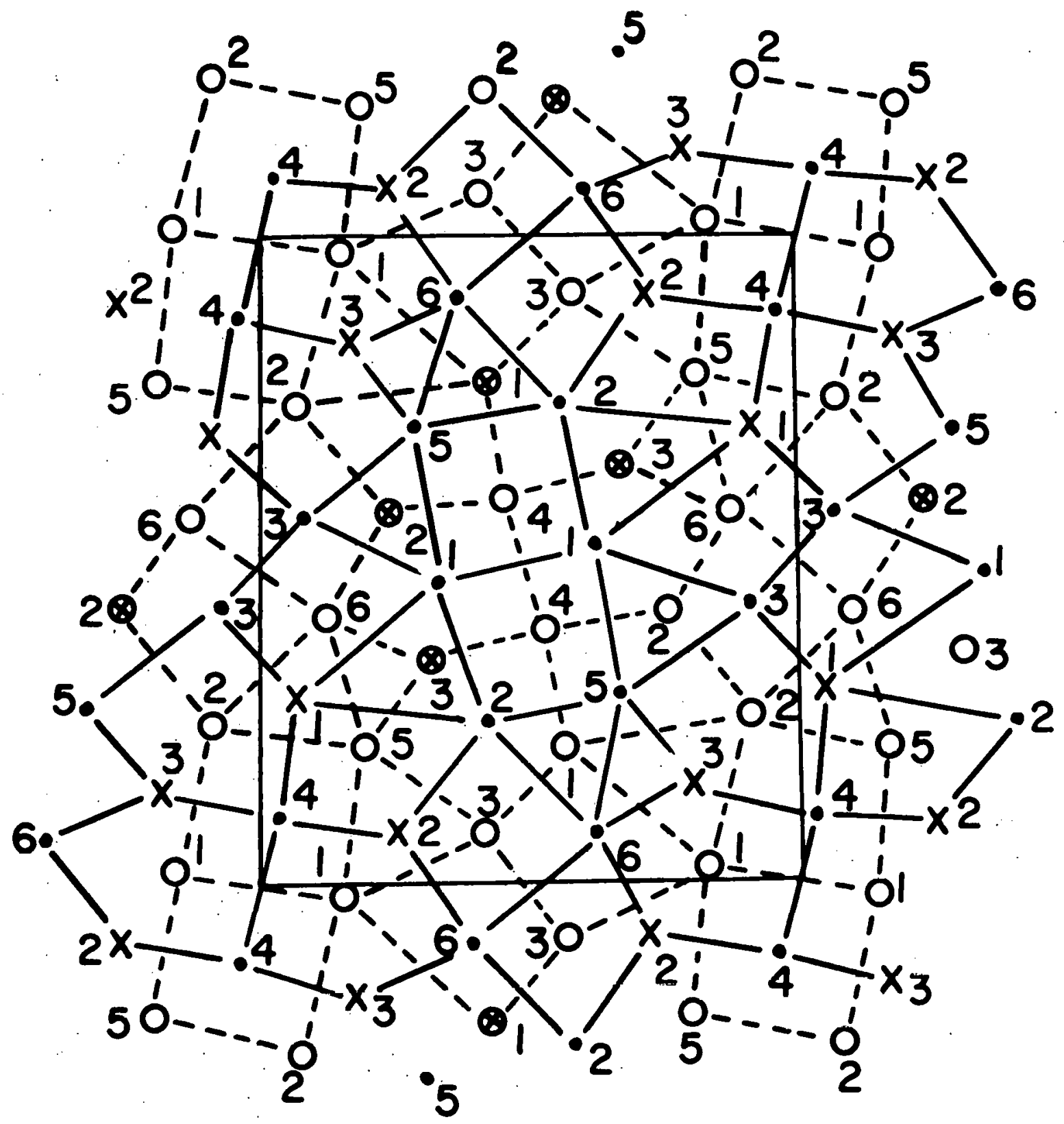

Solid lines represent the coordination polyhedra of atom positions in the layer at $a=1 / 2$. Broken Iines represent the polyhedra of atom positions in the layer at $z=0$.

Figure 2. The $\mathrm{Ti}_{2} \mathrm{~S}$ structure 


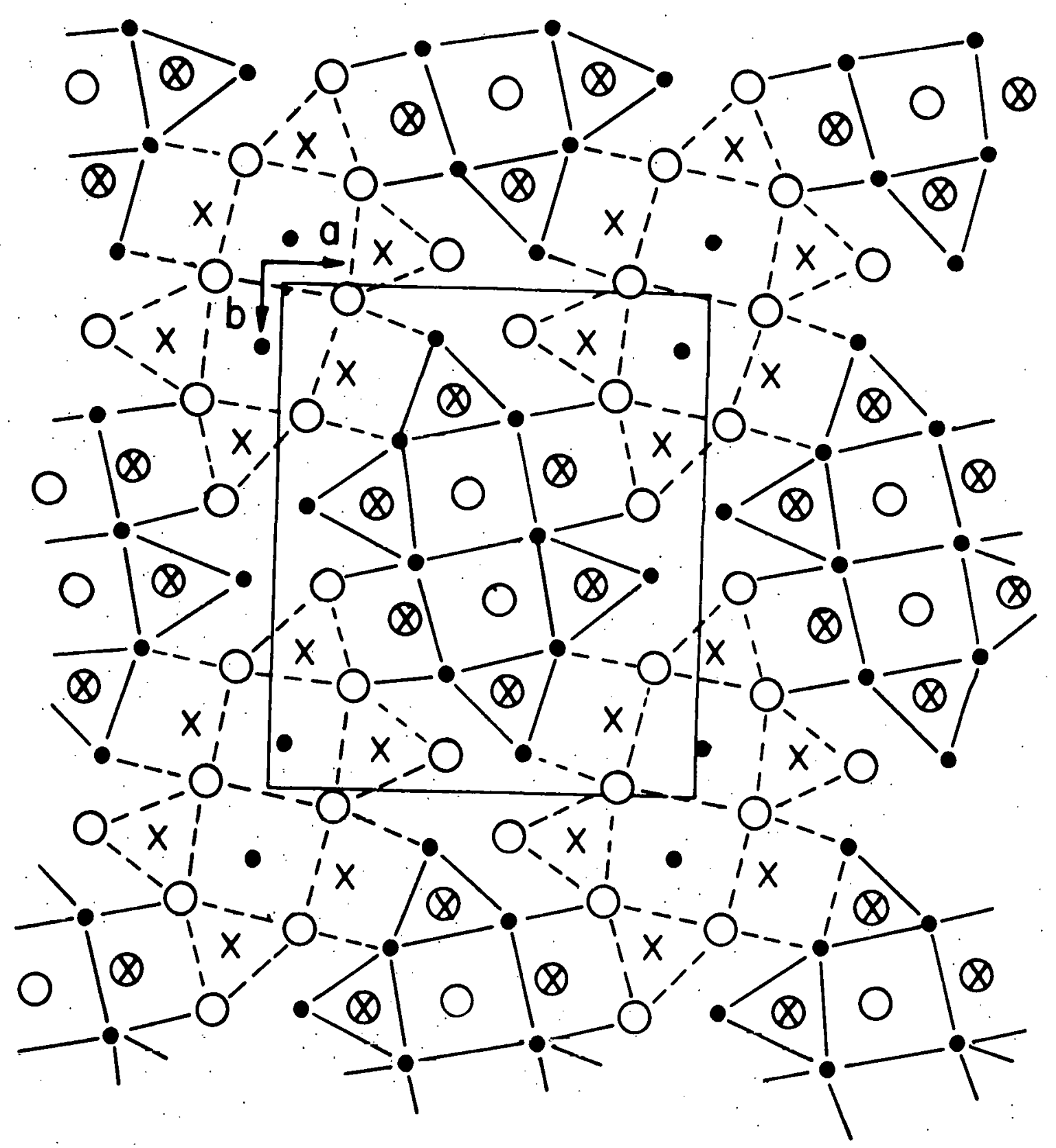

$x$ and $\otimes$ correspond to nonmetal atom positions while and o represent metal atom positions in the two distinct layers of the structure: $x$ and represent atoms in the same layer while $\otimes$ and 0 represent atom positions in the same, but distinct, layer. The same symbollsm will be used to represent other structures below.

Figure 3. The $\mathrm{T}_{2} \mathrm{~S}$ structure as the packing of titanium cubes and sulfur trigonal prisms 
atom positions which occur in the two separate layers which characterize the structures. Similarly, the symbols $\bullet$ and 0 represent metal atom positions in the two distinct layers. The symbols with clrcles, whether metal or nonmetal, represent atom positions in the same layer.

The solid and dashed lines. in Figure 2 illustrate the partial coordination polyhedra of titanium and sulfur atoms. Solid lines represent the partial coordination polyhedra (P.C.P.) about atoms centered at $z=1 / 2$, while the dashed lines correspond to the P.C.P. for atoms centered at $z=0$. A drawing of this type (Figure 2) emphasizes the partial coordination polyhedra present in the structure and illustrates the face, edge, and corner sharing of the various polyhedra. The structure of $\mathrm{Ti}_{2} \mathrm{~S}$ lllustrates only one of the large number of possible ways that the observed P.C.P. are able to pack in forming a solid structure.

Figure 4 illustrates the $\mathrm{Ti}_{8} \mathrm{~S}_{3}$ structure, emphasizing the different types of metal coordination polyhedra present.. The first impression upon viewing this structure is one of the extreme complexity of this phase which is indicated by the presence of 88 atoms in a unit cell with axes 32.69(1) and 19.36(2) . The $\mathrm{T1}_{8} \mathrm{~S}_{3}$ structure 11lustrates a second way in which the coordination polyhedra of iltanium and sulfur can pack to form a stable phase.

$\mathrm{Ti}_{2} \mathrm{~S}$ and $\mathrm{TI}_{8} \mathrm{~S}_{3}$ have a large number of common structural features which can be seen by comparing Figures 2 and 4 . 


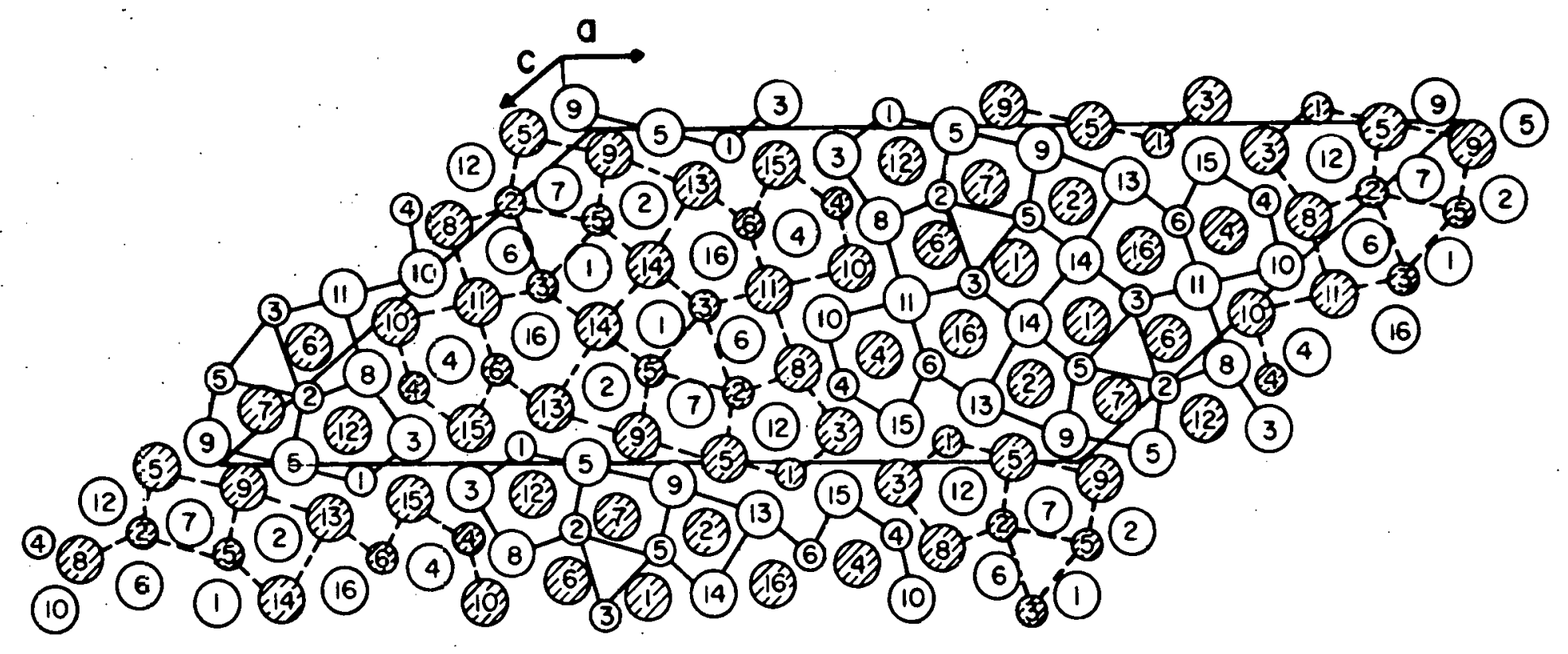

The solid and broken lines illustrate the metal coordination polyhedra. Large circles represent titanium atom positions while small circles represent sulfur atom positions. Shaded and nonshaded circles are atom positions located in the two distinct layers.

Figure 4. The $\mathrm{TI}_{8} \mathrm{~S}_{3}$ structure 
Both structures are 1llustrated as projections of the atomic positions along the short crystallographic axis of each unit cell. All of the atom positions occur in one of two planes perpendicular to the short axis. Each plane of atom positions lies on a mirror plane, which is a symmetry element common to both the Pnnm space group of $\mathrm{T}_{2} \mathrm{~S}$ and the $\mathrm{C} 2 / \mathrm{m}$ space group of $\mathrm{Ti}_{8} \mathrm{~S}_{3}$. Nonmetal atoms of both phases have capped-trigonal prismatic coordination polyhedra where the 3-fold axis of the trigonal prisms are elther perpendicular to the plane of projection, as for $S(1)$ and $S(2)$ of both phases, or parallel to the plane of projection, as for $S(3)$ of $\mathrm{TI}_{2} \mathrm{~S}$ and $\mathrm{S}(6)$ of $\mathrm{TI}_{8} \mathrm{~S}_{3}$. For the two structures the metal coordination polyhedra are very similar and are characterized by high coordination numbers for titanium atoms. The atoms of both phases are well-packed in the sense that neither the $\mathrm{TI}_{2} \mathrm{~S}$ nor $\mathrm{TI}_{8} \mathrm{~S}_{3}$ structure contains any region where an additional titanium or sulfur atom would fit without implying interatomic distances substantially shorter than what would be expected from chemical considerations.

Tables 5 and 7 list the interatomic distances for $\mathrm{TI}_{8} \mathrm{~S}_{3}$ and $\mathrm{TI}_{2} \mathrm{~S}$, respectively. Reference to these tables shows a marked similarity in metal-metal and metal-nonmetal distances for the two phases. The similarity in interatomic distances is even more pronounced if one compares only the 
Table 7. Interatomic distances for $\mathrm{TI}_{2} \mathrm{~S}$. All distances (TI-T1) less than $3.30 \AA$ are listed

\begin{tabular}{|c|c|c|c|}
\hline $\begin{array}{l}\text { Central } \\
\text { Atom }\end{array}$ & $\begin{array}{l}\text { Ne1ghbor } \\
\text { Atom }\end{array}$ & $\begin{array}{l}\text { Interatomic } \\
\text { Distance }(\AA)\end{array}$ & $\begin{array}{c}\text { Number of } \\
\text { Bonds }\end{array}$ \\
\hline $\mathrm{T} 1(1)$ & $\begin{array}{r}\mathrm{T} 1(6) \\
\mathrm{S}(3) \\
\mathrm{T} 1(4) \\
\mathrm{T} 1(4) \\
\mathrm{S}(2) \\
\mathrm{T} 1(5)\end{array}$ & $\begin{array}{l}3.154 \\
2.488 \\
2.843 \\
2.952 \\
2.525 \\
3.241\end{array}$ & $\begin{array}{l}2 \\
2 \\
2 \\
2 \\
2 \\
1\end{array}$ \\
\hline$T 1(2)$. & $\begin{array}{r}\mathrm{T} 1(4) \\
\mathrm{S}(3) \\
\mathrm{T} 1(5) \\
\mathrm{T} 1(3) \\
\mathrm{S}(1) \\
\mathrm{S}(2) \\
\mathrm{T} 1(5)\end{array}$ & $\begin{array}{l}2.953 \\
2.482 \\
3.049 \\
2.853 \\
2.474 \\
2.848\end{array}$ & $\begin{array}{l}2 \\
2 \\
2 \\
2 \\
2 \\
1\end{array}$ \\
\hline$T 1(3)$ & $\begin{array}{r}\mathrm{S}(2) \\
\mathrm{T} 1(2) \\
\mathrm{T} 1(6) \\
\mathrm{T} 1(6) \\
\mathrm{S}(1) \\
\mathrm{T} 1(3) \\
\mathrm{T} 1(5) \\
\mathrm{T} 1(1)\end{array}$ & $\begin{array}{l}2.438 \\
2.853 \\
2.793 \\
2.890 \\
2.516 \\
3.004 \\
3.071 \\
3.246\end{array}$ & $\begin{array}{l}2 \\
2 \\
2 \\
2 \\
1 \\
1 \\
1 \\
1\end{array}$ \\
\hline $\mathrm{Ti}(4)$ & $\begin{array}{r}\mathrm{T} 1(1) \\
\mathrm{T} 1(1) \\
\mathrm{T} 1(2) \\
\mathrm{T} 1(5) \\
\mathrm{S}(3) \\
\mathrm{S}(2) \\
\mathrm{S}(1) \\
\mathrm{T} 1(4)\end{array}$ & $\begin{array}{l}2.843 \\
2.952 \\
2.953 \\
2.838 \\
2.501 \\
2.567 \\
2.616 \\
3.158\end{array}$ & $\begin{array}{l}2 \\
2 \\
2 \\
2 \\
1 \\
1 \\
1 \\
1\end{array}$ \\
\hline $\mathrm{Ti}(5)$ & $\begin{array}{r}\mathrm{S}(1) \\
\mathrm{T} 1(4) \\
\mathrm{S}(2) \\
\mathrm{T} 1(2) \\
\mathrm{S}(3) \\
\mathrm{T} 1(3) \\
\mathrm{T} 1(6) \\
\mathrm{T} 1(2) \\
\mathrm{T} 1(1)\end{array}$ & $\begin{array}{l}2.490 \\
2.838 \\
2.472 \\
3.049 \\
2.437 \\
3.071 \\
3.123 \\
3.241 \\
3.241\end{array}$ & $\begin{array}{l}2 \\
2 \\
2 \\
2 \\
1 \\
1 \\
1 \\
1 \\
1\end{array}$ \\
\hline
\end{tabular}


Table 7... (Continued)

\begin{tabular}{|c|c|c|c|}
\hline $\begin{array}{l}\text { Central } \\
\text { Atom }\end{array}$ & $\begin{array}{l}\text { Neighbor } \\
\text { Atom }\end{array}$ & $\begin{array}{l}\text { Interatomic } \\
\text { Distance ( }(\AA)\end{array}$ & $\begin{array}{l}\text { Number of } \\
\text { Bonds }\end{array}$ \\
\hline $\mathrm{T} 1(6)$ & $\begin{array}{r}S(1) \\
\mathrm{T} 1(3) \\
\mathrm{T} 1(3) \\
\mathrm{T} 1(1) \\
\mathrm{S}(3) \\
\mathrm{S}(2) \\
\mathrm{T} 1(5)\end{array}$ & $\begin{array}{l}2.528 \\
2.793 \\
2.890 \\
3.154 \\
2.442 \\
2.724 \\
3.123\end{array}$ & $\begin{array}{l}2 \\
2 \\
2 \\
2 \\
1 \\
1 \\
1\end{array}$ \\
\hline$S(I)$ & $\begin{array}{l}\operatorname{T1}(2) \\
\operatorname{T1}(6) \\
\operatorname{T1}(5) \\
\operatorname{T1}(3) \\
\operatorname{T1}(4)\end{array}$ & $\begin{array}{l}2.474 \\
2.528 \\
2.490 \\
2.516 \\
2.616\end{array}$ & $\begin{array}{l}2 \\
2 \\
2 \\
1 \\
1\end{array}$ \\
\hline$S(2)$ & $\begin{array}{l}\operatorname{T1}(1) \\
\operatorname{T1}(5) \\
\operatorname{T1}(3) \\
\operatorname{T1}(4) \\
\operatorname{T1}(6) \\
\operatorname{T1}(2)\end{array}$ & $\begin{array}{l}2.525 \\
2.472 \\
2.438 \\
2.567 \\
2.724 \\
2.848\end{array}$ & $\begin{array}{l}2 \\
2 \\
2 \\
1 \\
1 \\
1\end{array}$ \\
\hline$s(3)$ & $\begin{array}{l}\operatorname{Ti}(1) \\
\operatorname{TI}(2) \\
\operatorname{TI}(5) \\
\operatorname{TI}(6) \\
\operatorname{TI}(4)\end{array}$ & $\begin{array}{l}2.488 \\
2.482 \\
2.437 \\
2.442 \\
2.501\end{array}$ & $\begin{array}{l}2 \\
2 \\
1 \\
1 \\
1\end{array}$ \\
\hline & & . . & \\
\hline
\end{tabular}


distances for those coordination polyhedra which are common to both $\mathrm{TI}_{2} \mathrm{~S}$ and $\mathrm{Ti}_{8} \mathrm{~S}_{3}$.

The general structural features discussed above which are common to $\mathrm{TI}_{2} \mathrm{~S}$ and $\mathrm{Ti}_{8} \mathrm{~S}_{3}$ (capped trigonal prismatic coordination of sulfur and atom positions in mirror planes) also occur for a large number of other transition metal chalcogenide and pnictide phases. The recurrence of similar structural features for a varlety of different phases indicates that $\mathrm{TI}_{2} \mathrm{~S}$ and $\mathrm{Ti}_{8} \mathrm{~S}_{3}$ are only two members of a larger structural class. 
III. STRUCTURAL CLASS

\section{A. Introduction}

In the context of this discussion a structural class is defined as a set of structures with a number of common structural features which distinguish them from other phases formed with similar stolchiometry and from similar chemical components. A considerable portion of this chapter will be devoted to a detailed description of the structural features which define this class of compounds. In this and the following chapters the structural features, their frequency of occurrence, their correlation with stolchiometry and metallic element, etc., will provide the basis and limitations for qualitative bonding models that will be devised to help explain the structures of this class of compounds.

\section{B. Structures Forming this Structural Class}

The particular compounds with structures that belong to the structural class under consideration are the binary transition metal chalcogenides and pnictides. In particular, the nonmetal components of the known compounds with structures in the class are sulfur, selenium, phosphorous or arsenic. The metal-to-nonmetal ratios $(\mathrm{Me} / \mathrm{X}$ ) of the typically stolchiometric phases with structures in this class fall in the range given by $1.0<\mathrm{Me} / \mathrm{X}<3.0$. For the phases formed from transition elements and the nonmetals discussed above 
with $M e / X \leq 1.0$, the structures contain separate metal and nonmetal layers, while the structures of this class are characterized by the presence of both metal and nonmetal atoms within the same layer. The structural class under consideration here will be referred to as a layered class, but it is important to note that the layering is not of the Me-X-Me-X... variety found for the more nonmetal-rich compounds.

Furthermore, the structures formed by the transition metal chalcogenides and pnictides under consideration where $\mathrm{Me} / \mathrm{X} \geq 3.0, \underline{1} \cdot \underline{e}_{.}$, the more metal-rich phases (e.g., $\mathrm{Ta}_{6} \mathrm{~S}, \alpha-$ and $\beta-V_{3} S$ and $Z r_{9} S_{2}$ ) do not contaln the unique, short crystallographic axis common to the layered class. In addition, the predominant metal coordination polyhedra for those phases where $\mathrm{Me} / \mathrm{X} \geq 3.0$ can be best described in terms of Kasper polyhedra (25).

Transition metal atoms that form the phases of the layered class correspond to all three transition series except for the Sc, $\mathrm{Cu}$ and $\mathrm{Zn}$ families. Phases formed from metals in these families have structural features quite different than those of this layered class. Of the phases with structures that are members of this layered class, the majority contain metal atoms from the group IV and VB transition metals.

Table 8 lists representative compounds with structures that are members of the iayered structural class, an identification of the structure-type, space group, laltice 
Table. 8. Structural class

\begin{tabular}{|c|c|c|c|c|c|c|c|c|c|}
\hline \multirow{3}{*}{$\begin{array}{l}\text { Structure } \\
\text { Type }\end{array}$} & \multirow[b]{3}{*}{ Phase } & \multicolumn{6}{|c|}{ Lattice Parameters } & \multirow{3}{*}{$\begin{array}{l}\text { Space } \\
\text { Group }\end{array}$} & \multirow{3}{*}{ References } \\
\hline & & An & gstrom Un & its & & Degrees & & & \\
\hline & & a & $\mathrm{b}$ & c & $\alpha$ & $\beta$ & $\gamma$ & & \\
\hline \multirow{7}{*}{$\mathrm{Ta}_{2} \mathrm{P}$} & $\mathrm{Ta}_{2} \mathrm{P}$ & 14.419 & 11.552 & 3.399 & 90 & 90 & 90 & Pnnm & 26 \\
\hline & $\mathrm{TI}{ }_{2} \mathrm{~S}$ & 11.367 & 14.060 & 3.326 & 90 & 90 & 90 & Pnnm & 1,27 \\
\hline & $\mathrm{TI}_{2} \mathrm{Se}$ & 11.77 & 14.57 & 3.515 & 90 & 90 & 90 & Pnnm. & 28 \\
\hline & $\mathrm{Hf}_{2} \mathrm{P}$ & 1.5 .031 & 12.258 & 3.5738 & 90 & 90 & 90 & Pnnm & 29 \\
\hline & $\mathrm{Ta}_{2} \mathrm{As}$ & 14.7680 & 11.8373 & 3.4696 & 90 & 90 & 90 & Pnnm & 30 \\
\hline & $\mathrm{Zr}_{2} \mathrm{~S}$ & 12.46 & 14.95 & $3 \cdot 33$ & 90 & 90 & 90 & Pnnm & 28 \\
\hline & $\mathrm{Zr}_{2} \mathrm{Se}$ & 12.675 & 15.78 & 3.61 & 90 & 90 & 90 & Pnnm & 28 \\
\hline $\mathrm{Ti}_{8}$ & $\mathrm{Ti}_{8} \mathrm{~S}_{3}$ & 32.69 & 3.327 & 19.36 & 90 & 139.9 & 90 & $\mathrm{C} 2 / \mathrm{m}$ & This work \\
\hline $\mathrm{Nb}_{21} \mathrm{~S}_{8}$ & $\mathrm{Nb}_{21} \mathrm{~S}_{8}$ & 16.794 & 16.794 & .3 .359 & 90 & 90 & 90 & $\mathrm{I} 4 / \mathrm{m}$ & 32 \\
\hline $\mathrm{Nb}_{14} \mathrm{~S}_{5}$ & $\mathrm{Nb}_{14} \mathrm{~S}_{5}$ & 18.430 & 3.374 & 19.797 & 90 & 90 & 90 & Pnma & 33 \\
\hline \multirow{2}{*}{$\mathrm{Ti}_{5} \mathrm{Te}_{4}$} & $\mathrm{Ti}_{5} \mathrm{Te}_{4}$ & 10.154 & 10.164 & 3.772 & 90 & 90 & 90 & $\mathrm{I} 4 / \mathrm{m}$ & 34 \\
\hline & $\mathrm{Nb}_{5} \mathrm{Se}_{4}$ & 9.871 & 9.871 & 3.4529 & 90 & 90 & 90 & $\mathrm{I} 4 / \mathrm{m}$ & 35 \\
\hline $\mathrm{Nb}_{2} \mathrm{Se}$ & $\mathrm{Nb}_{2} \mathrm{Se}$ & 13.995 & 3.4298 & 9.306 & 90 & 90.04 & 90 & $\mathrm{C} 2 / \mathrm{m}$ & 36 \\
\hline $\mathrm{Hf}_{3} \mathrm{P}_{2}$ & $\mathrm{Hf}_{3} \mathrm{P}_{2}$ & 10.138 & 3.578 & 9.881 & 90 & 90 & 90 & Pnma & 37 \\
\hline \multirow[t]{2}{*}{$\mathrm{Nb}_{7} \mathrm{P}_{4}$} & $\mathrm{Nb}_{7} \mathrm{P}_{4}$ & 14.950 & 3.440 & 13.848 & 90 & 1.04 .74 & 90 & $\mathrm{C} 2 / \mathrm{m}$ & 38 \\
\hline & $\mathrm{Nb}_{7} \mathrm{As}_{4}$ & 15.3716 & 3.5242 & 14.1920 & 90 & 104.74 & 90 & $\mathrm{C} 2 / \mathrm{m}$ & 30 \\
\hline $\mathrm{Mb}_{5} \mathrm{P}_{3}$ & $\mathrm{Nb}_{5} \mathrm{P}_{3}$ & 25.384 & 3.433 & 11.483 & 90 & 90 & 90 & Pnma & 39 \\
\hline $\mathrm{Nb}_{8} \mathrm{P}_{5}$ & $\mathrm{Nb}_{8} \mathrm{P}_{5}$ & 26.1998 & 9.4652 & 3.4641 & 90 & 90 & 90 & Pbam & 40 \\
\hline
\end{tabular}




\begin{tabular}{llllllllll}
$\mathrm{Mo}_{8} \mathrm{P}_{5}$ & $\mathrm{Mo}_{8} \mathrm{P}_{5}$ & 9.399 & 3.209 & 6.537 & 90 & 109.59 & 90 & $\mathrm{Pm}$ & 41 \\
$\mathrm{~V}_{12} \mathrm{P}_{7}$ & $\mathrm{~V}_{12} \mathrm{P}_{7}$ & 9.299 & 9.299 & 3.2790 & 90 & 90 & 120 & $\mathrm{P} 6_{3} / \mathrm{m}$ & 42 \\
$\mathrm{Fe}_{2} \mathrm{P}$ & $\mathrm{Fe}{ }_{2} \mathrm{P}$ & 5.865 & 5.865 & 3.456 & 90 & 90 & 120 & $\mathrm{P} 62 \mathrm{~m}$ & 43 \\
$\mathrm{Co}_{2} \mathrm{P}$ & $\mathrm{Co}_{2} \mathrm{P}$ & 5.646 & 3.513 & 6.608 & 90 & 90 & 90 & $\mathrm{Pnma}$ & 44 \\
& $\mathrm{Ru}_{2} \mathrm{P}$ & 5.902 & 3.859 & 6.896 & 90 & 90 & 90 & $\mathrm{Pnma}$ & 44. \\
$\mathrm{Fe}_{2} \mathrm{As}$ & $\mathrm{Fe}_{2} \mathrm{As}$ & 3.627 & 3.627 & 5.973 & 90 & 90 & 90 & $\mathrm{P} 4 / \mathrm{nmm}$ & 45 \\
$\mathrm{Nb}_{4} \mathrm{As}_{3}$ & $\mathrm{Nb}_{4} \mathrm{As}_{3}$ & 3.516 & 14.660 & 18.830 & 90 & 90 & 90 & $\mathrm{Cmcm}$ & 46 \\
& $\alpha-\mathrm{V}_{4} \mathrm{As}_{3}$ & 3.4139 & 13.6798 & 18.0598 & 90 & 90 & 90 & $\mathrm{Cmcm}$ & 47 \\
$\mathrm{Cr}_{4} \mathrm{As}_{3}$ & $\mathrm{Cr}_{4} \mathrm{As}_{3}$ & 13.158 & 3.542 & 9.302 & 90 & 102.19 & 90 & $\mathrm{Cm}$ & 48 \\
$\mathrm{Mo}_{4} \mathrm{P}_{3}$ & $\mathrm{~B}_{4} \mathrm{~V}_{4} \mathrm{As}$ & 13.725 & 3.393 & 9.230 & 90 & 100.52 & 90 & $\mathrm{C} 2 / \mathrm{m}$ & 47 \\
$\mathrm{Rh}_{4} \mathrm{P}_{3}$ & $\mathrm{Mo}_{4} \mathrm{P}_{3}$ & 12.428 & 3.158 & 20.440 & 90 & 90 & 90 & $\mathrm{Pnma}$ & 49 \\
& $\mathrm{Rh}_{4} \mathrm{P}_{3}$ & 11.662 & 3.317 & 9.994 & 90 & 90 & 90 & $\mathrm{Pnma}$ & 50 \\
\hline
\end{tabular}


parameters, and a reference for the structural work. The only entries in this table are phases that have been structurally well-characterlzed, 1 .e.., their structure was determined by single-crystal technlques or by powder diffraction technlques if their structure type had previously been determined.

\section{Physical Properties}

Many of the physical properties of this layered class have not been measured using the precise techniques that might be desired. However, they do have a rather unique combination of general physical characteristics that make them interesting to the physical scientist. These phases are refractory with melting points in the range of 1150 to approximately $1600^{\circ} \mathrm{C}$. Conductivity measurements on arcmelted pellets of certain compounds indicated that these phases have electrical conductivity similar to that of the corresponding metals. Single crystals of these materials are characterized by bright, shiny faces indicative of metallic luster.

The single crystals of these materials are considerably more brittle than the corresponding metals. Under pressure crystals of these phases shatter into several smaller crystallites, while crystals of the corresponding metals exhlb1t malleab1lity under the same conditions. Pellets of these materials seem to be quite hard. A recent measurement 
of the hardness of $\mathrm{Ta}_{6} \mathrm{~S}$ was made by Mr. $\mathrm{H}$. Baker of the Ames Laboratory, and his measurement ylelded a Rockwell C hardness of 75 , indicating that $\mathrm{Ta}_{6} \mathrm{~S}$ has a hardness in the range of hardened steel.

\section{Common Structural Features}

1. Structural complexity

The structural complexity of the phases which are members of the layered class of compounds may best be illustrated by comparison with the structures of the $\mathrm{Me}_{1.0} \mathrm{X}$ phases, where Me represents a transition metal and $\mathrm{X}$. represents one of the group V or VI elements under consideration. The typical structure types observed for the one-to-one compounds are the $\mathrm{NaCl}$, NiAs or MnP structure types. Each of these is characterized by relatively small lattice parameters, high symmetry space groups, and high point symmetry for the metal atom positions in the structure. The metal coordination polyhedra correspond to octahedral or trigonal antiprismatic arrangements of near neighbors.

In contrast, the structures such as $\mathrm{TI}_{8} \mathrm{~S}_{3}, \mathrm{Nb}_{14} \mathrm{~S}_{5}$ or $\mathrm{Nb}_{5} \mathrm{P}_{3}$ have a much larger number of atoms in corresponding larger unit cells than the 1:1 phases. Space groups of the layered class of compounds are usually characterized by orthorhombic or lower symmetry. The polyhedra shown in Figures 2 and 4 indicate that the metal coordinations in 
$\mathrm{TI}_{2} \mathrm{~S}$ and $\mathrm{TI}_{8} \mathrm{~S}_{3}$ differ substantially from those found in the one-to-one type compounds in both the number and arrangement of metal and nonmetal nelghbors observed. Whereas the octahedral or trigonal antiprismatic arrangement of neighboring nonmetal atoms is predominant in the Me ${ }_{1.0} \mathrm{X}$ compounds, the layered class of compounds exhibits eight different metal coordination polyhedra. In the $\mathrm{NaCl}$ or NiAs structure type, the interatomic distances from a central metal atom to 1ts six nearest nelghbor nonmetal atoms are the same. Interatomic distances to near neighbors in the layered class of compounds are characterized by their varlation in bond length.

\section{Two layers of atiom positions}

The layered class of compounds is characterized by three closely related structural features. Table 8 illustrates that each phase is characterized by a crystallographic axis of approximately $3.4 \AA$. By contrast, the other crystallographic axes are normally much larger, where the greatest difference in axial length occurs for the $\mathrm{Ti}_{8} \mathrm{~S}_{3}$ structure.

For each of the structures in the layered class, all of the atoms occur in one of two planes which are perpendicular to the short axis. For most of the structures listed in Table 8, the arrangement of atoms in the two distinct layers is very closely related. Although a wide varlety of symmetry elements are inherent in the space groups for the structures 
Iisted, the most common is the presence of an inversion center midway between the layers of atoms (1gnoring the mirror plane discussed above). A second, somewhat less common symmetry element that relates the atom positions of one plane with the second plane is a c-centering operation. The presence of symmetry elements such as c-centering or inversion in the space group mean that the positioning of atoms in the two layers are not independent, but rather, the relative positions of atoms in each distinct layer is the same, except for a change in orientation of the two layers. Of the known examples, only the $\mathrm{Nb}_{8} \mathrm{P}_{5}, \mathrm{Mo}_{8} \mathrm{P}_{5}$ and $\mathrm{Fe}_{2} \mathrm{P}$ structure types do not exhibit this close relationship between the two layers. In each of the metal-rich layered structure types there is a mirror plane coincldent with the two layers of atoms. The close relationship between these three structural features, a short axis, two distinct layers of atoms, and a mirror plane coincident with the layers of atoms can be seen in the following way. An axis perpendicular to the planes of atom positions and substantially longer than $3.4 \AA$ would imply the presence of at least a third layer of atoms and the loss of a mirror plane in at least one of the atom layers. Conversely, if a mirror plane were not coincident with a particular atom layer, the atom positions would no longer be confined to planes, and, furthermore, atom positions above and below an approximate layer, should one exist, would be 
different, Implying a larger repeat distance for the unit cell in the direction perpendicular to the layers of atoms. In the discussion of the metal-rich layered structure types in terms of a qualitative bonding model that follows, an attempt will be made to use the presence of a mirror plane coincldent with the atom layers as a restraint upon the symmetries of the interactions between neighboring atoms.

\section{Nonmetal coordination polyhedron}

The predominant structural feature of the phases which are members of this layered class is the presence of capped trigonal-prismatic coordination for the nonmetal atom. The description of the metal coordination polyhedra that follows will 1llustrate the strong contrast between the metal atom, with the ablitty to form several different coordination polyhedra, and the nonmetal, which normally exists only the capped trigonal-prismatic coordination polyhedron. This contrast has suggested to a number of investigators that the key to understanding the chemical bonding in the layered type of compounds centers on understanding the role of the trigonal prism in the structures.

The significance of the trigonal prismatic coordination polyhedron has been discussed in several papers by Conard (1) and Franzen (51). Their discussion offers an explanation for the observed high coordination number of the nonmetal (six to nine metal atoms as near nelghbors) which is also 
consistent with the observed physical properties of the corresponding phases. Other Investigators, such as Aronsson, Lundström and Rundqvist (52), Hassler (39), and Lundström (37), emphasized the importance of the trigonal prism as a structural feature by describing the different structures in terms of differences in interconnections among the trigonal prisms. For the $\mathrm{Nb}_{2} \mathrm{Se}$ and $\mathrm{Nb}_{5} \mathrm{Se}_{4}$ structures the nonmetal coordination polyhedra, though not trigonal prismatic, are very closely related to trigonal prismatic coordination. For these two phases, the nonmetal coordination has been described by Conard, Norrby and Franzen (36) as incomplete trigonal prismatic where six of the seven atoms of a monocapped trigonal prism are present. The $\mathrm{Nb}_{4} \mathrm{As}_{3}$ structure type is the only member of the layered class of compounds for which one nonmetal atom position exhibits a coordination polyhedron other than trigonal prismatic or a fragment thereof.

The presence of the trigonal prismatic coordination polyhedra in these structure types is clearly important. Consideration of the trigonal prism places the emphasis on the contribution of the nonmetal atom to the formation of the Me-X bonds, The remainder of this thesis will be concerned with the same Me-X bonds, but the emphasis will be shifted to the role that the metal atom might play in their formation.

The structures under consideration here all contain more or less capped trigonal prismatic partial coordination 
polyhedra for the nonmetal atoms. The viewpoint adopted here is that it is the role of the metal bonding contribution that determines the physical packing of the nonmetal P.C.P. and thus, the differences in the different structure types under discussion.

4. Metal coordination polyhedra

Before considering the nature of the metal coordination polyhedra found in this class of compounds, it w1ll be important to consider in some detail what is meant by a coordination polyhedron. Typically, the coordination polyhedron of an atom is a pure geometrical concept and is defined as the three-dimensional arrangement of neighboring atoms about a central atom. Ideally, a coordination polyhedron would include those neighboring atoms which exhibit significant chemical interaction with the central atom of the polyhedron. For an isolated molecule, such as gaseous $\mathrm{CCl}_{4}$, the coordination polyhedron about the carbon atom is welldefined. The tetrahedrat arrangement of four chlorisie atoms represents the strong sigma bonds of the molecule, and the chemist feels comfortable in thinking that the interaction between carbon and chlorine electrons takes the form of tetrahedrally arranged $\mathrm{C}-\mathrm{Cl}$ chemical bonds.

In the solid, however, a portion of the electron density is known to be delocalized over the entire crystal. The Implication of this fact is that the chemical interaction 
between a central atom and its neighbors will not be limited to a small number of atoms. Intuit1vely, however, the degree of bonding or chemical interaction between two atoms is expected to depend, upon the distance between a central atom and its neighbors. The 1dentification of a certain group of atom positions as forming the coordination polyhedron for a particular central atom still requires that choices be made if one wishes to specify those atoms that form the strongest chemical bonds to the central atom. The choice of atoms forming a significant partial coordination polyhedron on the basis of an assumed interaction criterion will not imply that only the chosen atoms form chemical bonds to the central atom. Rather, it is the intent to select the partial coordination polyhedron on the basis that evidence, principally structural, provides an indication that the degree of chemical interaction can be expected to be greater for these polyhedral atoms.

Figure 5 represents a projection of the eight metal partial coordination polyhedra found in the layered class of compounds. Each partial polyhedron is labeled with a Roman numeral, I through VIII, which will be used throughout the subsequent discussion to identify the individual polyhedra. Table 9 represents the frequency of occurrence of each type of partial polyhedron in the structures of the metal-rich layered class and indicates the particular phases where each polyhedron ocoure. 

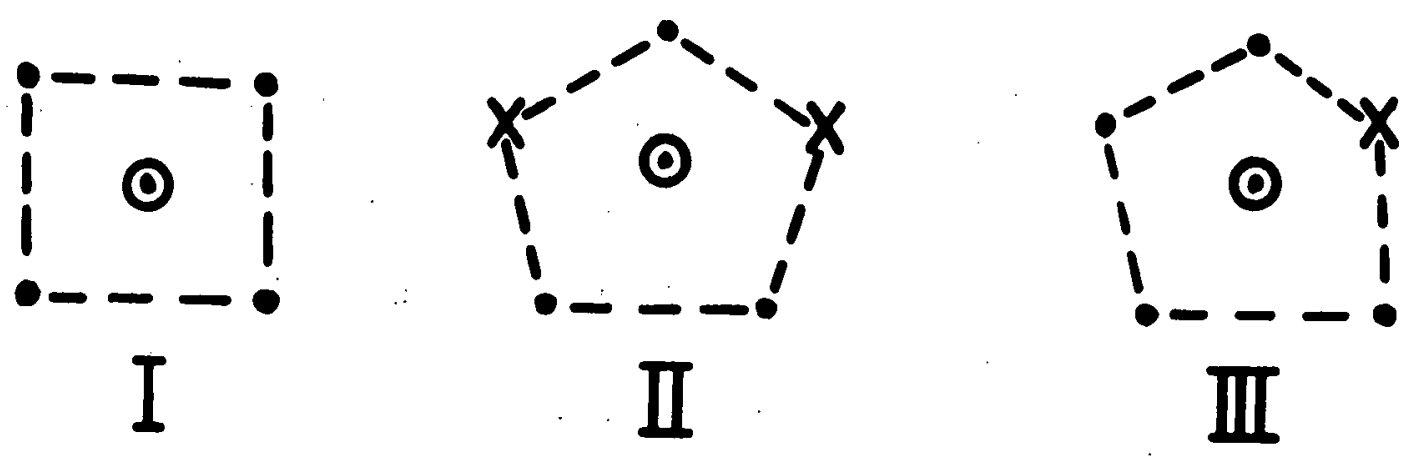

- 0
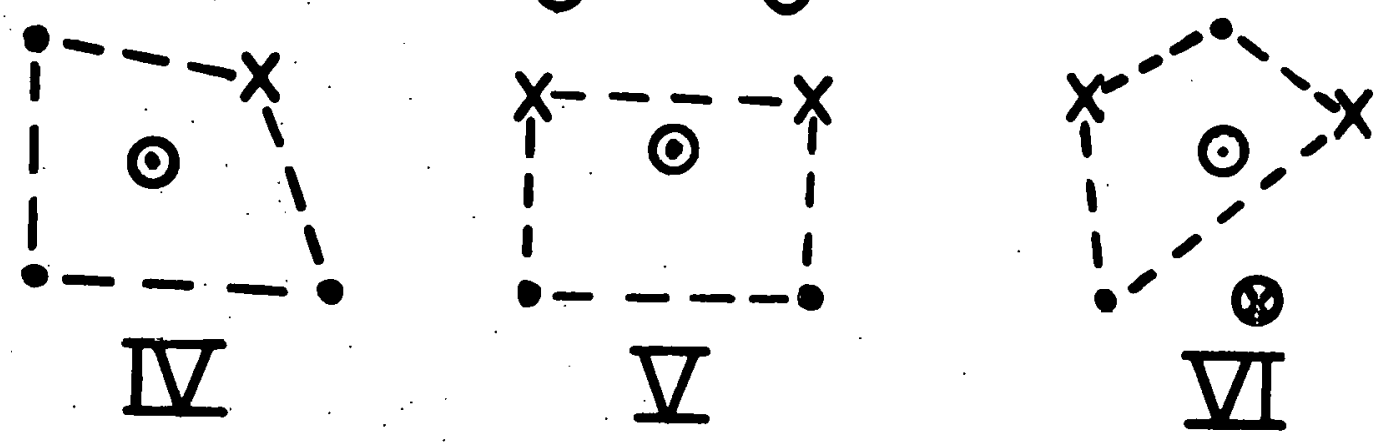

(

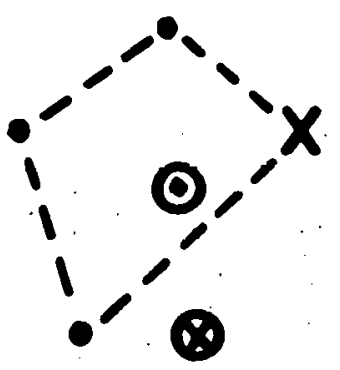

(
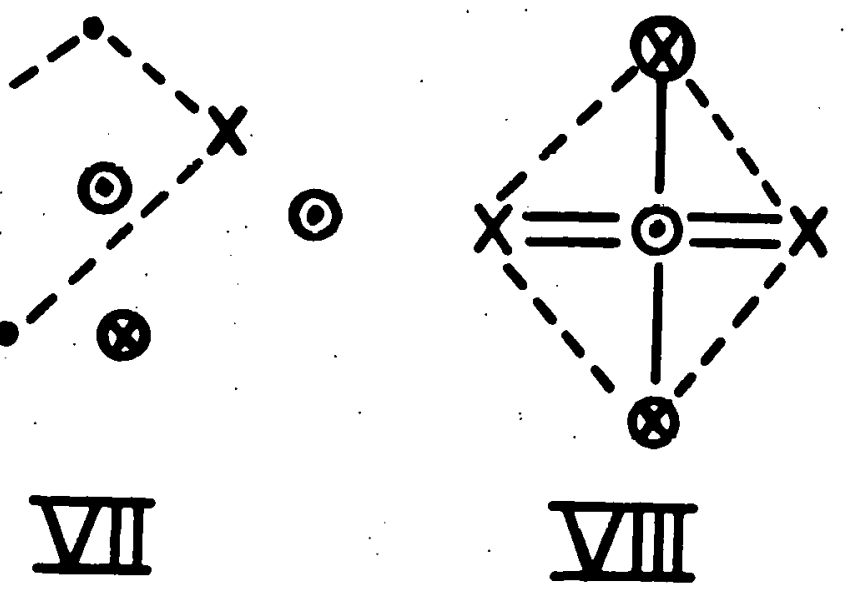

This numbering sequence will be used throughout the following discussion.

Figure 5. The metal Partial Coordination Polyhedra (P.C.P.) 
Table 9. Frequency of occurrence of metal Partial Coordination Polyhedra (P.C.P.) in the structures of the layered class

\begin{tabular}{|c|c|c|c|}
\hline $\begin{array}{l}\text { Metal } \\
\text { P.C.P. }\end{array}$ & $\begin{array}{l}\text { Number of Independent Atom } \\
\text { Pcsitions with this Type } \\
\text { of P.D.P. }\end{array}$ & $\begin{array}{l}\text { Structure Types where the } \\
\text { P.C.P. Occur }\end{array}$ & $\begin{array}{l}\text { Sto1chiometry } \\
\text { Range }\end{array}$ \\
\hline$I$ & $(16.5 \%)$ & $\begin{array}{l}\mathrm{Cr}_{4} \mathrm{As}_{3}, \mathrm{Nb}_{2} \mathrm{Se}, \mathrm{TI}_{2} \mathrm{~S}\left(\mathrm{Ta}_{2} \mathrm{P}\right), \\
\mathrm{TI}_{8} \mathrm{~S}_{3}, \mathrm{Nb}_{7} \mathrm{P}_{4}, \mathrm{Nb}_{8} \mathrm{P}_{5}, \\
\mathrm{Nb}_{5} \mathrm{P}_{3}, \mathrm{Nb}_{2} \mathrm{~S}_{8}, \mathrm{Nb}_{14} \mathrm{~S}_{5}, \\
\mathrm{Nb}_{4} \mathrm{As}_{3}, \mathrm{Nb}_{5} \mathrm{Se}_{4}\end{array}$ & $1.25 \leq \mathrm{Me} / \mathrm{X} \leq 2.8$ \\
\hline II & $\begin{array}{l}29 \\
(24.0 \%)\end{array}$ & $\begin{array}{l}\mathrm{Cr}_{4} \mathrm{As}_{3}, \mathrm{Ti}_{2} \mathrm{~S}\left(\mathrm{Ta}_{2} \mathrm{P}\right), \mathrm{TI}_{8} \mathrm{~S}_{3}, \\
\mathrm{Nb}_{7} \mathrm{P}_{4}, \mathrm{Nb}_{8} \mathrm{P}_{5}, \mathrm{Nb}_{5} \mathrm{P}_{3}, \\
\mathrm{Nb}_{21} \mathrm{~S}_{8}, \mathrm{Nb}_{14} \mathrm{~S}_{5}, \mathrm{Nb}_{4} \mathrm{As}_{3}\end{array}$ & $1.33 \leq \mathrm{Me} / \mathrm{X} \leq 2.80$ \\
\hline III & $(2.5 \%)$ & $\mathrm{Nb}_{17} \mathrm{~S}_{5}$ and $\mathrm{Mo}_{8} \mathrm{P}_{5}$ & $1.60 \leq \mathrm{Me} / \mathrm{X} \leq 2.80$ \\
\hline IV & $(17.1 \%)$ & $\begin{array}{l}\mathrm{Nb}_{2} \mathrm{Se}, \mathrm{V}_{12} \mathrm{P}_{7}, \mathrm{Fe}_{2} \mathrm{P}, \mathrm{CO}_{2} \mathrm{P}, \\
\mathrm{Fe}_{2} \mathrm{As}, \mathrm{TI}_{2} \mathrm{~S}\left(\mathrm{Ta}_{2} \mathrm{P}\right), \mathrm{Ti}_{8} \mathrm{~S}_{3}, \\
\mathrm{Nb}_{7} \mathrm{P}_{4}, \mathrm{Nb}_{5} \mathrm{P}_{3}, \mathrm{Nb}_{2} \mathrm{~S}_{8}, \\
\mathrm{Nb}_{14} \mathrm{~S}_{5}, \mathrm{Mo}_{8} \mathrm{P}_{5}\end{array}$ & $1.60 \leq \mathrm{Me} / \mathrm{X} \leq 2.80$ \\
\hline
\end{tabular}




\begin{tabular}{|c|c|c|c|}
\hline $\mathrm{v}$ & $(6.6 \%)$ & $\mathrm{V}_{12} \mathrm{P}_{7}, \mathrm{TI}_{8} \mathrm{~S}_{3}, \mathrm{Rh}_{4} \mathrm{P}_{3}$ & $1.33 \leq \mathrm{Me} / \mathrm{X} \leq 2.67$ \\
\hline VI & $\begin{array}{l}22 \\
(18.2 \%)\end{array}$ & $\begin{array}{l}\mathrm{Cr}_{4} \mathrm{As}_{3}, \mathrm{Nb}_{2} \mathrm{Se}, \mathrm{Hf}_{3} \mathrm{P}_{2}, \\
\mathrm{TI}_{2} \mathrm{~S}\left(\mathrm{Ta}_{2} \mathrm{P}\right), \mathrm{Nb}_{7} \mathrm{P}_{4}, \mathrm{Nb}_{5} \mathrm{P}_{3}, \\
\mathrm{Nb}_{8} \mathrm{P}_{5}, \mathrm{Nb}_{14} \mathrm{~S}_{5}, \mathrm{Mo}_{4} \mathrm{P}_{3}, \\
\mathrm{Nb}_{4} \mathrm{As}_{3}, \mathrm{Mo}_{8} \mathrm{P}_{5}, \mathrm{Nb}_{5} \mathrm{Se}_{4}\end{array}$ & $1.25 \leq \mathrm{Me} / \mathrm{X} \leq 2.80$ \\
\hline VII & $\begin{array}{l}13 \\
(10.7 \%)\end{array}$ & $\begin{array}{l}\mathrm{Nb}_{2} \mathrm{Se}, \mathrm{Hf}_{3} \mathrm{P}_{2}, \mathrm{Ti}_{2} \mathrm{~S}\left(\mathrm{Ta}_{2} \mathrm{P}\right) \\
\mathrm{Ti}_{8} \mathrm{~S}_{3}, \mathrm{Nb}_{5} \mathrm{P}_{3}, \mathrm{Nb}_{8} \mathrm{P}_{5}, \\
\mathrm{Nb}_{21} \mathrm{~S}_{8}, \mathrm{Nb}_{14} \mathrm{~S}_{5}, \mathrm{Mo}_{4} \mathrm{P}_{3}\end{array}$ & $1.60 \leq \mathrm{Me} / \mathrm{X} \leq 2.80$ \\
\hline VIII & $(5.8 \%)$ & $\mathrm{Cr}_{4} \mathrm{As}_{3}, \mathrm{Mo}_{4} \mathrm{P}_{3}, \mathrm{Nb}_{4} \mathrm{As}_{3}$ & $\mathrm{Me} / \mathrm{X}=1.33$ \\
\hline Other & $\begin{array}{c}\text { Partially filled } \\
\text { atom positions } \\
(1.7 \%)\end{array}$ & $\mathrm{Nb}_{8} \mathrm{P}_{5}\left(\mathrm{Nb}_{4}\right)$ and $\mathrm{Mo}_{4} \mathrm{P}_{3}\left(\mathrm{Mo}_{3}\right)$ & $1.33 \leq \mathrm{Me} / \mathrm{X} \leq 1.60$ \\
\hline $\begin{array}{l}\text { Total } \\
\text { Number: }\end{array}$ & 121 & & \\
\hline
\end{tabular}


Unit I represents the arrangement of eight metal atoms about a central atom and is often referred to as the cubic partial coordination polyhedron (P.C.P.) (partial because the atoms off the faces of the cube have not been Included in Figure 5 -- this exclusion of some capping atoms is continued throughout).

Unit IV is closely related to unit $I$ and can be described in terms of unit $I$ as the replacement of a Me-Me edge of the cube by an $\mathrm{X}-\mathrm{X}$ edge.

Unit II is the most commonly occurring metal P.C.P. having been observed for $24 \%$ of the metal atoms in the layered phases. Unit. II can be described in two different ways. The arrangement of ten metal and nonmetal nelghbors can be approximated by a pentagonal prism. An alternate method of description involves separation of the metal and nonmetal neighbors. Six metal atoms are arranged in the form of a distorted trigonal prismatic P.C.P. while the nonmetal atoms occur in a distorted square planar or square pyramidal P.C.P.

The arrangement of atoms in unit III is very similar to unit II where one of the $\mathrm{X}-\mathrm{X}$ edges of P.C.P. II has been replaced by a Me-Me edge in unit III. This particular polyhedron has only been observed for the $\mathrm{Mo}_{8} \mathrm{P}_{5}$ and $\mathrm{Nb}_{14} \mathrm{~S}_{5}$ structure types.

Unit $V$ is also very similar to unit II in the arrangement of neighboring atoms. In both units there is no 
difference in the number or orlentation of the nonmetal atoms of the polyhedron. In unit II the trigonal prismatic arrangement of six metal atoms occurs with the prism axis perpendicular to the plane of projection, while in unit $V$ the trigonal prismatic arrangement of metal atoms occurs with its prism axis parallel to the plane of the drawing.

The predominant feature of unit VIII is the arrangement of six nonmetal atoms in the form of a distorted octahedron. There are also (not shown) two to four additional metal atoms which occur at distances corresponding to the metal-metal interatomic distances of the polyhedra already described. Since the arrangement of these metal atoms off the faces and edges of the octahedron varies for the different phases where unit VIII occurs, their position (as with some capping atoms in units I-VII) is not specified in Figure 5. So far, this particular unit has only been observed for some of the $\mathrm{Me}_{4} \mathrm{X}_{3}$ phases.

The arrangement of nonmetal atoms in unit VI is very similar to the distorted octahedral arrangement of $\mathrm{X}$ atoms in VIII. One of the $X$ corners in unit VIII has been replaced by an Me-Me edge in unit VI. The orientation of the five remaining $X$ atoms in unit VI is the same as in unit VIII. The metal atom represented by a dot enclosed in a circle was Included as part of the P.C.P. here, because 1ts distance to the central atom is less than or equal to the interatomic 
distances from the central atom to the other metal neighbors depicted. In the figure.

Unit VII can be described in terms of either unit IV or unit VI. Its similarity to unit VI can be expressed as the replacement of one $X-X$ edge of unit VI by a Me-Me edge to form unit VII. The similarity to unit IV can be pictured as an expansion of one cube face formed by the $\mathrm{X}-\mathrm{X}$ edge and adjacent Me-Me edge to allow closer approach to the central atom of the metal and nonmetal atom in the same plane as the central atom. 


\section{STRUCTURAL ASPECTS OF THE METAL P.C.P.}

Thus far, the description of the metal P.C.P. and the earlier description of the $\mathrm{TI}_{2} \mathrm{~S}$ and $\mathrm{TI}_{8} \mathrm{~S}_{3}$ structures in terms of these P.C.P. have emphasized one of the key structural features of this layered class of compounds. The structure of any phase in this class is closely related to how the metal P.C.P. pack to fill space. Each of the structures under discussion here can be completely described in terms of face, corner, and edge sharing of the metal P.C.P. Alternately, each structure might be described in terms of networks of interconnected trigonal prismatic P.C.P. of the nonmetal atoms if one also allows space fllling by metal atoms between the various trigonal prisms. Th1s is the basic approach used by Aronsson, Lundström and Rundqvist (52) in discussing the structures of phosphides in this class. The approach of the Upsala school emphasizes the space fllling oboerved for most of these structure types.

The attempt to analyze the structures in the metal-rich layered class in terms of the metal P.C.P. rests upon the following assumptions: 1) that the capped trigonal-prismatic coordination polyhedra of the nonmetal atoms are in all cases compatible with the metal P.C.P. (observed to be the case for a wide variety of structures), and 2) that among the factors leading to the stability of an observed structure-type for a 
given stolchlometry and metal element, the differences in the coordination polyhedra of the nonmetal atoms (principally in the number of capping atoms) are unimportant relative to the differences in the metal P.C.P.

In viewing these structure types as the packing of metal partial coordination polyhedra, there are several aspects of the individual polyhedra that should be noted. Some of these aspects will be important, as they suggest certain limits for any qualitative description of the role that the metal may play in forming the Me-X bonds present in each structure. Other aspects are important for a better understanding of the structural similarities and differences between different structure types.

\section{A. A Relationship Between the}

Me and X P.C.P.

There are certain metal P.C.P., illustrated in Figure 6, that occur in known structure types of the metal-rich layered class only in conjunction with a nonmetal trigonal prism P.C.P. of particular orientation. Figure 6 shows the orientations of the nonmetal atom trigonal prismatic P.C.P. in the cases of linkage to units VI, VII and IV. For units VI and VII, the two trigonal prisms of different orientation share edges. The remaining nonmetal atom of unit VI is trigonal prismatic but occurs with its axis either 

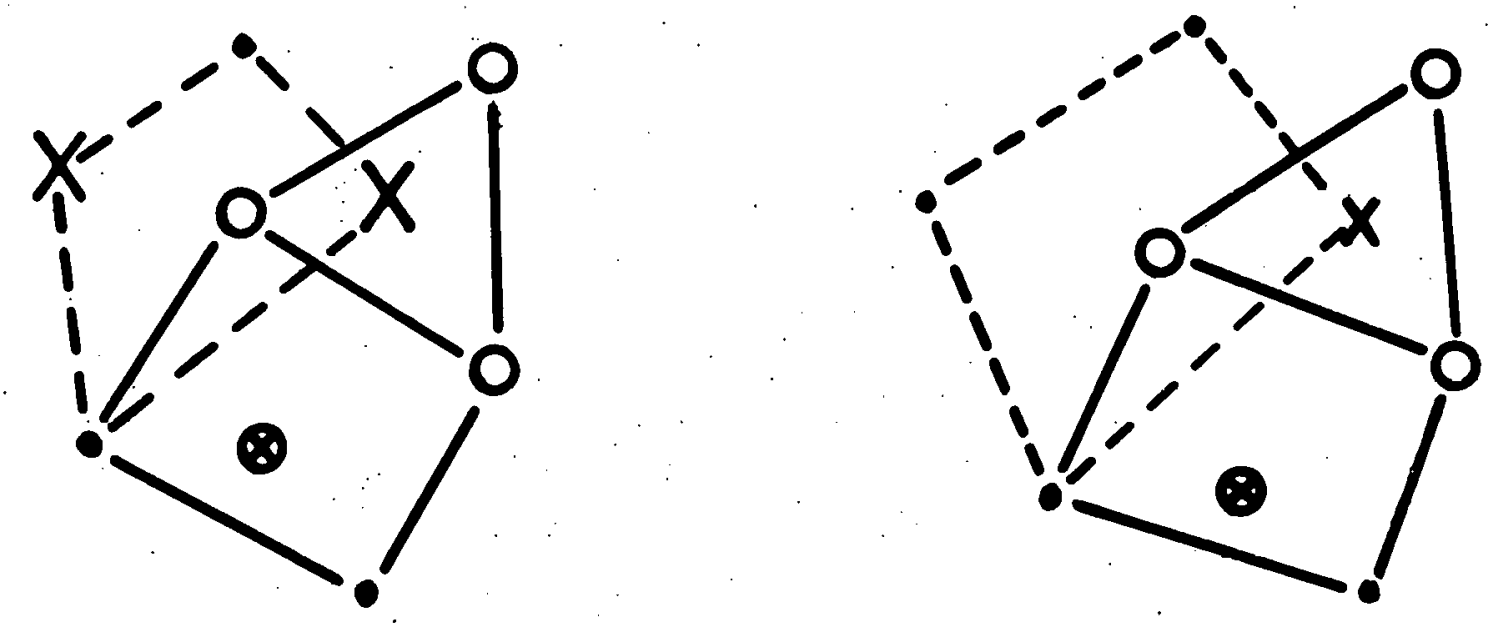

a) ZI

b) VIII

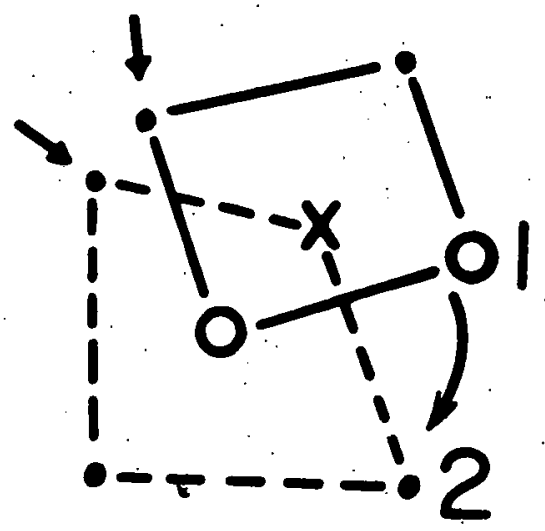

c)

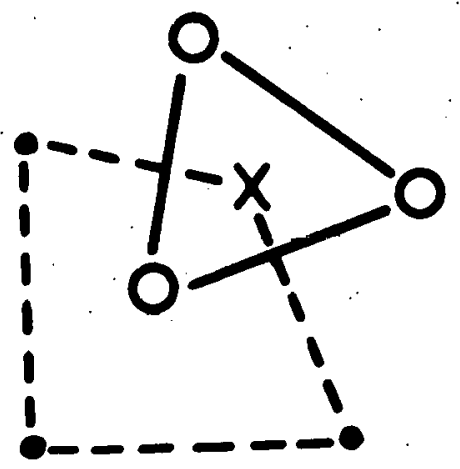

d) IV

Figure 6. An 1llustration of the relationship between certain metal partial coordination polyhedra (broken lines) and the nonmetal trigonal prisms (solid lines) 
perpendicular to the plane of the figure, as for $T 1(5)$ of $\mathrm{Ti}_{2} \mathrm{~S}$, or parallel to the plane of the figure, as for $\mathrm{Hf}(2)$ in the $\mathrm{Hf}_{3} \mathrm{P}_{2}$ structure.

The relationship between the occurrence of units IV, VI and VII and the prism orientation is important for several reasons. First, it emphasizes the partial structural determining nature of the metal coordination polyhedra and their linkages. That is, if the known structures are considered to provide all possible examples of interpenetrating P.C.P. Iinkages, the coordination polyhedra about an atom in one layer provides limits for the possible coordination polyhedra of atoms in the second layer. Second, the relationship between units VI and VII together with their interpenetrating trigonal prisms suggests a possible approach to solving an unknown structure which is believed to belong to the metal-rich layered class. This possibility is considered in greater detail in Chapter VI.

The coordination polyhedra VI and VII have in common linkages to nonmetal trigonal prismatic P.C.P. with axes parallel to the plane of atomic positions, while unit IV occurs only with a prism having its axis perpendicular to the atom plane. The lack of occurrence of unit IV linked with a nonmetal P.C.P. of parallel axis suggests one possible effect of packing considerations on the cholce of coordination polyhedra. Figure $6(\mathrm{c})$ indicates the hypothetical 
case of a parallel prism interpenetrating unit IV. Arrows indicate two metal atoms which would have an interatomic distance corresponding to expected repulsive interaction between the two atoms. Any attempt to change the orientation of the trigonal prism relative to unit IV by a rotation, represented by the curved arrow in the drawing, would decrease the repulsive overlap of the metal atoms indicated by the two arrows, but only at the expense of Increasing the repulsive overlap between atoms 1 and 2 .

\section{B. Packing of the Metal P.C.P.}

The drawings presented above in Figures 3 and 4 representing the $\mathrm{TI}_{2} \mathrm{~S}$ and $\mathrm{TI}_{8} \mathrm{~S}_{3}$ structures illustrate two ways that the metal P.C.P. pack to form a stable structure. An obvious question that arises from considering the various structure types in terms of the packing of metal P.C.P. concerns the number of possible structures that can be formed by linking the coordination polyhedra in different ways. In the comparison of the $\mathrm{Nb}_{14} \mathrm{~S}_{5}$ and $\mathrm{Nb}_{21} \mathrm{~S}_{8}$ structures which follows their simllarity will be emphasized by showing that they both have a common structural unit of appreclable size (24 P.C.P.), i.e., the structural unit consists of several Me and X P.C.P. sharing faces, edges, and corners in a particular manner. The significance of the recurrence of this particular structural unit in different structure types 
can only be appreciated in light of the large number of different units that would be possible based solely on packing considerations.

The number of possible structures implied by different linkings of the metal P.C.P. rapidly becomes very large, as can be seen by the following simple approach. A typical question to be answered 1s, "In the case of the most commonly occurring metal P.C.P., unit II, how many different ways can all elght of the polyhedra share faces with unit II?" Unit II has five faces, four of which contain both metal and nonmetal atoms and one face formed by four metal atoms. A second coordination polyhedron can share a face with unit II only if it has a face with a similar orientation of atoms as a face of unit II. For example, (Figure 5) units I and VIII cannot share a metal-nonmetal face with unit II since neither has a polyhedral face formed by two metal and two nonmetal atoms. Two P.C.P. of type II can share metal-nonmetal faces in only two different ways. Despite the fact that unit II has four faces of the same type, only two combinations of face sharing P.C.P. will yield distinct arrangements of atoms. Similar considerations were applied to all elght of the metal coordlnation polyhedra. There are ten possible ways that each of the four Me-X faces of unit II can share a face with the eight metal P.C.P., yielding a total of 40 different possibilities (ignoring 
Interferences between the Ilnked P.C.P.). Simllarly, there are ten ways the P.C.P. can share the single Me-Me face of unit II, for a total of 50 different orlentations of the elght P.C.P. for the five faces of unit II (again 1gnoring interferences).

The same considerations can be extended to each of the five P.C.P. which share one of their faces with unit II. Assume that each of the five units has an average of three remalning faces not shared with unit II, which can in turn share faces with other metal P.C.P. For unit II both types of faces (all metal or metal and nonmetal) implied ten possible ways of sharing faces with the eight P.C.P. Similar consideration for the 15 faces to be shared now would indicate roughly 150 possible ways of orienting the 15 metal P.C.P. Combining the 50 ways of orienting five metal P.C.P. about unit II with the 150 ways of orlenting the next 15 P.C.P. yields roughly 7,500 different possible structural units.

Similar considerations for a different cholce of the starting P.C.P., other than unit II, yleld a similar number of different units which can be formed by 20 P.C.P. In effect, there are approximately 5000 different ways that 20 P.C.P. could pack when only space filling restrictions are considered. The recurrence of large, identical structural units in two different structure types suggests 
that some criteria other than space filling considerations are needed to explain the recurrence of large structural units in different structures. It is the point of view adopted here that the recurrence of such units is not only related to the nearest neighbor chemical bonding interactions, but that differences in packing of these larger units and differences between similar units illustrate the similarities and differences in chemical interactions for different chemical systems.

It should be emphasized that by specifying the nature of the P.C.P. that share faces w1th one of the eight coordination polyhedra, the polyhedra of a large number of atoms centered in the second layer of atoms have also been specified. In the $\mathrm{Ti}_{2} \mathrm{~S}$ and $\mathrm{Ti}_{8} \mathrm{~S}_{3}$ structures there are a total of six metal positions that have polyhedra of type $I$. Reference to Figure 7 indicates that the arrangement of polyhedra that share faces, edges, and corners with unit I is different for each of the six atom positions with P.C.P. of type I. Once these polyhedra are specified (solid lines), the polyhedra of those metal atoms which form the cubic arrangement of atoms in unit $I$ are also specifled (broken lines). It is the interdependence of the packing of the P.C.P. In the two layers of atoms that emphasizes the three dimensional nature of the compounds in this structural class. 


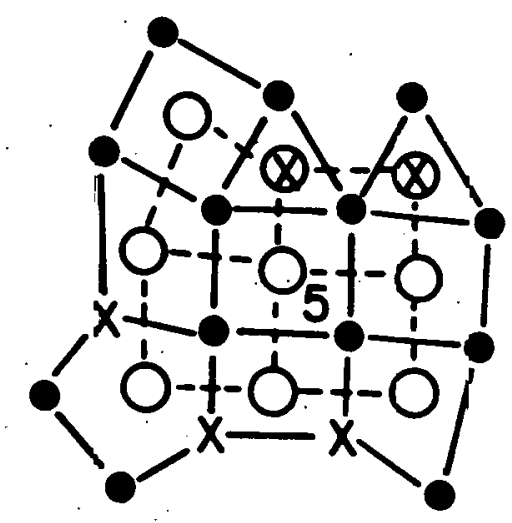

a) $\mathrm{Ti}$ (5)

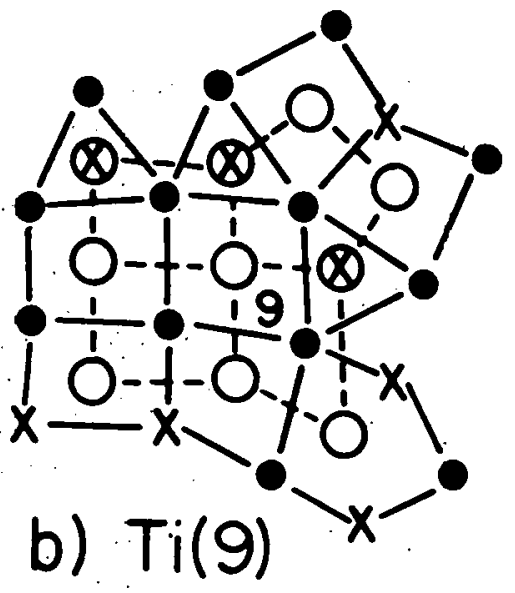

e) $\mathrm{Ti}(14)$

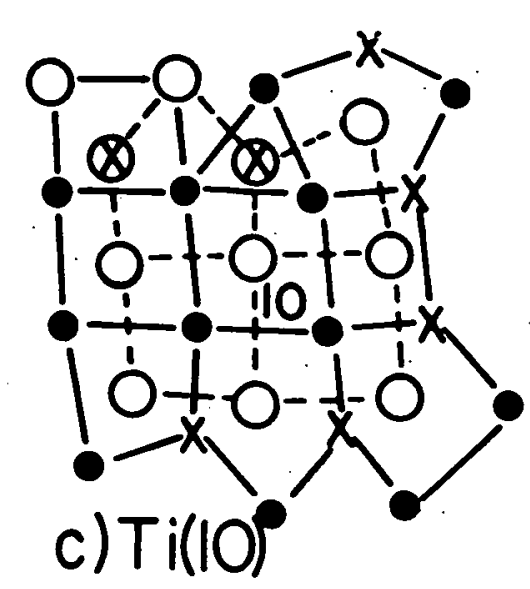

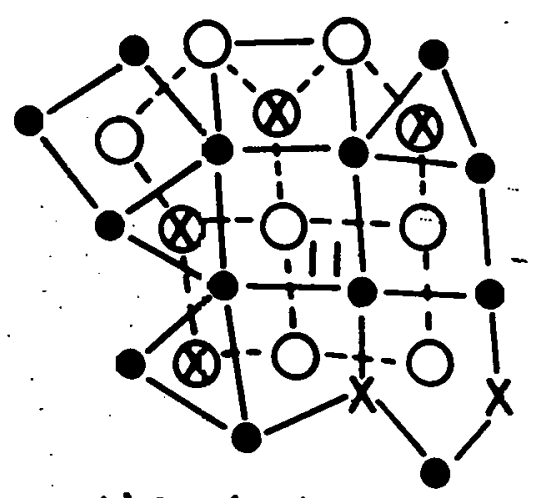

d) $\mathrm{Ti}(\mathrm{II})$
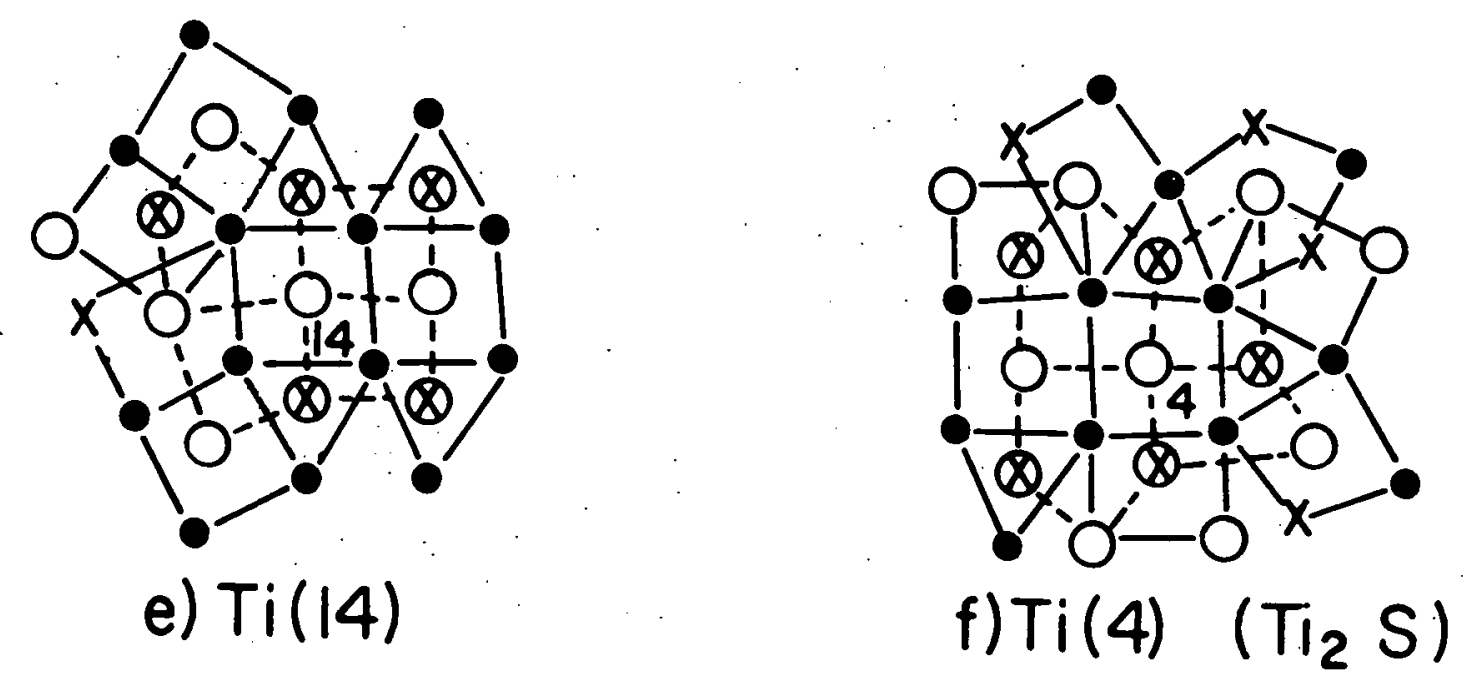

Figure 7. Interrelationship between face, edge, and corner sharing of metal P.C.P. In one layer (solld lines) with the type of metal P.C.P. In the adfacent layer (broken lines) 
In viewing the structures of this class in terms of the packing of metal and nonmetal P.C.P. It is important to keep in mind the interdependence of the P.C.P. of any atom position with the type of P.C.P. of its neighboring atom positions. In a larger sense, it is this interdependence that emphasizes the long range interaction between atoms in the structures of the layered class. If the occurrence of a particular P.C.P. Is in part dependent upon the nature of the P.C.P. of 1ts near nelghbors, then the P.C.P. of these near neighbors is in turn dependent upon the P.C.P. of their near neighbors, some of which will be second near neighbors for the original atom. This type of approach is obviously not limited to second near neighbor interaction but qualitatively extends throughout the entire structure. The long range Interaction between atoms can thus be thought of in terms of the interdependence between the various P.C.P. forming a particular structure type.

C. Me-X Bonds in P.C.P. I trrough VIII

If the metal partial coordination polyhedra illustrated in Figure 5 above are separated into the Ir Me-Me and Me-X components, the P.C.P. can be classified in terms of the number and orientation of nonmetal nelghbors. Figure 8 illustrates the position of the nonmetal atoms for all eight of the metal P.C.P. except unit I. In each of the figures the solid lines represent the number of Me-X inleratomic 

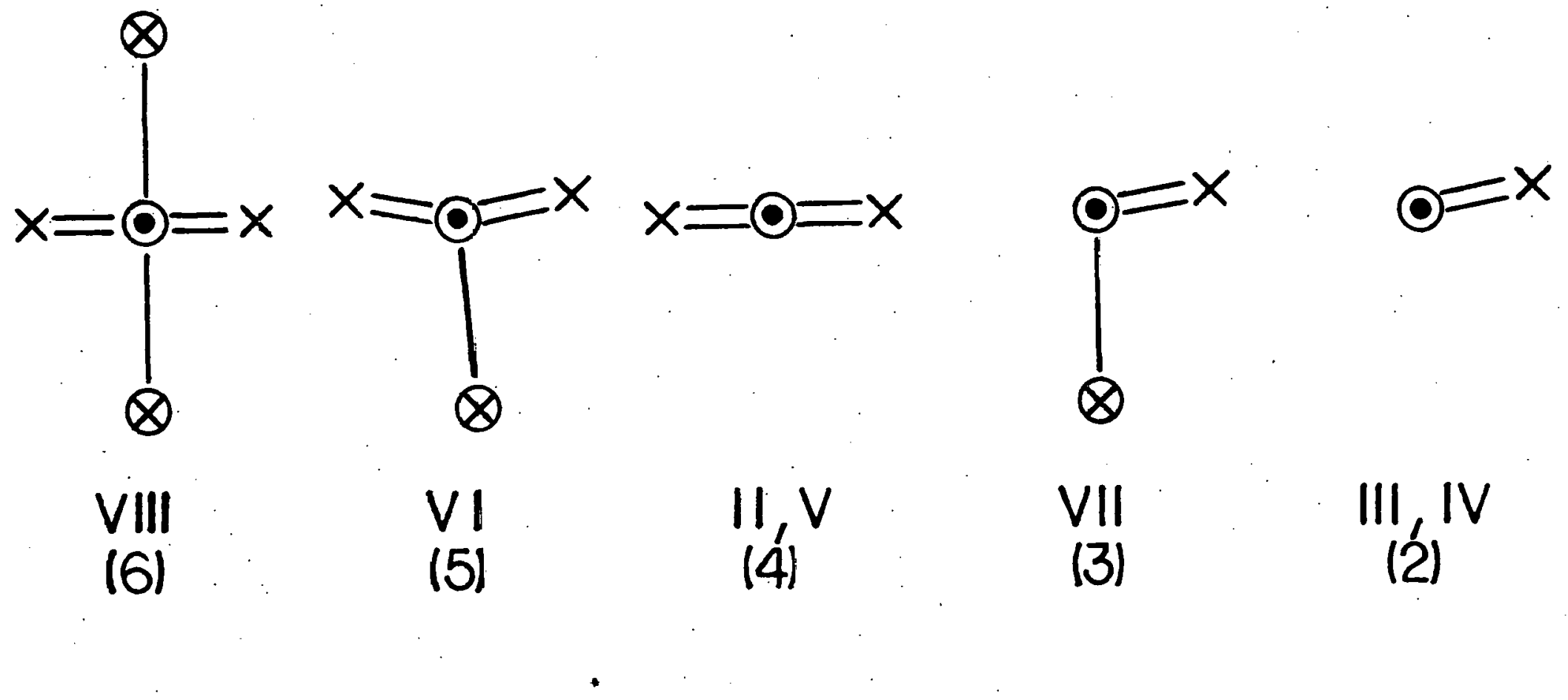

Figure 8. Number and orientation of Me-X bonds for metal P.C.P. II through VIII. Numbers of Me- $\bar{X}$ bonds in parentheses 
vectors where two ines from the central atom to a nonmetal (X) represents two separate vectors to nonmetal atoms above and below the plane of the central metal atom. For four Me-X vectors, units II and $V$ have the same orientation of the four vectors despite a difference in orientation of metal neighbors. Units III and IV differ in both the number and orientation of metal atoms in the P.C.P., as can be seen in Figure 5 above.

Figure 8 illustrates that the number and orientation of the Me-X vectors for P.C.P. II through VII can be described In terms of the arrangement of Me-X vectors of the octahedral coordination polyhedron, unit VIII. This drawing illustrates two interesting points. If a metal P.C.P. contains.less than six metal-nonmetal vectors, then the corresponding orientation of Me-X bonds for the central atom can be described as part of the octahedral orlentation of vectors seen in unit VIII. The second point concerns the fact that for 19 different structure types which have 99 Independent metal atoms whose polyhedra contain nonmetal atoms, never does the arrangement of $X$ atoms differ from those shown in Figure 8 (unless capping atoms are included). There are certainly other possible arrangements of vectors from metal to nonmetal atoms with between five and two nonmetal neighbors that would be compatible with the features of this structural class, but they have not yet been observed. 
The similarity between the orlentation of the Me-X vectors for P.C.P. II through VII and the Me-X vectors of unit VIII raises questions concerning a possible relationship between the bonding in unit VIII and the bonding in the other P.C.P. In the discussion that follows, a possible bonding scheme will be presented to account for the octahedral arrangement of $\mathrm{X}$ atoms in P.C.P. VIII, and this scheme will later be applied to the Me-X bonds of the other partial coordination polyhedra.

D. The Same P.C.P. for Different Metals

Table 9 lists the varlous structure types for which each of the individual coordination polyhedra occurs. Units I, II, IV, VI, and VII all occur for several different structure types with a wide range of stolchiometry and for a wide varlety of metal-nonmetal systems. For example, unit II occurs for the $\mathrm{Cr}_{4} \mathrm{As}_{3}, \mathrm{Ta}_{2} \mathrm{P}, \mathrm{TI}_{8} \mathrm{~S}_{3}, \mathrm{Nb}_{7} \mathrm{P}_{4}, \mathrm{Nb}_{8} \mathrm{P}_{5}, \mathrm{Nb}_{5} \mathrm{P}_{3}$, $\mathrm{Nb}_{21} \mathrm{~S}_{8}, \mathrm{Nb}_{14} \mathrm{~S}_{5}, \mathrm{Nb}_{4} \mathrm{As}_{3}, \mathrm{Hf}_{3} \mathrm{P}_{2}, \mathrm{~V}_{12} \mathrm{P}_{7}, \mathrm{Fe}_{2} \mathrm{P}, \mathrm{Co}_{2} \mathrm{P}, \mathrm{Fe}_{2} \mathrm{As}$, $\mathrm{Mo}_{4} \mathrm{P}_{3}$ and $\mathrm{Mo}_{8} \mathrm{P}_{5}$ structure types. The viewpoint adopted here is that a qualitative bonding model that is developed with the purpose of enhanced understanding of the structures of this class should account in some way for the recurrence of the specific coordination polyhedra, despite differences in the nature of the various metal atoms, 1 .e., differences in size, number of electrons, electronic configuration, etc. 
From a slightly different point of view, each particular type of polyhedron can be thought of as requiring a certain arrangement of effective bonding directions before the unit can occur. The chemist normaliy would assoclate a speciflc arrangement of atoms or bonds with some particular bond character of the central atom. Since several different metals form the same arrangement of bonds to neighbor atoms, the question to be considered is what is the bond character or tendency of forming chemical bonds that is common to the different metals.

\section{E. Different P.C.P. for the Same Metal}

Most of the transition metals that combine to form binary phases which belong to the layered structural class are capabie of forming a wide variety of metal P.C.P. for a given structure type. For example, the P.C.P. of titanium in the $\mathrm{Ti}_{8} \mathrm{~S}_{3}$ structure can be represented by units $I(5)$, $\operatorname{II}(3), \operatorname{IV}(3), V(3)$, and $\operatorname{VII}(2)$, where the numbers in parentheses represent the number of different independent titanium positions that have the corresponding type of P.C.P. Using the same notation, niobium positions in $\mathrm{Nb}_{14} \mathrm{~S}_{5}$ can be represented by $\mathrm{I}(4), \operatorname{II}(2), \operatorname{III}(1), \operatorname{IV}(3)$, $\left.V I(]_{1}\right)$, and $\operatorname{VII}(3)$. The listing of structure types and the polyhedra in Tables 8 and 9 further substantiates the fact that many of the metals are capable of forming several different metal conrination polyhcdra. 
A metal atom that forms P.C.P. as different as units I and VIII shows considerable flexibility in the formation of Its chemical bonds. One question ralsed here concerns the nature of the metal atom that allows this flexibility in the formation of its bonds.

$$
\begin{gathered}
\text { F. Occurrence of the Me P.C.P. } \\
\text { and the Me/X Ratio }
\end{gathered}
$$

As emphasized above, the comparison of the structures of the layered class under consideration involves the comparison of the various bullding blocks or polyhedra that combine to form the structures. Since the various P.C.P. differ in the number of their metal and nonmetal atoms, it is natural to ask if there is a correlation between the stoichiometry of a particular phase and the ratio of the number of metal and nonmetal atoms of the various coordination polyhedra observed for the phase. Table 9 indicates the stolchiometry range over which the various metal coordination pulyhedra occur. There is certainly a not unexpected general trend that those polyhedra containing a relatively large number of metal atoms often occur in the more metal rich phases, such as $\mathrm{Nb}_{21} \mathrm{~S}_{8}, \mathrm{Nb}_{14} \mathrm{~S}_{5}$, and $\mathrm{TI}_{8} \mathrm{~S}_{3}$, while P.C.P., such as VIII and VI, are found in phases with relatively low $\mathrm{Me} / \mathrm{X}$ ratios, such as $\mathrm{Mo}_{8} \mathrm{P}_{5}, \mathrm{Nb}_{8} \mathrm{P}_{5}$ and the $\mathrm{Me}_{4} \mathrm{X}_{3}$ phases. What is interesting is the occurrence of 
unit I in the structures of the compounds $\mathrm{Me}_{4} \mathrm{X}_{3}, \mathrm{Nb}_{5} \mathrm{Se}_{4}$, etc. with low Me/X ratios, and the occurrence of unit VI (with five nonmetal neighbors) in the $\mathrm{Nb}_{14} \mathrm{~S}_{5}$ structure. These cases Indicate that the occurrence of the different Me P.C.P. in a compound depends on other factors rather than solely on the Me/X ratio. 


\section{PARTIAL COORDINATION POLYHEDRA \\ AND INTERATOMIC DISTANCES}

\section{A. Introduction}

In the discussion of the polyhedra that occur for phases In the layered class of compounds reference was made to a very important characteristic of the polyhedra which requires expansion here. This characteristic is the variation in the interatomic distances between a central atom and the atoms forming the coordination polyhedron. This variation in interatomic distances will be illustrated for units II, IV and the trigonal prisms which occur for phases in the $\mathrm{Nb}-\mathrm{S}$, $\mathrm{Nb}-\mathrm{P}$ and T1-S systems. These systems w1ll illustrate trends that are true for all. of the phases in the layered class. The large number of structure types precludes listing the interatomic distances for all of the Individual phases forming this layered structural class.

There are certain trends based on differences in the interatomic distances that hold desplte changes in structure type, $\mathrm{Me} / \mathrm{X}$ ratio and nature of the Me-X system. These trends suggest possible differences in the chemical bonds involved, and an attempt will be made in section VII.E., below, to correlate suggested chemical differences. with a qualitative bonding model.

It should be noted that differences in bond angles for the varlous P.C.P. were never directly compared. It was noted 
In superimposing drawings of different structure types that the geometries of given polyhedra did vary, but the reasons for such variations will not be pursued further.

\section{B. Variation in Interatomic Distances}

1. Metal partial coordination polyhedra

Tables 10 and 11 list the interatomic distances for the two metal P.C.P. which are illustrated in Figure 9. The numbering of atoms in this figure corresponds to the column headings of Tables 10 and 11 . For unit IV the only comparison that will be made is between the three metal-metal interatomic distances listed in the last three columns of Table 10. For unit II the Me-X interatomic distances listed in the columns corresponding to atom 1 and 2 of Table 11 should be compared and the Me-Me distances in the columns headed by atom 3, 4, and 5 should be compared.

The comparison of interatomic distances such as those listed in these two tables is subject to certain limitations. In the discussion that follows, the Me-Me bond lengths and Me-X bond lengths will not be compared, and bond lengths will not be compared for the different metal-nonmetal systems.

The second entry for each of the columns in Tables 10 and 11 is the Pauling bond order corresponding to the interatomic distance listed in the same column. These bond orders 
Table 1.0. Variability of Interatomic distances for P.C..P. IV

\begin{tabular}{|c|c|c|c|c|c|c|c|}
\hline \multirow{2}{*}{ Phase } & \multirow{2}{*}{$\begin{array}{l}\text { Number } \\
\text { Central }\end{array}$} & \multirow{2}{*}{$\begin{array}{l}\text { of } \\
\text { Atom. }\end{array}$} & & \multicolumn{4}{|c|}{ Atom Number } \\
\hline & & & & 1 & 2 & 3 & 4 \\
\hline $\mathrm{T} 1_{2} \mathrm{~S}$ & $\mathrm{~T} 1(3)$ & & & $2.438 / .75$ & $2.853 / .46$ & $2.793 / .57$ & $2.890 / .40$ \\
\hline $\mathrm{T}_{8} \mathrm{~S}_{3}$ & $\mathrm{~T} 1(2)$ & & & $2.497 / .60$ & $2.803 / .55$ & $2.793 / .57$ & $2.783 / .60$ \\
\hline $\mathrm{T}_{8} \mathrm{~S}_{3}$ & $\mathrm{~T} 1(3)$ & & & $2.454 / .71$ & $2.868 / .43$ & $2.881 / .41$ & $2.960 / .30$ \\
\hline $\mathrm{T} \div 8 \mathrm{~S}_{3}$ & $\mathrm{TI}(8)$ & & & $2.460 / .69$ & $2.910 / .37$ & $2.875 / .42$ & $2.767 / .63$ \\
\hline & mean & $\mathrm{T} 1-\mathrm{S}$ & $=$ & 2.474 & & mean $\mathrm{T} 1-\mathrm{T} 1=$ & 2.848 \\
\hline & & $\sigma$ & $=$ & 0.020. & & $\sigma=$ & 0.060 \\
\hline $\mathrm{Nb}_{5} \mathrm{P}_{3}$ & $\mathrm{Nb}(8)$ & & & $2.502 / .79$ & $2.967 / .33$ & $3.015 / .22$ & $2.848 / .53$ \\
\hline $\mathrm{Nb}_{7} \mathrm{P}_{4}$ & $\mathrm{Nb}(4)$ & & & $2.461 / .93$ & $2.980 / .32$ & $2.916 / .41$ & $2.894 / .44$ \\
\hline & mean & $\mathrm{Nb}-\mathrm{P}$ & - & 2.482 & & mean $\mathrm{Nb}-\mathrm{Nb}=$ & 2.947 \\
\hline & & $\sigma$ & $=$ & 0.029 & & $\sigma=$ & 0.079 \\
\hline
\end{tabular}


Table 11. Variab1lity of interatomic distances ( $\AA$ ) for P.C.P. II

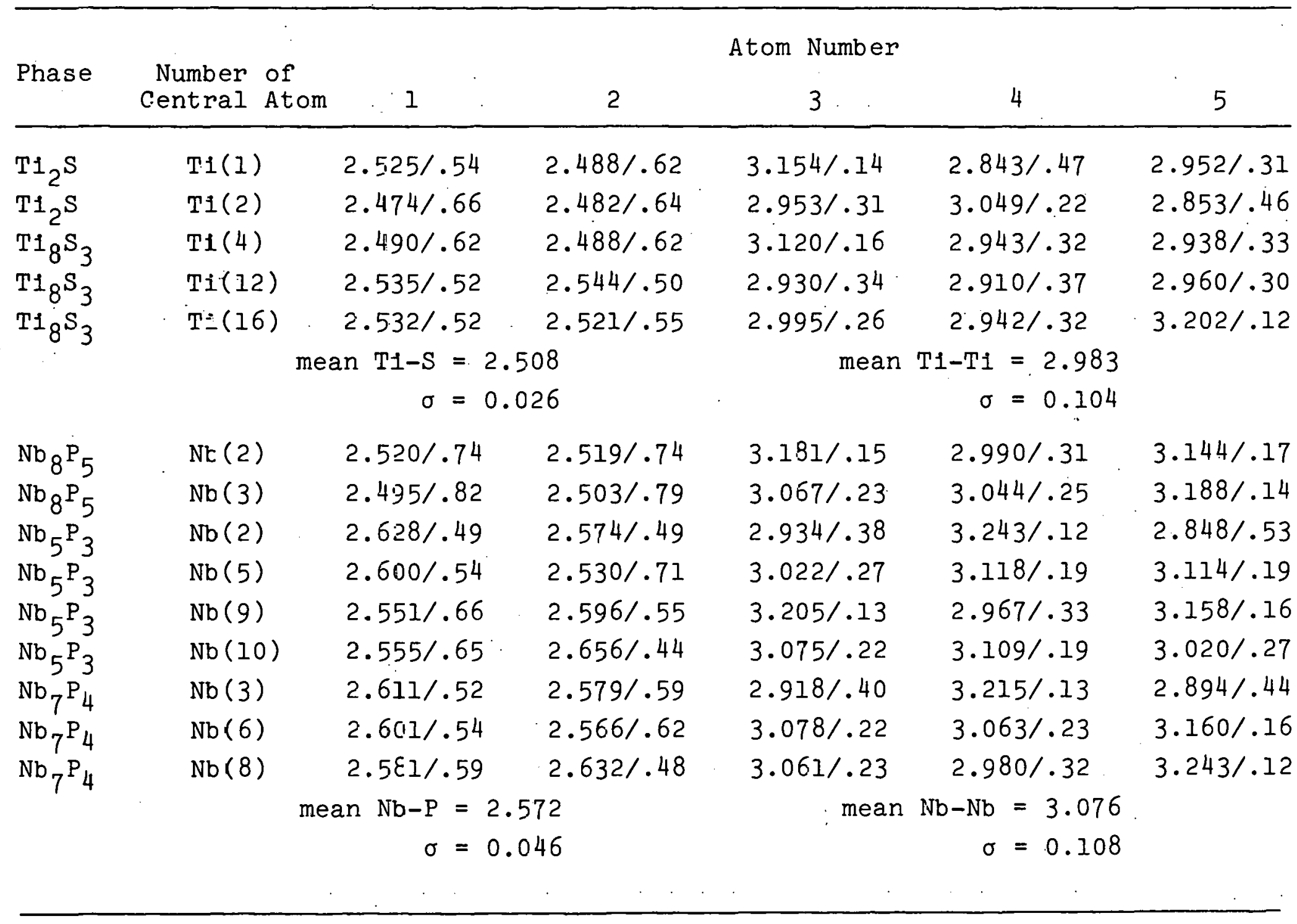



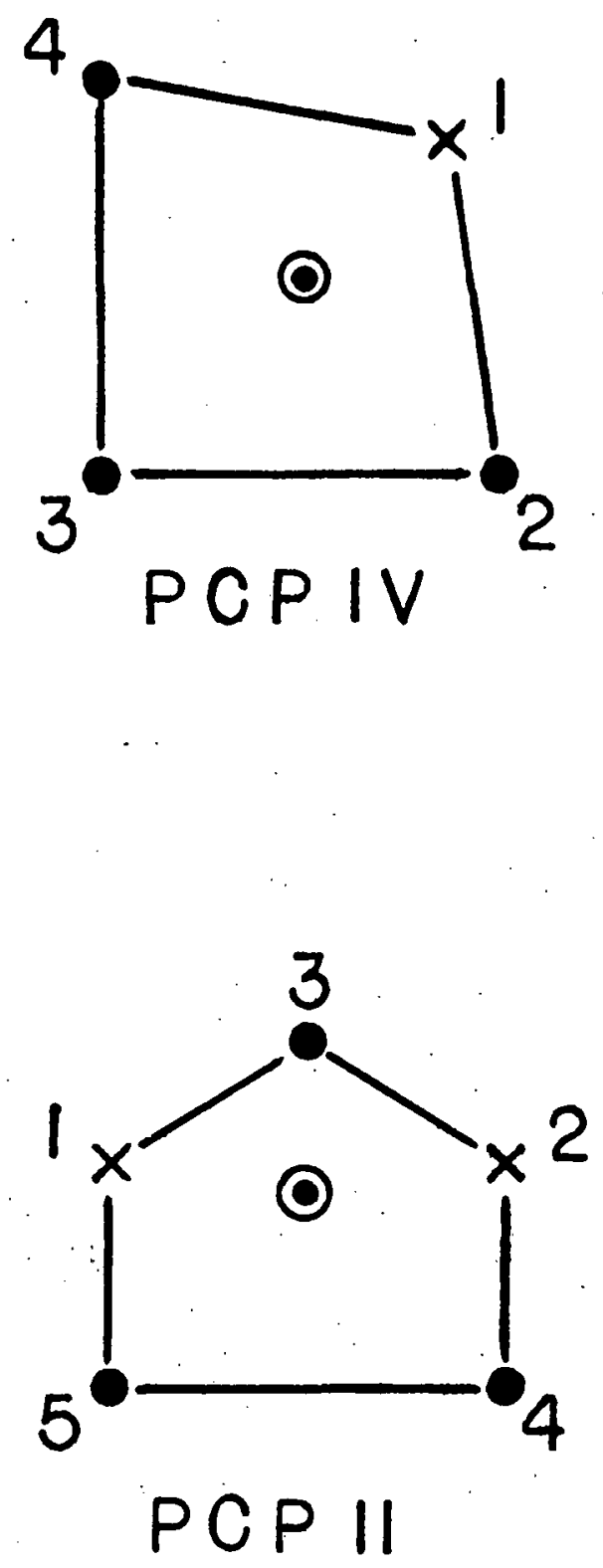

Figure 9: Atom numbering for P.C.P. II and IV in Table 10 and Table 1l, respectively 
were calculated using Pauling's empirical bond order-bond distance formula (53)

$$
D(n)=D(1)-0.600 \log n
$$

In this equation, $n$ corresponds to the bond order, $D(n)$ is the interatomic distance, and $D(1)=R(I)_{M e}+R(I)_{M e}$ or $X \cdot$ The values used for $R(1)$ were Pauling's single bond radii. The Pauling bond orders are included in the tables to accentuate the approximately exponential dependence of bond strength on the difference in bond length. The actual numbers for the various bond orders will not be considered to have significance in the following discussion.

In considering the interatomic distances of Tables 10 and Il, a question can be raised concerning how large a difference in interatomic distances must be before it corresponds to a chemically significant difference in bond strength. Chemical intuition suggests that, given a particular configuration of atoms, the bonding interaction between two atoms should he dependent upon the interatomic distance between those atoms. Similarly, the larger the difference in two interatomic distances, the greater the likelihood that the two distances will correspond to a difference in the strength of the chemical bond.

In Table 10 the three independent metal atom positions in unit IV for the Ti-S system have a minimum T1-Ti interatomic distance of $2.767 \AA$ and a maximum of $2.960 \AA$. The 
difference between these values of $0.193 \AA$ suggests that there may be significant differences in the strength of these two chemical bonds. A general survey of Table 10 indicates that the various Me-Me bond lengths for unit IV In $\mathrm{TI}_{2} \mathrm{~S}$ and $\mathrm{TI}_{8} \mathrm{~S}_{3}$ exhibit considerable variability between the two extreme values. The difference in standard deviation, $0.060 \AA$ for Ti-T1 and $0.020 \AA$ for Ti-S interatomic distances, accentuates the larger variation in Ti-Ti bond lengths of P.C.P. IV. Similar variation in the Me-Me bond distances for P.C.P. II can be seen in Table 1I. For both the T1-S and $\mathrm{Nb}-\mathrm{P}$ system the standard deviation of the Me-Me bond distances is substantially greater than the standard deviation of the Me-X bonds. Comparison of $\sigma$ values for Me-Me distances in unit II and IV indicates that the variability in Me-Me bonds in substantially greater in unit II than in unit IV. The trends illustrated by P.C.P. II and IV in the Ti-S and $\mathrm{Nb}-\mathrm{P}$ systems, $\underline{1} \cdot \underline{\mathrm{e}} .$, the greater varlability of Me-Me bond distances relative to the Me- $X$ distances and differences In Me-Me bond variability for different types of P.C.P., are characteristics that are common to each type of P.C.P. and common for the different metal-nonmetal systems which form otructures in the layered class. It is the greater variability in Me-Me bond lengths relative to the Me-X bond lengths in a metal P.C.P. which suggests the separation of metal bonding contribution between Me-X and Me-Me bonds 
discussed above. This separation is based upon the contention that the relatively fixed Me-X bonding contribution of the metal is more important than the relatively variable Me-Me bonding contribution.

\section{Trigonal prismatic partial coordination polyhedra}

Figure 10 indicates the numbering system for the independent atom positions for both the parallel and perpendicular orientations of the trigonal prism. This numbering system is used to identify each of the X-Me bonds in Table 12 . For the trigonal prism with axis perpendicular to the plane of. projection in Figure $10(a)$, atoms 1,2 , and 3 represent the six atoms forming the prism, while atoms 4 and 5 represent the two capping atoms off the faces of the prism which have the shortest interatomic distance to the central nonmetal atom. Atoms 1, 2, 3, and 4 correspond to the six atoms that form the trigonal prism with axis parallel to the projections plane of the figure, while atom 5 corresponds to the single capping atom found for prisms with this orientation.

The data in the table indicate that the variation in bond distances found for unit II and IV is also evident for the trigonal prism. Here the differences in Me-X bond lengths are larger than observed for the Me-X bond lengths of unit II above. The mean interatomic distances in the table indicate that the distance to capping atoms of the perpendicular prism often differ substantially from the distance to the six atoms 

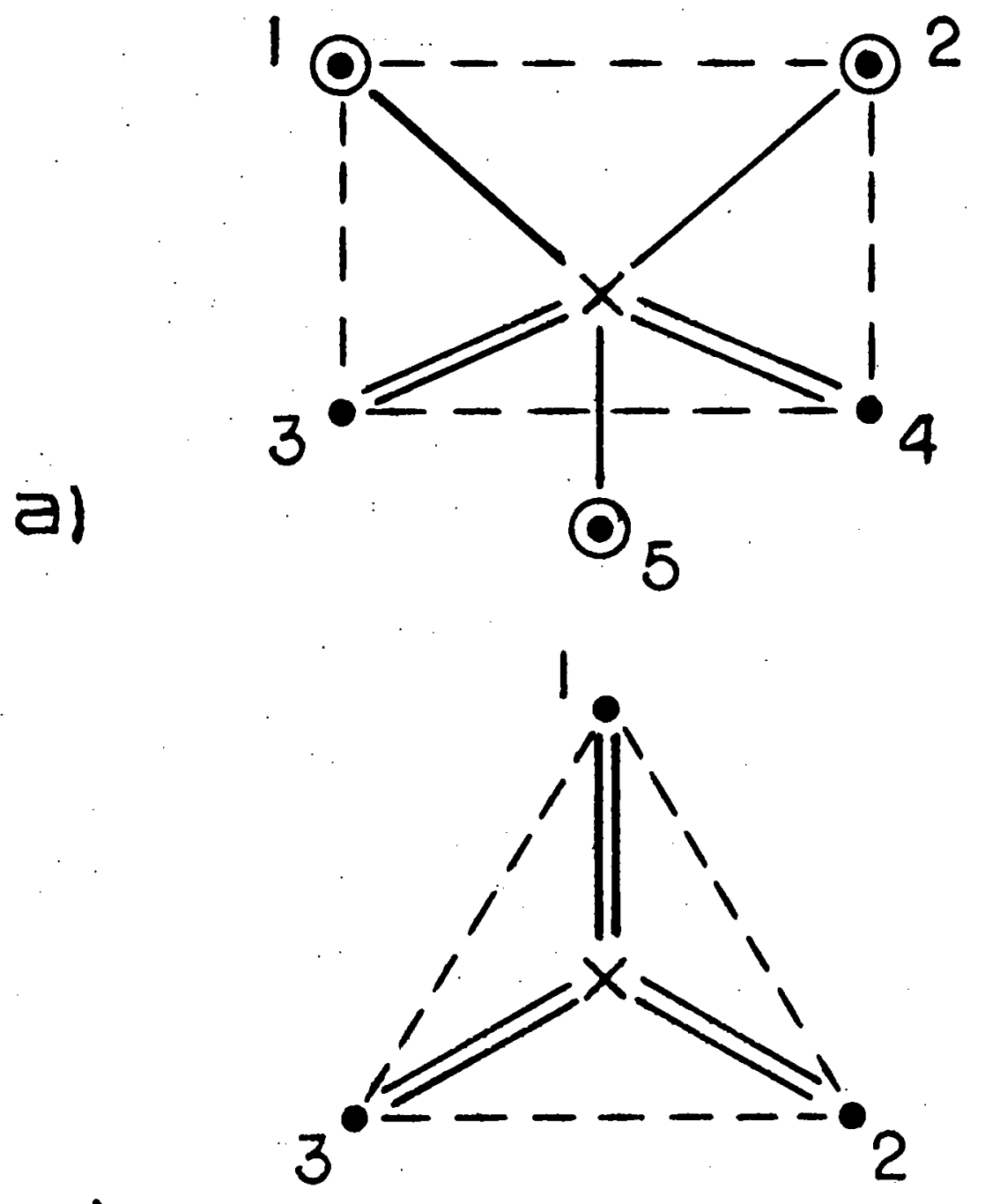

b)

Figurc 10. Atom numbering sequence for parallel and perpendicular prisms in Table 12 
Table 12. Me-X Interatcmic distances for the trigonal prism

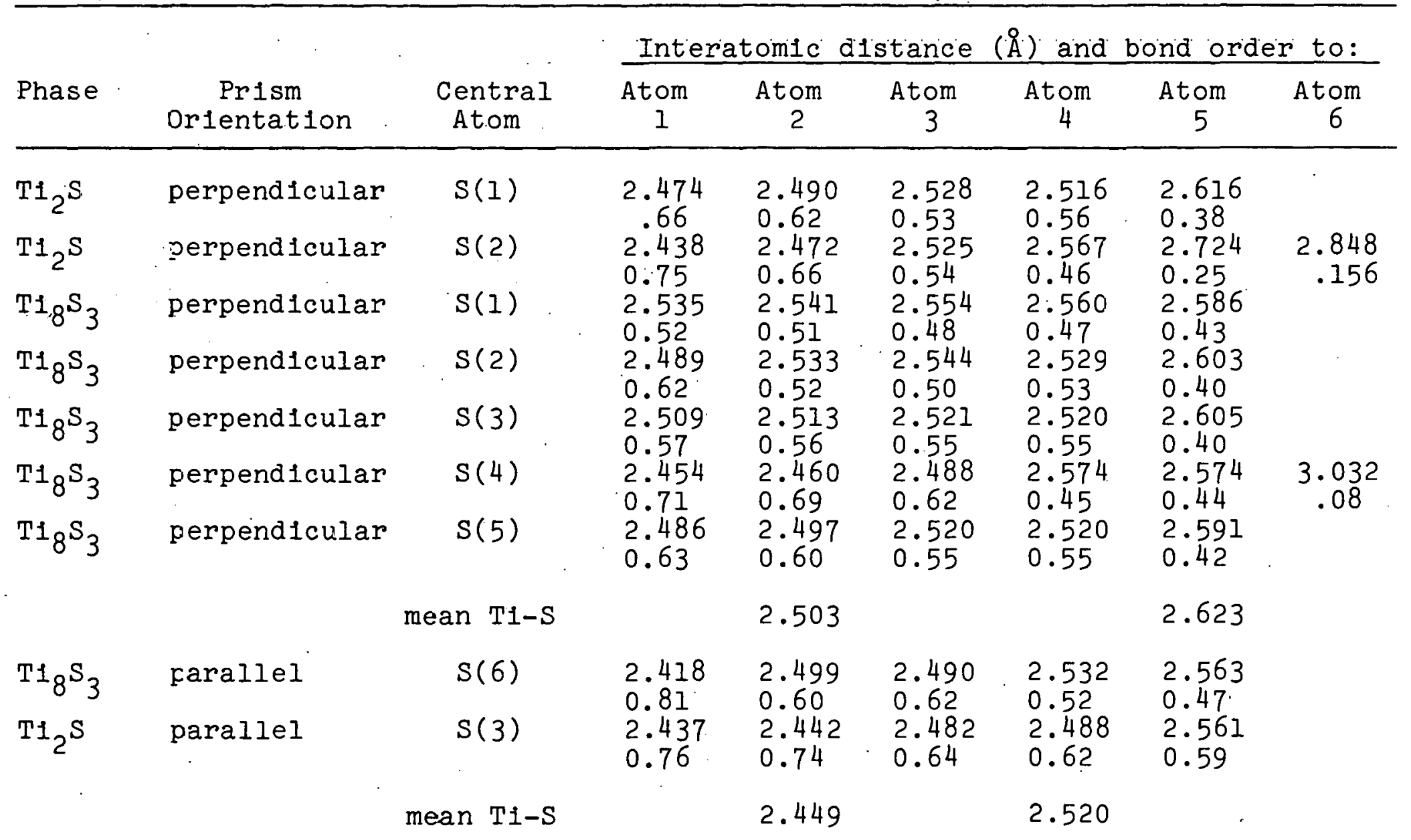




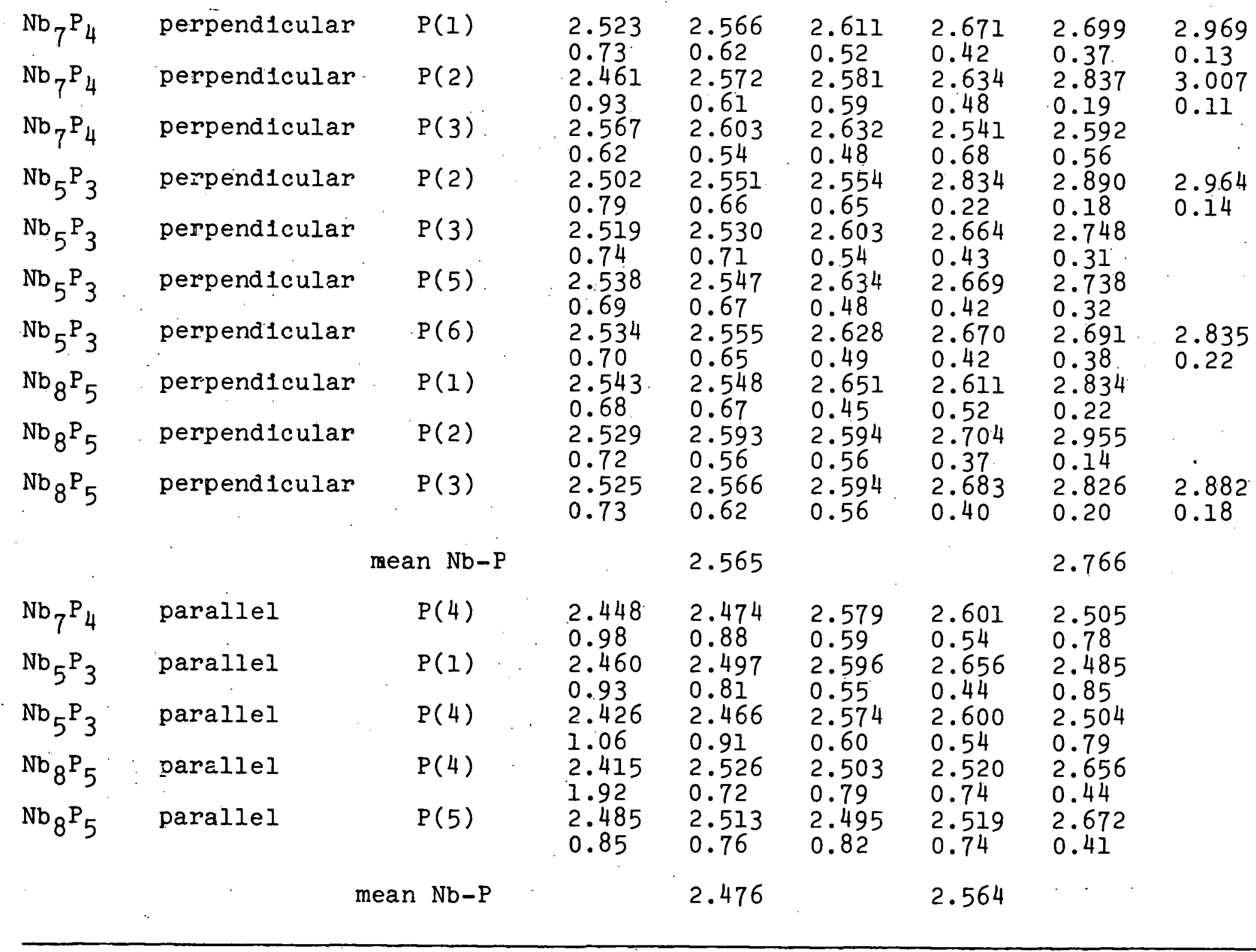


forming the prism. This suggests that perhaps only the distances to the six atoms forming the trigonal prism should be compared between the prisms of different orlentation. Because the interatomic distances vary over a large range, it is difficult to determine if there are any observable trends based on the differences in interatomic distances. For example, the distances to the capping atoms of the perpendicular prism in the $\mathrm{Nb}-\mathrm{P}$ system yleld the observation that one of the interatomic distances is noticeably larger for most of the nonmetal atoms. Even though this generalization may have some validity, there are always exceptions that occur, e.g., $\mathrm{P}(3)$ of $\mathrm{Nb}_{7} \mathrm{P}_{4}$, which cast doubt on any general conclusion.

One trend, however, that does seem significant concerns a possible difference in interatomic distances for the parallel orientation of the trigonal prism. If the distances to atoms 1 and 2 in the parallel-axis prism are compared with the distances to atoms 3,4 , and 5 of the same prism or atoms 1, 2, and 3 of the perpendicular-axis prism, there is a strong indication that the distances to atoms 1 and 2 of the parallel-axis prism are significantly shorter. It is the difference in mean values and the greater varlation for $\mathrm{X}-\mathrm{Me}$ distances to atoms 3,4 , and 5 that combine to suggest a difference in the nature of the nonmetal bonding contribution to the different metal atoms forming the parallel-axis trigonal prism. 
Since the variation in bond length is an integral feature of the coordination polyhedra, these polyhedra are In fact less regular in geometry than may have been suggested by the original description. In the qualitative bonding description that follows, the polyhedra will be treated as if their geometry were regular as a first approximation, and an attempt will be made to justify, in a qualitative way, the variations in interatomic distances.

\section{Nonmetal Capping Atoms for P.C.P. II and IV}

A comparison of interatomic distances for certain P.C.P. in different metal-nonmetal systems provides valuable information concerning differences in chemical interaction. One question often encountered in studying structures of the layered class concerns the degree of bonding Interaction between a central metal atom in a given P.C.P. and those atoms which are capping atoms located off the faces of the coordination polyhedra. It is the point of view adopted here that the relative degree of bonding interaction to such capping atoms can be qualitatively evaluated by comparing the interatomic distances to the capping atoms w1th the distances to the same type of atom which are part of the P.C.P.

Figure 9 above 1llustrates the metal P.C.P. II and IV. The two polyhedral faces of unit IV formed by atoms 2 and 3 
and by atoms 3 and 4 often have a nonmetal capping atom in the structures of the layered class. Similarly, unit II in this figure often has a nonmetal capping atom off the face formed by atoms 4 and 5. Since both P.C.P. IV and II also contain nonmetal atoms as part of the P.C.P. (atom 1 for unit IV and atoms $I$ and 2 for unit II), there is a question whether the nonmetal capping atoms should also be considered as part of the P.C.P. of the central atom.

Table 13 compares the interatomic distances to the capping atoms with the interatomic distances to the nonmetal atoms which are part of the P.C.P. The values listed for the $\mathrm{Ti}-\mathrm{S}$ and $\mathrm{Nb}-\mathrm{P}$ systems are the mean interatomic distances for the $\mathrm{Ti}_{2} \mathrm{~S}, \mathrm{Ti}_{8} \mathrm{~S}, \mathrm{Nb}_{7} \mathrm{P}_{4}, \mathrm{Nb}_{5} \mathrm{P}_{3}$ and $\mathrm{Nb}_{8} \mathrm{P}_{5}$ phases. It is the large difference in interatomic distances to capping nonmetal atoms relative to the nonmetal atoms forming the P.C.P. which suggests that the bonding interactions to the cappling nonmetal atoms can be ignored in a crude first approximation approach to the consideration of bonding in the Ti-S and Nb-P systems.

In contrast, the relative interatomic distances to the nonmetal capping atoms for P.C.P. II and IV in the $\mathrm{Fe}_{2} \mathrm{P}$ and $\mathrm{Co}_{2} \mathrm{P}$ structures are actually. equal to or less than the distances to nonmetal atoms In the P.C.P. The implication is that for $\mathrm{Fe}_{2} \mathrm{P}$ and $\mathrm{Co}_{2} \mathrm{P}$ the bonding interaction to the capping atioms is actually equal in strength to.or stronger 
than the interaction with nonmetal atoms forming the P.C.P. This difference also suggests a difference in the nature of the metal bonding contribution of $\mathrm{Fe}$ and $\mathrm{Co}$ when compared to the bonding contribution of $\mathrm{Ti}$ or $\mathrm{Nb}$.

Table 13. Relative interatomic distances to polyhedral and cappling nonmetal atoms of. P..C..P.. II and IV.

\begin{tabular}{|c|c|c|c|}
\hline \multirow[b]{2}{*}{ Sys.tem/Phase. } & \multicolumn{3}{|c|}{ Interatomlic Distances $(\mathrm{Me}-\mathrm{X})$} \\
\hline & Polyhedral $\mathrm{X}$ & Capplng $\mathrm{X}$ & P..C.P. \\
\hline$T 1-S$ & 2.508 & 2.940 & II \\
\hline T1-S & 2.462 & 2.544 & IV \\
\hline $\mathrm{Nb}-\mathrm{P}$ & 2.572 & 2.841 & II \\
\hline $\mathrm{Nb}-\mathrm{P}$ & 2.482 & 2.599 & IV \\
\hline $\mathrm{Fe}_{2} \mathrm{P}$ & 2.484 & 2.381 & II \\
\hline $\mathrm{Fe}_{2} \mathrm{P}$ & 2.484 & & II \\
\hline $\mathrm{Fe}_{2} \mathrm{P}$ & 2.289 & 2.219 & IV \\
\hline $\mathrm{Fe}_{2} \mathrm{P}$ & & 2.219 & IV \\
\hline $\mathrm{CO}_{2} \mathrm{P}$ & 2.399 & 2.294 & II \\
\hline $\mathrm{Co}_{2} \mathrm{P}$ & 2.543 & & II \\
\hline $\mathrm{Co}_{2} \mathrm{P}$ & 2.233 & 2.143 & IV \\
\hline $\mathrm{Co}_{2} \mathrm{P}$ & & 2.238 & IV \\
\hline
\end{tabular}




\section{Differences in Me-X Bond Distances for Units ViI and VII}

A survey of the various structures in the layered class Indicates that there is a close relationship between the presence of a trigonal prism with parallel axis and the metal coordination polyhedra of type VI and VII. The relationship between these coordination polyhedra is 1llustrated in Figure 11. Four coordination polyhedra formed from twelve atoms are represented in this drawing, and the metal atoms important to the following discussion are numbered, while the nonmetal atoms are represented by a letter. The two trigonal prisms about atoms $a$ and $b$ are outlined by solid Iines. The prism about atom $b$ shares an edge with each of two prisms about atom a. Broken lines represent the coordination polyhedra about metal atoms 1 and 2. Position $c$ of the metal polyhedra can be elther a metal or a nonmetal atom; a metal atom at position $c$ implies that unit VII Is the coordination polyhedra of atom 1 or 2 , while a nonmetal at position c corresponds to a coordination polyhedra of. type VI.

The particular arrangement of atoms depicted in this drawing occurs for all of the structures of this class which have a trigonal prism with axis parallel to the two planes of atoms (certain $\mathrm{Me}_{4} \mathrm{X}_{3}$ structure types contain an additional feature). The following chapter (VI) considers the possible 


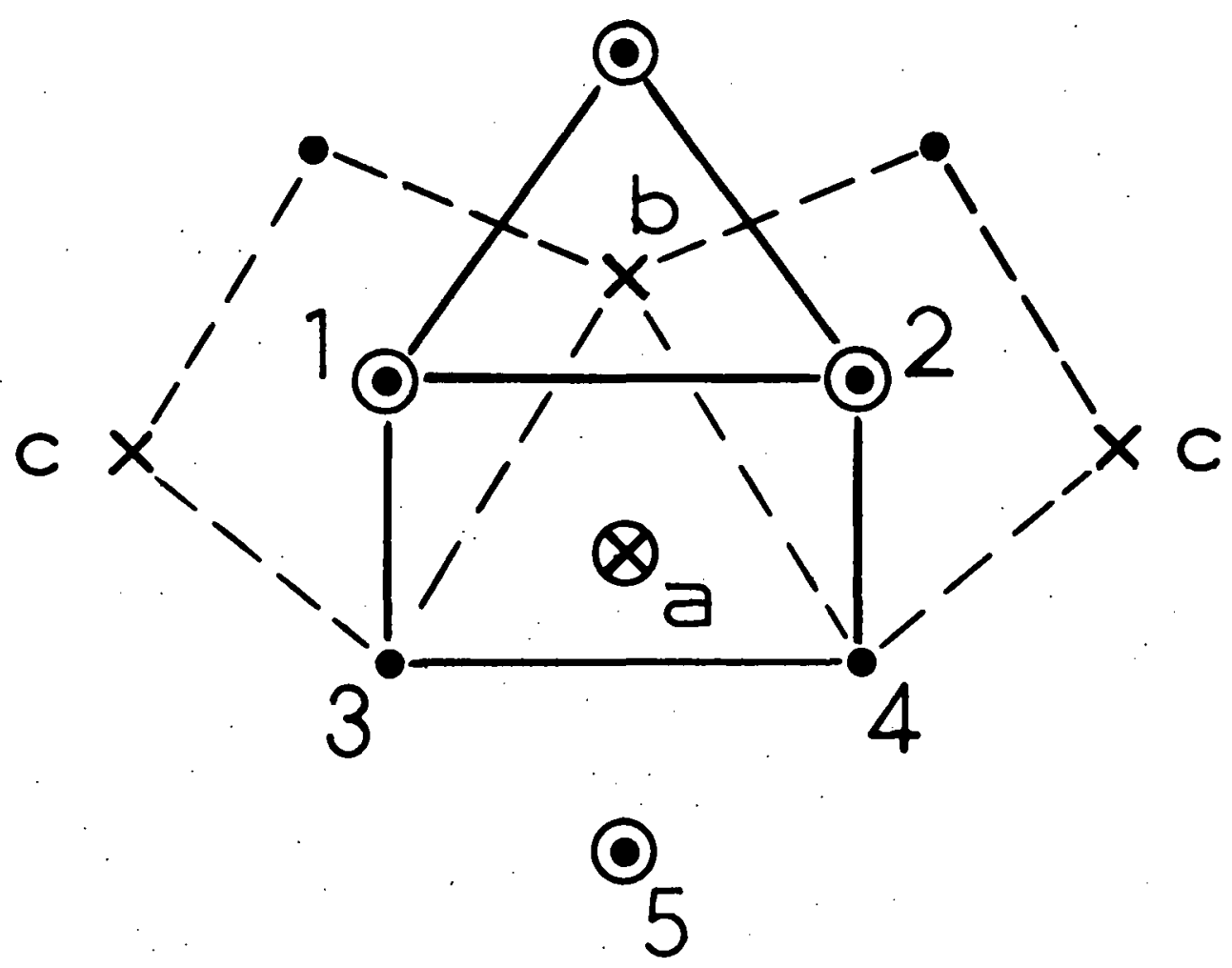

Figure 11. A structural unit as a combination of metal and nonmetal P.C.P. 
use of this unit in helping to solve an unknown structure for a phase that is expected to be a member of this layered class.

The numbers and letters in the column headings for columns 3, 6, 7, and 8 in Table 14 correspond to the atom designations in Figure 11. The first two columns indicate the compound and particular nonmetal atom of that compound with the parallel trigonal prism as its partial coordination polyhedron. Columns 3,4 , and 5 identify the two metal atoms and their type of P.C.P. which correspond to metal atoms 1 and 2 in Figure 11 . Interatomic distances from each of these metal atoms to the various nonmetal atoms of their coordination polyhedra are given in the last three columns of the table. As a specific example, consider the first two rows of the table. For $\mathrm{TI}_{2} \mathrm{~S}, \mathrm{~S}(3)$ corresponds to atom a whlle $T 1(6)$ and $T 1(5)$ correspond to atoms $I$ and 2, respectively, in Figure 11. The three interatomic distances associated with $\mathrm{T}_{1}(6)$ are the distances from $\mathrm{TI}(6)$ to the nonmetal atom positions of 1ts partial coordination. polyhedron. Since the P.C.P. of $T_{1}(6)$ is unit VII, which has only two independent nonmetal atom positions, the entry is made in the last column.

If a comparison is made of the interatomic distances listed in the column represented by atom $\underline{a}$ with the distances in columns $b$ and $c$, the entries in column a indicate significantly shorter boni distances. At the botlum of the table 
the average values of each of the Me-X interatomic distances are listed separately for the T1-S and $\mathrm{Nb}-\mathrm{P}$ systems. The difference in bond distances indicated by this table is large enough to suggest that there may be a difference in the bonding contribution from the central metal atom of P.C.P. VI and VII to the different nonmetal atoms in the coordination polyhedron. The qualitative bonding considerations which follow will of fer a possible explanation for this difference in bond length. 
Table 14. Differences in Me-X interatomic distances for P..C.P.. VI. and VII

\begin{tabular}{|c|c|c|c|c|c|c|c|}
\hline \multirow[b]{2}{*}{ Phase. } & \multirow{2}{*}{$\begin{array}{l}\text { Parallel } \\
\text { Prism }\end{array}$} & \multirow{2}{*}{$\begin{array}{l}\text { Metal } \\
\text { Atom }\end{array}$} & \multirow{2}{*}{$\begin{array}{l}\text { Atom } \\
\text { No.. }\end{array}$} & \multirow{2}{*}{$\begin{array}{c}\text { Type } \\
\text { P.C..P.. }\end{array}$} & \multicolumn{3}{|c|}{ Interatomic Distance to } \\
\hline & & & & & A.tom a & A.tom b. & Atom c \\
\hline $\mathrm{TI}_{2} \mathrm{~S}$ & $S(3)$ & $\frac{1}{2}$ & $\begin{array}{l}\mathrm{Ti}(6) \\
\mathrm{T} 1(5)\end{array}$ & $\begin{array}{l}\text { VII } \\
\text { VI }\end{array}$ & $\begin{array}{l}2.437 \\
2.442\end{array}$ & $\begin{array}{l}2.490 \\
2.528\end{array}$ & $\begin{array}{l}\text { Meta } 1 \\
2.472\end{array}$ \\
\hline \multirow[t]{2}{*}{$\mathrm{Ti}_{8} \mathrm{~S}_{3}$} & $S(6)$ & $\frac{\pi}{2}$ & $\begin{array}{l}\mathrm{T} 1(13) \\
\mathrm{T} 1(15)\end{array}$ & $\begin{array}{l}\text { VII } \\
\text { VII }\end{array}$ & $\begin{array}{l}2.418 \\
2.499\end{array}$ & $\begin{array}{l}2.541 \\
2.554\end{array}$ & $\begin{array}{l}\text { Metal } \\
\text { Metal }\end{array}$ \\
\hline & & & & Mean & 2.449 & \multicolumn{2}{|c|}{2.517} \\
\hline $\mathrm{Nb}_{7} \mathrm{P}_{4}$ & $P(4)$ & $\frac{1}{2}$ & $\begin{array}{l}\mathrm{Nb}(5) \\
\mathrm{Nb}(7)\end{array}$ & $\begin{array}{l}\text { VI } \\
\text { VI }\end{array}$ & $\begin{array}{l}2.448 \\
2.474\end{array}$ & $\begin{array}{l}2.603 \\
2.567\end{array}$ & $\begin{array}{l}2.523 \\
2.572\end{array}$ \\
\hline $\mathrm{Nb}_{8} \mathrm{P}_{5}$ & $P(4)$ & $\begin{array}{l}1 \\
2\end{array}$ & $\begin{array}{l}\mathrm{Nb}(7) \\
\mathrm{Nb}(6)\end{array}$ & $\begin{array}{l}\mathrm{VI}^{\mathrm{a}} \\
\mathrm{VI}\end{array}$ & $\begin{array}{l}2.526 \\
2.465\end{array}$ & $\begin{array}{l}2.548 \\
2.651\end{array}$ & $\begin{array}{l}2.529 \\
2.594\end{array}$ \\
\hline $\mathrm{Nb}_{8} \mathrm{~F}_{5}$ & $P(5)$ & $\frac{1}{2}$ & $\begin{array}{l}\mathrm{Nb}(9) \\
\mathrm{Nb}(8)\end{array}$ & $\begin{array}{l}\text { VI } \\
\text { VII }\end{array}$ & $\begin{array}{l}2.513 \\
2.485\end{array}$ & $\begin{array}{l}2.593 \\
2.594\end{array}$ & $\begin{array}{l}2.525 \\
\text { Metal }\end{array}$ \\
\hline $\mathrm{Nb}_{5} \mathrm{P}_{3}$ & $P(1)$ & $\begin{array}{l}1 \\
2\end{array}$ & $\begin{array}{l}\mathrm{Nb}(3) \\
\mathrm{Nb}(\mathrm{I})\end{array}$ & $\begin{array}{l}\text { VII } \\
\text { VI }\end{array}$ & $\begin{array}{l}2.497 \\
2.460\end{array}$ & $\begin{array}{l}2.538 \\
2.634\end{array}$ & $\begin{array}{l}\text { Metal } \\
2.534\end{array}$ \\
\hline \multirow[t]{2}{*}{$\mathrm{Nb}_{5} \mathrm{P}_{3}$} & $P(4)$ & $\begin{array}{l}1 \\
2\end{array}$ & $\begin{array}{l}\mathrm{Nb}(4) \\
\mathrm{Nb}(7)\end{array}$ & $\begin{array}{l}\text { VI } \\
\text { VI }\end{array}$ & $\begin{array}{l}2.426 \\
2.466\end{array}$ & $\begin{array}{l}2.603 \\
2.519\end{array}$ & $\begin{array}{l}2.547 \\
2.554\end{array}$ \\
\hline & & & & Mean & 2.476 & \multicolumn{2}{|c|}{2.568} \\
\hline
\end{tabular}

$a_{\mathrm{Nb}}(7)$ atom position is only partially filied. 
VI. STRUCTURAL IMPLICATIONS OF THE PARALLEL TRIGONAL PRISM

At the end of the last chapter, a larger structural unit was described in terms of a specific combination of two metal and two nonmetal coordination polyhedra. A structural unit of this type occurs in 14 of the 19 structure types in the layered class. The arrangement of atoms in this unit suggests certain limitations on the types of P.C.P. that can be linked with this unit. If the presence of this unit can be assumed for any unknown structure of the layered class, it can be used as a guide in structure solution. The five remaining structure types of this class $\left(\mathrm{Fe}_{2} \mathrm{P}, \mathrm{Co}_{2} \mathrm{P}, \mathrm{Fe}_{2} \mathrm{As}, \mathrm{V}_{12} \mathrm{P}_{7}, \mathrm{Rh}_{4} \mathrm{P}_{3}\right)$ have structural features which are surprisingly similar, but which distinguish them from the other members of the layered class.

\section{A. Structural Limitations}

Figure 11 above 1llustrates a structural unit formed by four P.C.P. for which the polyhedra of atoms 1 and 2 could be of either type VI or VII. The possible combinations of P.C.P. for atoms 1 and 2 Indicate that there are only three different structural units formed by the four P.C.P. The case where both 1 and 2 have P.C.P. of type VII will be represented by the symbolism VII-1l-prismVII, while the case where both atoms have P.C.P. VI w1ll be 
represented by VI-(II)prism-VI. If atom $I$ and atom 2 have different coordination polyhedra, the unit will be represented by VI-(II)prism-VII.

For each of these three cases the arrangement of atoms provides a severe limitation on the possible coordination polyhedra of atoms 3 and 4 in Figure 11 . The coordination polyhedra of atom 3 must contain the atoms designated as 1 , a, and 5 in the figure. Of the eight metal coordination polyhedra, only units II, III, and VI have an arrangement of atoms simflar to atoms 1 , a, and 5, indicating that the partial coordination polyhedron of atom 3 will have to be II, III, or VI. Similar considerations apply to atom 2, indicating it also will have elther unit II, III, or VI as its P.C.P. Thus; the arrangement of atoms forming the structural unit decreases the number of possible P.C.P. for atoms 3 and 4 from eight to three.

Figure 12 (a through f) 1llustrates some of the possible orientations of units II and VI (for atoms 3 and 4) for the particular case VI-(II)prism-VI. Combining all three cases and lgnoring any other structural limitations, there are 45 distinct orientations of P.C.P. for atoms 3 and 4. Figure 12(0) illustrates one of the 45 possible units, but it also illustrates a second structural limitation inherent in the nature of the structures of the layered class. At the left side of this figure the 
Figure 12. Examples of some of the different possible combinations of metal P.C.P. for atoms 3 and 4 in the VI-(II)prism-VI structural unit. The orlentation of P.C.P. In part c) and $d$ ) of the figure have not been observed for structures in the layered class 


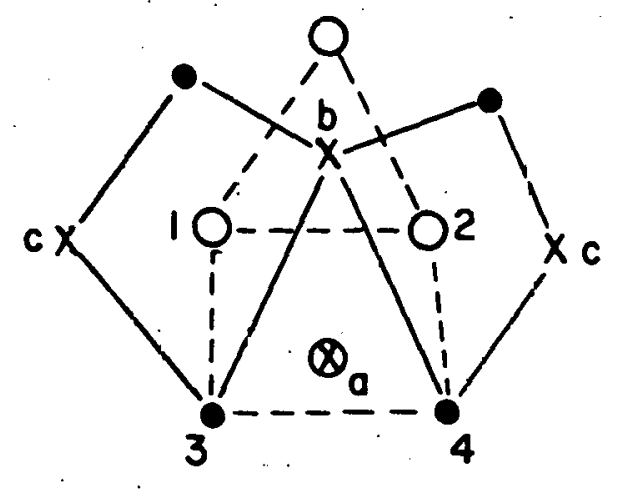

a)

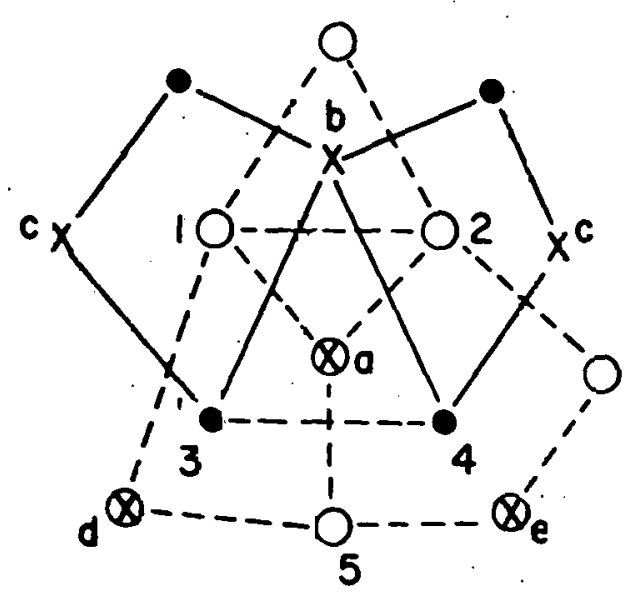

d)

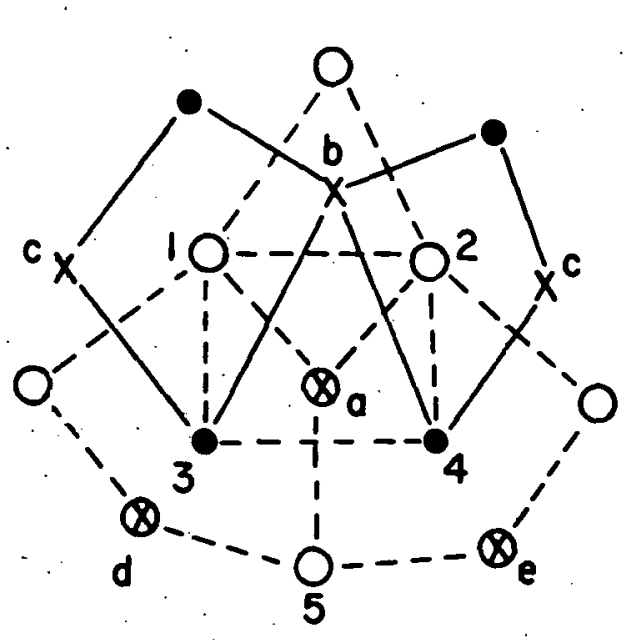

b)

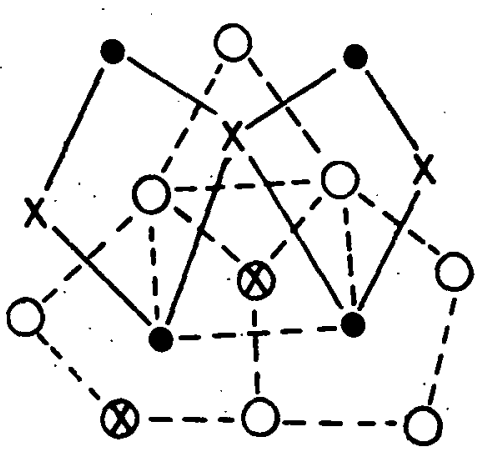

e)

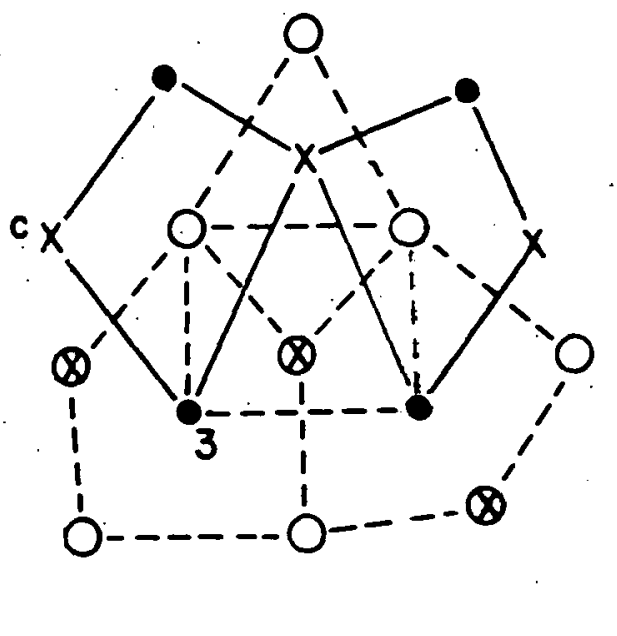

c)

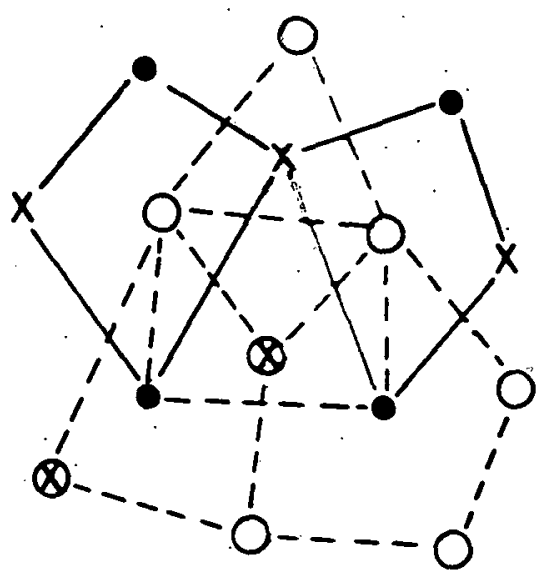

f)

吕 
orientation of unit II for atom 3 would place a nonmetal of unit II in close proximity of the nonmetal at atom c. The close proximity of these two nonmetal atoms implies that each will be part of the coordination polyhedron of the other nonmetal, thus implying that nelther would have a trigonal prismatic partial coordination polyhedron since such $X-X$ bonds are incompatible with the observed trigonal prismatic coordination in the structures of this class. Since the orientation of P.C.P. II in the unit of Figure 12 (c) implies $X-X$ bonds, it can be eliminated as a possible un1t. Although similar considerations applied to the other possible units reduce the number of possibilities from 45 to 33 , the real significance of the treatment is the 1llustration of the restrictive influence implied by the requirement that nonmetal atoms have trigonal prismatic P.C.P, on the nature of the P.C.P. for atoms near the four nonmetal atoms in these units.

As a further illustration, consider the unit pictured In Figure $12(d)$ and the possible coordination polyhedra for atom 5. Atoms 3 and 4 indicate that the coordination polyhedron of atom 5 will have to contain a face formed by four metal atoms so that units VI and VIII can be eliminated as posstble P.C.P. for atom 5. The positions of the two nonmetal atoms $\underline{d}$ and $\underline{e}$ Indicate that there cannot be a nonmetal atom as part of the coordination polyhedron of atom 5 which w1ll be close to efther of the two faces represented by the 
two pairs of atoms $\underline{d}$ and 5 and $\underline{e}$ and 5 . A nonmetal close to either of these faces would require that the polyhedron for nonmetal atoms $\underline{d}$ or $\underline{e}$ is not trigonal prismatic. These considerations eliminate all but units I, III, and VII as possible P.C.P. for atom 5 .

\section{B. A Possible Method for structural Solution}

The recurrence of the large structural unit described above for 14 of the 19 structure types of the layered class suggests that it may be of use in the solution of an unknown crystal structure of any new phase that might be expected to be a member of this layered structural class. All three of the units considered above are formed from twelve atoms, indicating that the units have appreciable size relative to the dimensions of the unit cells in which they have been observed. For many of these structures the structural unit accounts for a large percentage of the independent atom positions in the appropriate structure.

Although the $\mathrm{Me}_{4} \mathrm{X}_{3}$ phases were excluded in the earlier discussion of this structural unit, all but the $\mathrm{Rh}_{4} \mathrm{P}_{3}$ phase are included in the 14 phases containing this structural unit. The $\mathrm{Me}_{4} \mathrm{X}_{3}$ structure types form a special case due to the presence of the metal partial coordination polyhedron of type VIII for metal atoms 1 and 2 (Figure 11 ). The utility of the structural unit in structure solution is applicable whether or not F.C.P. VIII is considered, but 
unless the sto1chlometry is known to be $\mathrm{Me}_{4} \mathrm{X}_{3}$, unlt VIII need not be directly considered. The $\mathrm{Nb}_{5} \mathrm{Se}_{4}$ and $\mathrm{Nb}_{2} \mathrm{Se}$ phases are included in this group of structures even though their trigonal prisms are incomplete, because they are of the correct orlentation and contain units VI $\left(\mathrm{Nb}_{5} \mathrm{Se}_{4}\right)$ and VII $\left(\mathrm{Nb}_{2} \mathrm{Se}\right)$.

In attempting to solve an unknown crystal structure of the type under consideration, the structural chemist usually has available detalled knowledge of the space group, lattice parameters, and stoichiometry of the phase. All of this Information is useful in limiting the structural units used. and in determining the orientation of a particular unit within the unit cell. As an example of the ut1lity of this approach, assume that $\mathrm{TI}_{2} \mathrm{~S}$ contains a general structural unit (1..e., the cholce of P.C.P. for atoms 1 and 2 have not been specified as VI or VII), assume that only the lattice parameters, space group, density, and sto1chiometry of $\mathrm{Ti}_{2} \mathrm{~S}$ are known, and then consider the number of trial structures that are consistent with the packing of the general structural unit under the limitations of the known crystallographic data.

Full appreclation of this technique requires the use of two dimensional models for the general structural unit, but the main considerations underlying this approach can be illustrated in the following example: 
1) The experimental density of $\mathrm{TI}_{2} \mathrm{~S}$ indicated twelve $\mathrm{TI}_{2} \mathrm{~S}$ units or 36 atoms in the unit cell.

11) The structural units under consideration all contain twelve atoms.

iii) Early in the structure determination of $\mathrm{Ti}_{2} \mathrm{~S}$, it was suggested that the occupied atom positions corresponded to the $4(\mathrm{~g})$ positions of the Pnnm space group, indicating that the structural unit would have to be repeated four times within the unit cell. Four-fold repetition of the general structural unit would imply 48 atoms in the unit cell in contradiction to the 32 atoms indicated experimentally unless some atoms of one structural unit were also common to neighboring units.

iv) Figure 13a) illustrates the inversion centers and n-glide planes present in the Pnnm space group of $\mathrm{TI}_{2} \mathrm{~S}$. The dimensions of the drawing reflect the ratio of the known lattice parameters. Since the structural unit does not contain an inversion center, each of the four units (one structural unit repeated three times) would have to be falrly well-confined to one of the four rectangular subdivisions of the unit cell formed by the inversion centers.

v) The n-glide planes of the space group provide severe IImitations on the orientation of the assumed structural unit within each of the rectangular regions. The nature of the Iimitations can be seen by considering the 
orientation of the structural unit in the actual $\mathrm{TI}_{2} \mathrm{~S}$ structure, Figure 13b), and trying to change the orlentation of the structural unit silghtly as represented in Figure 13c). Comparison of the unit in the upper left corner of Figure 13b) w1th the un1t in the left corner of F1gure 13c) shows that they differ only slightly in orlentation. The right half of part $c$ ) indicates a second unit related to the first by the n-glide plane represented in the figure. The numbers ldentify atoms in the two units which would have interatomic distances implying repulsive interaction between each pair of atoms. Any attempt to reduce the repulsive interactions by translating one unit relative to the second in a direction parallel to the a-axis would imply an increase in the magnitude of the a-axis. (A translation with a component parallel to the b-axis is not possible because of the n-glide plane.) The same type of considerations can be applied to the unit in the lower left region of the unit cell with similar results. Work with simple models suggests that the orientation of units in Figure 13b) is the only possible orientation consistent with the symmetry and lattice parameters.

vi) In the considerations so far there has been no reference to the known stolchiometry of a spec1f1c structural un1t. The actual structural un1t was represented in Figure 13b), and any other cholce of a structural unft 


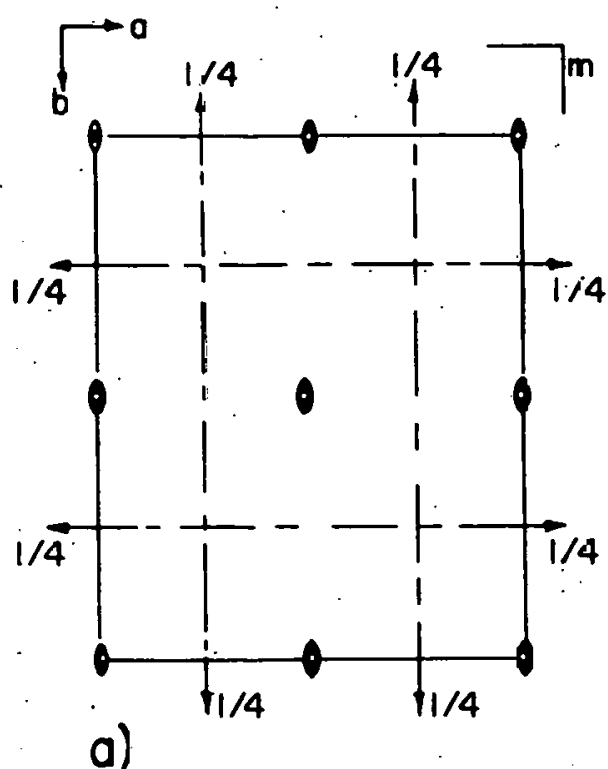

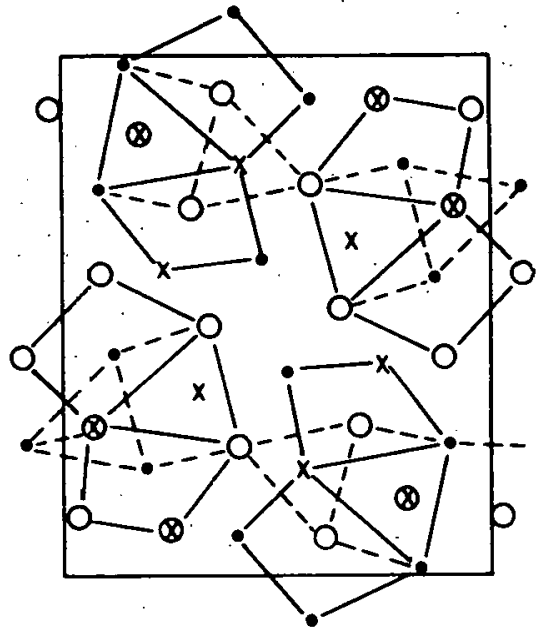

b)

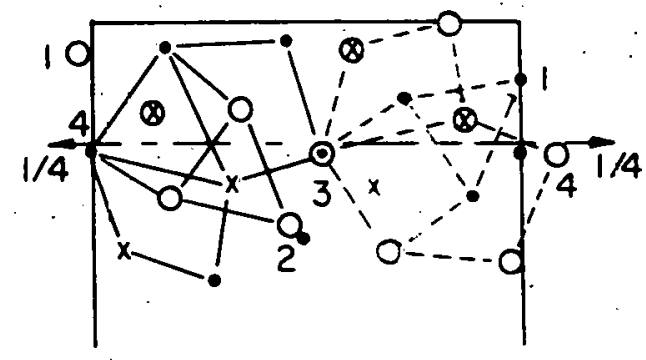

占

c)

a) illustrates the symmetry operations of the Pnnm space group of $\mathrm{TI}_{2} \mathrm{~S}$

b) illustrates the actual orientation of the structural unit in $\mathrm{Ti}_{2} \mathrm{~S}$

c) shows the effect of a slight movement of the unit from the observed orientation

Figure 13. The occurrence of the VII-(II)prism-VI structural unit in the $\mathrm{TI}_{2} \mathrm{~S}$ structure 
(VI-(II)prIsm-VI or VII-(II)prism-VII) would not correspond to the experimentally determined stolchiometry.

$$
\begin{gathered}
\text { C. The } \mathrm{Fe}_{2} \mathrm{P}, \mathrm{Co}_{2} \mathrm{P}, \mathrm{Fe}_{2} \mathrm{As}, \mathrm{V}_{12} \mathrm{P}_{7} \text {, and } \\
\mathrm{Rh}_{4} \mathrm{P}_{3} \text { Structure Types }
\end{gathered}
$$

The arbitrary separation of the structure types of the layered class into two different groups presented in the last section was based in part on whether or not the structures contained a trigonal prism with axis parallel to the planes of atoms forming the structure. The division of the structure types based on the presence of a parallel trigonal prism is certainly not exclusive. For example, the $\mathrm{Rh}_{4} \mathrm{P}_{3}$ structure type contains $P(3)$ with a parallel trigonal prism, yet this phase has structural similarities to $\mathrm{Fe}_{2} \mathrm{P}, \mathrm{Co}_{2} \mathrm{P}$, $\mathrm{Fe}_{2} \mathrm{As}$, and $\mathrm{V}_{12} \mathrm{P}_{7}$, none of which contain a trigonal prism with axis parallel to the layered atom positions. Similarly, the grouping of the three trigonal prisms about $S(2), S(3)$, and $S(5)$ with perpendicular axes in $\mathrm{TI}_{8} \mathrm{~S}_{3}$ has structural features similar to $\mathrm{Fe}_{2} \mathrm{P}, \mathrm{Co}_{2} \mathrm{P}$, etc., but overall the structural features of $\mathrm{TI}_{8} \mathrm{~S}_{3}$ correspond more closely with the structural features of the remaining phases of the layered structural class.

The structural similarities of the $\mathrm{Fe}_{2} \mathrm{P}, \mathrm{Co}_{2} \mathrm{P}, \mathrm{Fe}_{2} \mathrm{As}$, $\mathrm{V}_{12} \mathrm{P}_{7}$, and $\mathrm{Rh}_{4} \mathrm{P}_{3}$ structures can best be seen by considering a projection of the two layers of atom positions that form the structures. In profection, the nonmetal atoms form the 
regular hexagonal rings illustrated in Figure 14. It should be noted that the hexagonal rings in this drawing do not represent closely packed layers of nonmetal atoms, since the various atoms are not all in the same layer. The difference in the layering of atoms for each hexagonal ring is indicated in the figure.

Each of the hexagonal rings formed by the projection of the atoms can be divided into six triangular segments. In projection the metal atom positions of each structure all. fall within the triangular segments. In each of the structures represented in Figure 14, a metal atom projected into one triangular section is always from a different layer of the structure than the metal atoms projected into the two adjacent triangular segments.

It is interesting that the hexagonal network formed by the projection of the nonmetal atoms very closely approximates a network formed by regular hexagons. This regularity is even more striking when one considers the low space group symmetry for the $\mathrm{Co}_{2} \mathrm{P}, \mathrm{Rh}_{4} \mathrm{P}_{3}, \mathrm{Fe}_{2} \mathrm{As}$, and $\mathrm{V}_{12} \mathrm{P}_{7}$ structures. It is the presence of the regular hexagonal networks which emphasizes the similarity between these different structure types. In contrast, the projected metal atom positions do not fall at the center of the triangular segments but rather exhibit variations in the different structure types. 
Figure 14. Hexagonal rings formed by the projections of nonmetal atom positions in the two layers of each structure 

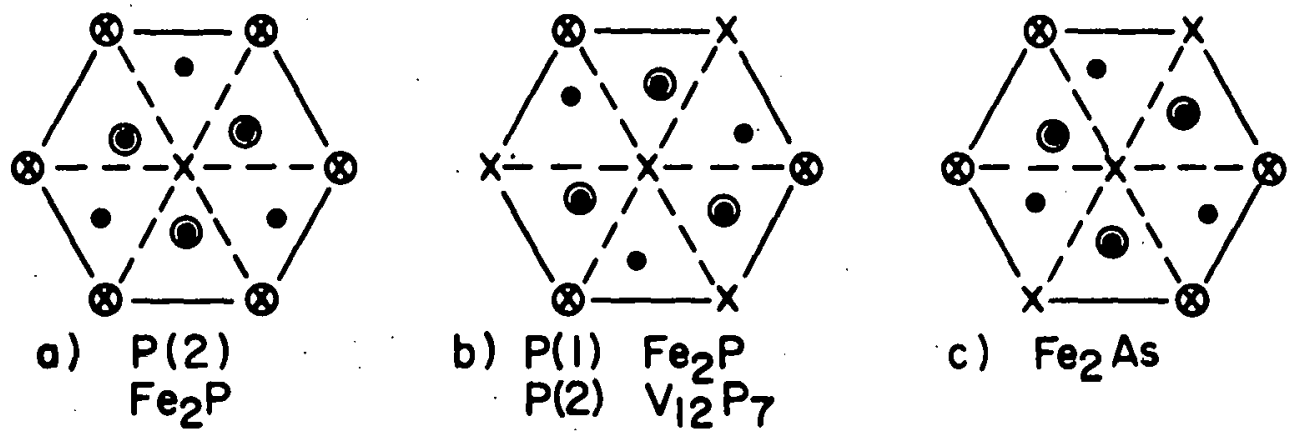

b) $\begin{array}{ll}P(1) & F_{2} P \\ P(2) & V_{12} P_{7}\end{array}$

c) $\mathrm{Fe}_{2} \mathrm{As}$
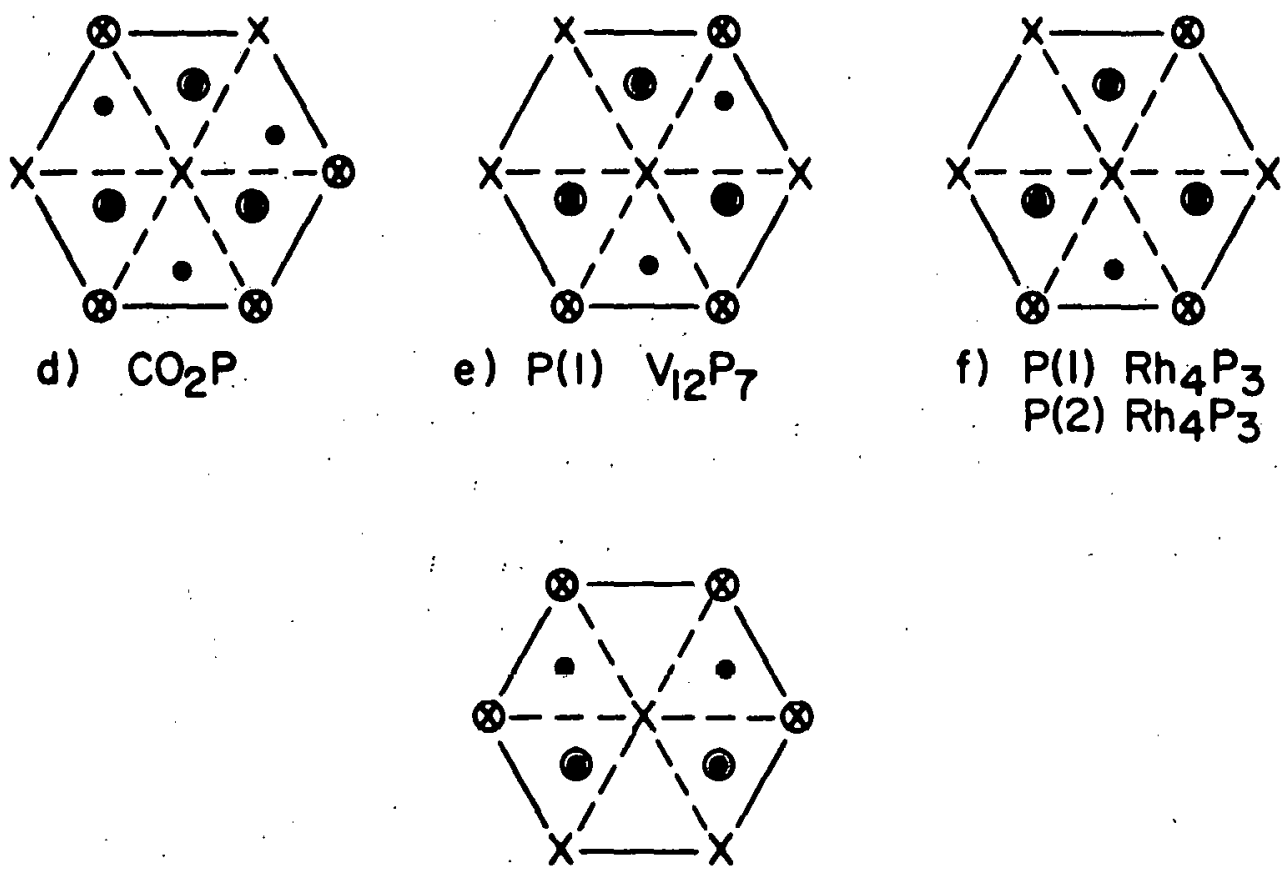

g) $P(3) R h_{4} P_{3}$ 
The varlation of the projected metal atom positions within the triangular segments is due in part to atom packing considerations. For example, the hexagonal rings In Figure 14e), f), and $g$ ) each contain a triangular segment formed by a projection of three nonmetal atoms whlch are located in the same layer. Any attempt to place a metal atom within these triangular segments would cause repulsive interaction between the metal atom and at least one of the three nonmetal atoms. The three triangular segments of this type are all empty for the phases where they are observed. The occurrence of other triangular segments that are also empty, even though the nonmetal atoms defining the triangular segment are not all in the same layer, indicate that it is not solely packing considerations that determine the presence or absence of a metal atom in each segment.

Figure 15a) and b) illustrate the $\mathrm{Fe}_{2} \mathrm{P}$ and $\mathrm{Co}_{2} \mathrm{P}$ structures, emphasizing the presence of these hexagonal rings. Each of the structure types under considerations here can be described in terms of a different packing arrangement of the various hexagonal rings. The underlying reasons for the occurrence of different units for the different phases is not understood and will not be pursued at this time. 
a. $\mathrm{Fe}_{2} \mathrm{P}$

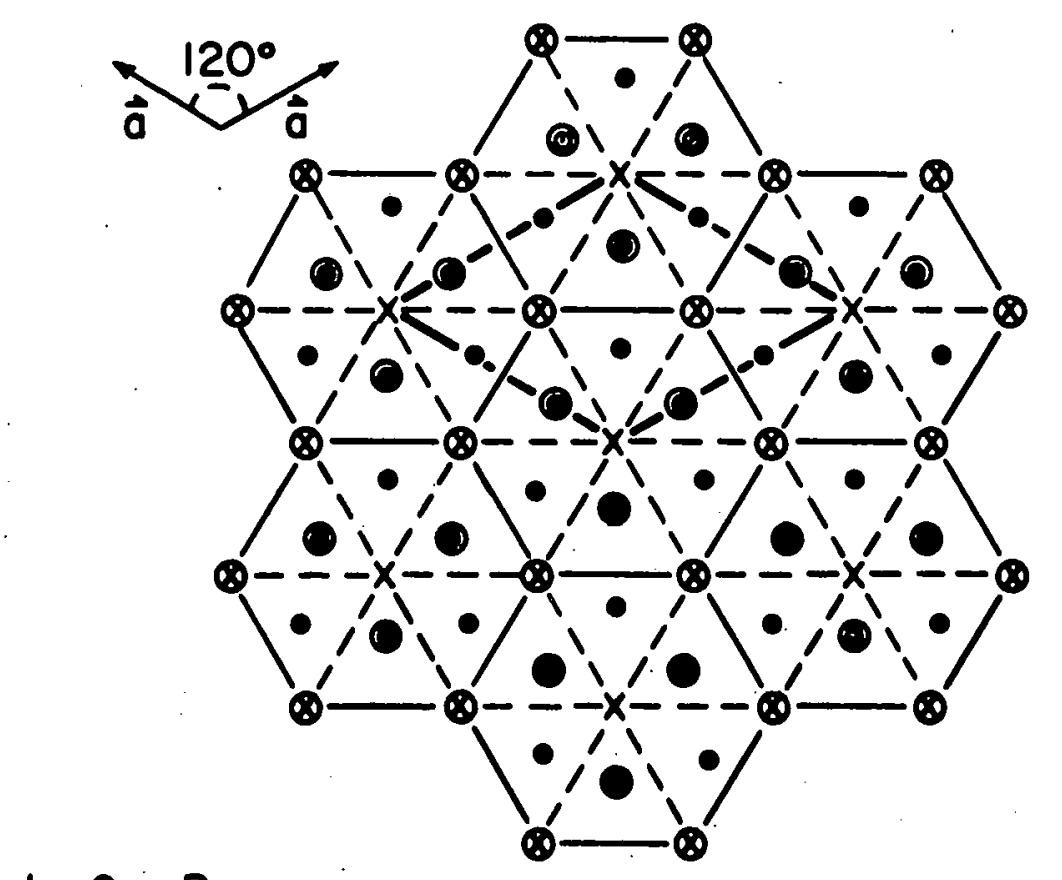

b. $\mathrm{Co}_{2} \mathrm{P}$

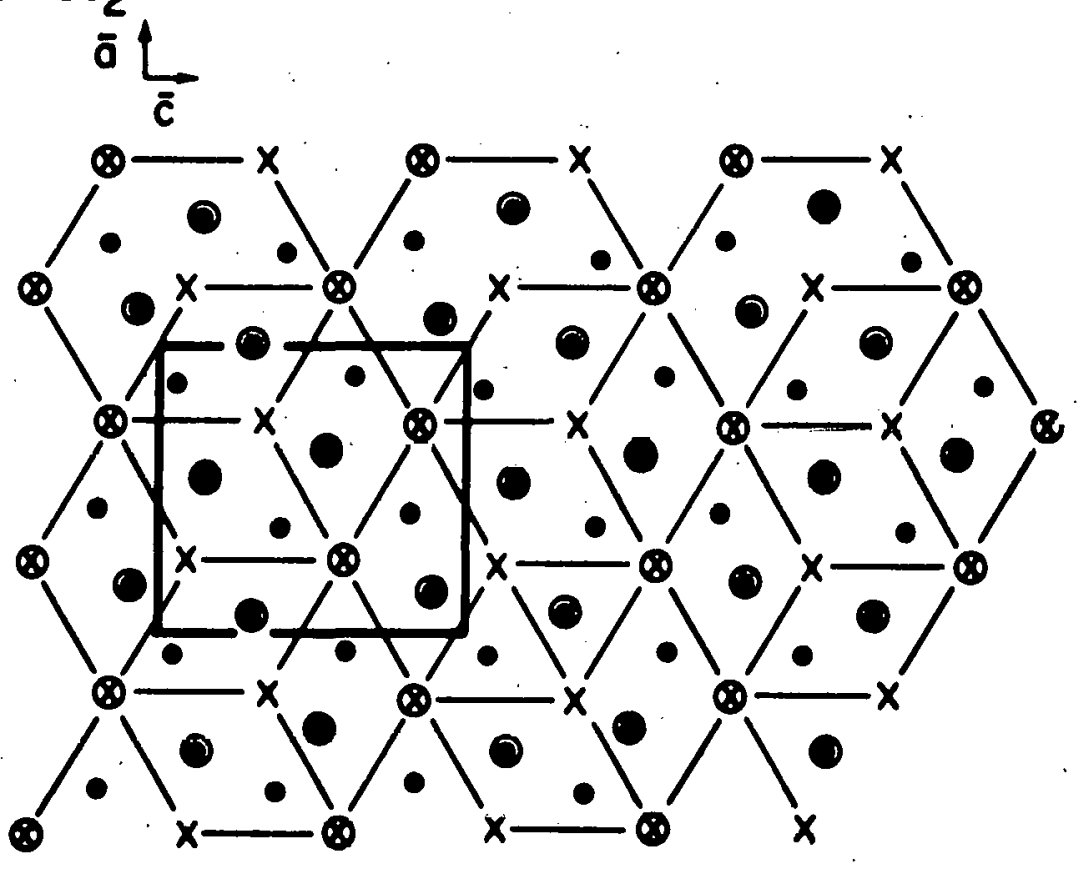

Figure 15. The stacking of the hexagonal rings in the $\mathrm{Fe}_{2} \mathrm{P}(\mathrm{a})$ and $\mathrm{CO}_{2} \mathrm{P}(\mathrm{b})$ structure types.

Orientations for the approximate unit cells are given by the dark lines 
VII. QUALITATIVE BONDING CONSIDERATIONS

\section{A. Review of Qualitative Bonding Considerations}

Conard (1) and Smeggil (2) reviewed the early ideas of Slater, Pauling, and Rundle concerning the nature of chemical bonding in solid materials. Slater (54) suggested that it is possible, even in the case of $\mathrm{KCl}$, to interpret the bonding of solids in terms of covalent interactions. The interpretation of solids in terms of covalent bonding is basic to the understanding of the bonding in this class of compounds.

For example, the concept of the delocalization of electrons in the chemical bonds of the solid is essential to the understanding of the physical properties of solids. Pauling introduced the resonance concept as an interpretation of the delocalization of bonding electrons in metals and alloys (53). The directional nature of the chemical bonds, implicit in the structures of the metals, suggested to Pauling the use of a hybrid orbital bonding scheme to account for the observed structures and properties. Rundle applied many of the same basic concepts to formulate a bonding scheme that would account for the observed properties of the transition metal nitrides and oxides (55). He assoclated the electrical conductivity of these phases with the delocalization of the bonding electrons and associated the brittleness of these phases with the directional nature of the bonds, 
describing the bonding orbitals in terms of a hybrid orbital model.

Franzen (51) applied the same ideas to the transition metal monochalcogenides, accounting for the observed metallic conductivity and brittleness in terms of a delocalized, directional, covalent bonding description. In order to account for the high coordination number observed for the nonmetal in these phases, Franzen proposed that the sulfur d-orbitals contribute significantly in the formation of the nonmetal-metal bonds. With these ideas as a guide, Conard and Smeggil discussed the predominant structural features of the metal-rich chalcogenide and pnictide phases known at the time.

In the bonding descriptions of Rundle, Pauling, and Franzen, there is a critical dependence of the bonding interpretation on the physical properties of the solids considered. The properties of this layered class of compounds outlined above suggest that the same basic concepts can be applied in the attempt to interpret the chemical bonding of the solids in this class. The following discussion can be viewed as an extension of these bonding ideas in an attempt to discover the role that the metal atoms might play in the chemieal bonding. The appruach is based upon the assumption that it is the nature of the metal partlal coordination polyhedra which offers an insight into 
the contribution of the metal atoms to the metal-nonmetal bonds.

The early work of Engel (56) and the later development by Brewer (57) of Engel's observations led to the correlation of metal structure with the number of $\underline{s}$ and $\underline{p}$ electrons avallable for bonding in metals and alloys. If $\mathrm{n}$ represents the number of $\underline{s}+\underline{p}$ electrons available in the metal, the correlation can be expressed by the following inequalities:

$$
\begin{array}{ll}
1.0 \leq n \leq 1.5 & \text { bcc metal structure } \\
1.7 \leq n \leq 2.1 & \text { hcp metal structure } \\
2.5 \leq n \leq 3.0 & \text { fcc metal structure }
\end{array}
$$

According to Hume-Rothery, Brewer has had a remarkable degree of success in using this correlation to predict the structures and phase diagrams for alloys of the transition metals (58):

In the qualitative bonding description that follows, there is no direct dependence on the Brewer-Engel Correlation as expressed above, but the bonding description rests on two basic assumptions of the Brewer-Engle Correlation as discussed by Brewer. First, that the structure of a transition metal compound is correlated with the electronic configuration of the ground state and low lying exclted states for the gaseous metal atom. The second assumption of the Brewer treatment concerns the slgnificance of the promotion energy of a gaseous metal atom from its ground 
state to an exc1ted state. When the ground state does not correlate with the observed structure for the metal while the excited state provides a correlation with the observed structure, a promotion is considered possible if the electronic configuration of the excited state can compensate for the required promotion energy by providing an increase in the bond energy. That is, the promotion energy is thought to be compensated by the increase in bond energy provided by an increase in the number of bonding electrons available in the excited state.

\section{B. Altmann, Coulson, and Hume-Rothery}

Altmann, Coulson, and Hume-Rothery (59) attempted to correlate the structures of the transition metals with the welght of d-orbital character available in valence orbitals centered on the metal atom. Since the different observed transition metal structures ( $f c c, b c c$, and hcp) correspond to different arrangements of fịst and second near neighbors abou't a central metal atom, Altmann, et al. "proposed a particular combination of hybrid orbitals for each of the structure types.. Each of these combinations of hybrid orbitals was characterlzed by a different weight of a-orbital contribution. By using the different welghts of d-orbital contribution corresponding to the different transition metal structure types, they were able to correlate the occurrence 
of the metal structures with the known behavior of the d-electrons in each of the three transition series.

There are several aspects of the Altmann et al. approach to the chemical bonding in the transition metals that will have direct application in the qualitative bonding considerations for the structures of the layered class. A detalled description of the important aspects of the Altmann et al. approach will be the subject of this section, and the following section will consist of the specific application of these ideas to the contribution of the transition metal orbitals to the Me-X bonds in the layered class of compounds.

In the valence bond approach of Pauling, Rundle, Brewer, and Altmann et al., the bonding contribution of particular metal atoms in a solid structure is considered to be correlated with the geometrical arrangement of neighboring atoms about the central atom. The recurrence of certain arrangements of atoms about a central atom (the P.C.P.) suggests that there is efficient bonding possible in the region of space between a central atom and the polyhedral configuration of atoms. Since hybrid orbitals centered on the central atom of a polyhedron can be chosen that provide a corresponding. Increase In the electron density in the direction of the neighboring atoms, the bonding contribution from a central atom will be approximated in terms of these ho's. Although the conduction electrons in the solld are 
known to be delocalized over the entire crystal, a distinction can be made between the conduction electron behavior and the behavior of the electrons in the region of space near the central atom of a polyhedron (e.g., in the muffin-tin sphere in the APW approach), and in this region the electrons will be referred to as bonding electrons. The symbol $\psi_{\text {bond }}$ will be introduced as an approximate wave function of the electrons in this region of space, where in $\psi_{\text {bond }} \propto \sum_{i} n_{i}\left(\varphi_{i}\right)$ and the $\varphi_{1}$ terms will represent the various ho's directed towards the atoms of the P.C.P. The $n_{i}$ coefficients will represent the relative contribution of each ho in $\psi_{\text {bond. The }}$ particular considerations used to choose the appropriate $\varphi_{i}$ terms will be considered as $\psi_{\text {bond }}$ expressions are introduced for the different partial coordination polyhedra.

\section{Symmetry considerations for b.c.c. metals}

In the b.c.c. metal structure a central metal atom has a cubic arrangement of eight first nearest neighbors and an octahedral arrangement of six second nearest neighbors. The particular ho's that will be used to approximate the bonding contribution from a central metal atom to the fourteen neighboring atoms are those suggested by Altmann et al. (59). Their approach will be considered in detail, as it is critical for understanding the symmetry considerations applied in limiting the ho's that may contribute to $\psi_{\text {bond }}$ for the P.C.P. of the layered class of compounds. 
In order to account for the bond formation to the cubic arrangement of eight nearest neighbors, Altmann et al. argue that either the $\underline{s d}^{3}$ or $\underline{d}^{4}$ ho's can be used. The gerade nature of the ho's (and thus the combined ho's) is used to provide eight bonding directions with cublc symmetry, where each direction corresponds to the eight lobes of the four $\underline{d}^{4}$ or $\underline{d}^{3} \underline{s}$ ho's. That is, the electrons occupying the $\underline{d}^{4}$ or $\underline{d}^{3} \underline{s}$ ho's are considered to be shared with eight atoms forming at most 1/2-order bonds.

Simple group theoretical considerations can be applied to the $\underline{s d}^{3}$ ho's to see that they are consistent with the point symmetry of the metal atoms in the b.c.c. metal. In the $\mathrm{T}_{\mathrm{d}}$ point group either the $\underline{s p}^{3}$ or $\underline{s d}^{3}$ ( $\underline{s}_{\mathrm{x} y} \mathrm{~d}_{\mathrm{xz}} \mathrm{d}_{\mathrm{yz}}$ ) ho combinations can be used to describe a tetrahedral bonding arrangement, but only the $\underline{s d}^{3}$ ho combination is capable of forming eight equivalent $1 / 2$-bonds in a cubic arrangement. The distinction between the ho's depends on their different behavior under the inversion operation of the $o_{h}$ point group. In $O_{h}$ the s-orbital belongs to the totally symmetric representation, A $A_{1 g}$, the three degenerate p-orbitals form a basis set for the $\mathrm{T}_{1 \mathrm{l}}$ Irreducible representation, and the $d_{x y} d_{x z} d_{y z}$ orbitals form a basis set for the $T_{2 g}$ irrep. Since the $\underline{s}-$ and three d-orbitals are symmetric with respect to the inversion operation of $\mathrm{O}_{h}$, the $\underline{s d}^{3}$ ho combination can provide elght equivalent bonding directions with full 
cub1c symmetry. The three p-orbitals, on the other hand, are antisymmetric with respect to the inversion operation in $O_{h}$, indicating that the $\mathrm{sp}^{3}$ ho's do not have the proper symmetry to form eight cubic bonds.

Altmann et al. used similar arguments to show that the $d^{4}\left(d_{z} 2, d_{x y}, d_{x z}, d_{y z}\right)$ ho's can also form eight $1 / 2$-bonds of cubic symmetry and the $\underline{a}^{3}$ ho's can form six 1/2-bonds of octahedral symmetry.

\section{General symmetry considerations}

The considerations for the $\underline{\mathrm{sd}}^{3}$ ho's suggest that this treatment may be generalized for other hybrid orbitals with different symmetry properties. It appears that if a general hybrid combination, $s^{k} p^{l} d^{m}$, forms a reducible representation, $\Gamma_{\text {red }}$, in symmetry group $G$, then the $s^{k} p^{l} d^{m}$ ho's are appropriate for two ligand per orbital bonding with the symmetry of the semidirect product group $G \Lambda C_{i}$ or $G \Lambda C_{S}$ when the ho's are either symmetric or antisymmetric with respect to $C_{i}$ or $\mathrm{C}_{S}, \underline{1} . \underline{e}$, when all of the basis rurictions are symmetric or antisymmetric under the operation $i$ or $\sigma$. Treatment of the $\underline{s d}^{3}$ ho's above considered the particular case where $G \Lambda C_{i}$ corresponded to the $O_{h}$ point group. These symmetry considerations can be applied to the Me-Me bond arrangement of P.C.P. II to illustrate the treatment when the product group is of the form $G \Lambda C_{S}$. The six metal neighbors of the central atom in unit II form a 
trigonal prism with point group $\mathrm{D}_{3 \mathrm{~h}}$. (For the considerations here, the deviations from ideal $D_{3 h}$ symmetry 1mplied by the differences in bond length are 1gnored.) The point group $\mathrm{D}_{3 \mathrm{~h}}$ is the semidirect product group $\mathrm{C}_{3 \mathrm{v}} \Lambda \mathrm{C}_{\mathrm{s}}$ and the $\mathrm{C}_{3 \mathrm{v}}$ point group describes the symmetry for the trigonal pyramidal arrangement of bonds for one-half of a trigonal prism. The arrangement of the remaining three bonds is related to this trigonal pyramid by the mirror plane perpendicular to the $\mathrm{C}_{3}$-axis of the trigonal prism. The basis functions in $\mathrm{C}_{3 \mathrm{v}}$ corresponding to the reducible representation, $\Gamma_{\text {red }}=A_{I}+E$, are listed in the first two columns of Table 15. The signs of the characters of the two basis sets of atomic orbitals for the $\sigma_{h}$ operation of $D_{3 h}$ are indicated in the next two columns. A ho combination having the same sign in both columns has the proper symmetry to form six 1/2-bonds in the form of a trigonal prism. This can be conceptualized for the bonding in the trigonal prism by picturing the three positive lobes of a hybrid combination such as $d^{2} p\left(d_{x y} d_{y z} p_{z}\right)$ forming bonds to three atoms of the prism, while the corresponding negative lobes form bonds to the remaining three atoms of the prism. Although the $\underline{s p}^{2}$ and $\underline{s d}^{2}$ ho's in Table 15 obey the symmetry conditions, they probably do not provide enough of a bonding contribution in the z-direction to account for bonding in the trigonal prism. 
Table 15. Symmetry relationship between the $\mathrm{C}_{3 \mathrm{v}}$ and $\mathrm{D}_{3 \mathrm{~h}}$
point groups

\begin{tabular}{|c|c|c|c|c|}
\hline $\begin{array}{l}\text { Hybrid } \\
\text { Orbital }\end{array}$ & $\begin{array}{c}E+A_{1} \\
\text { Basis Sets } \\
\left(C_{3 . v}\right)\end{array}$ & $\begin{array}{l}\text { Sign of } E \\
\text { irrep. for } \\
\sigma_{h}\end{array}$ & $\begin{array}{c}\text { Sign of } A_{1} \\
\text { irrep. for } \\
\sigma_{h}\end{array}$ & $\begin{array}{l}\text { Same } \\
\text { Sign }\end{array}$ \\
\hline$p^{3}$ & $\left(p_{x}, p_{y}\right)+p_{z}$ & + & - & No \\
\hline$d^{2} p$ & $\left(d_{x}{ }^{2}-y, d_{x y}\right)+p_{z}$ & + & - & No \\
\hline$d^{2} p$ & $\left(d_{x y}, d_{y z}\right)+p_{z}$ & - & - & Yes \\
\hline$d p^{3}$ & $\left(p_{x}, p_{y}\right)+d_{z}^{2}$ & + & + & Yes \\
\hline$d^{3}$ & $\left(d_{x}{ }^{2}-y^{2}, d_{x y}\right)+d_{z^{2}}$ & + & + & Yes \\
\hline$d^{3}$ & $\left(d_{x z}, d_{y z}\right)+d_{z} 2$ & - & + & No \\
\hline$s p^{2}$ & $\left(p_{x}, p_{y}\right)+s$ & + & + & Yes \\
\hline$s d^{2}$ & $\left(d_{x^{2}-y^{2}, d_{x y}}\right)+s$ & + & + & Yés \\
\hline$s d^{2}$ & $\left(d_{x z}, d_{y z}\right)+s$ & - & + & No \\
\hline
\end{tabular}

3. $\psi_{\text {bond }}$ for the b.c.c. metal

As an approximation for the bonding contribution of a metal atom in the b.c.c. metal, $\psi_{\text {bond }}($ bcc $)$ will be written as $\psi_{\text {bond }}(b c c) \propto n_{1}\left(s d^{3}\right)_{1}+n_{2}\left(d^{4}\right)_{1}+n_{3}\left(d^{3}\right)_{1}+\cdots$ since symmetry considerations cannot distinguish whether the sd $^{3}$ or $\underline{d}^{4}$ ho's might make the larger contribution to $\psi_{\text {bond }}(b c c)$, 
both combinations will be retained. In this expression for $\psi_{\text {bond }}$, and In all similar expressions that will be considered, the presence of a subscript $i$ or $m$ with $a$ contributing ho signifies two ligand per orbital bonding for the hybrid orbital. The specific use of the subscript $i$ identifies precisely which lobes of a ho are considered to be used in bond formation, the 1 signifying that the bonding lobes are related by an inversion operation. A subscript $m$ will identify those hybrid combinations such as $\underline{d}^{2} \underline{p}$, where the bonding lobes are related by a mirror plane. If no subscript is indicated for a ho in $\psi_{\text {bond }}$, the hybrid combination is considered to form one ligand per orbital bond.

The coefficients in $\psi_{\text {bond }}(b c c)$ above represent the relative contribution of each of the ho's. Although the number of electrons in a particular ho is unknown, the number contributing to the bonds represented by a ho will be considered to be related to the coefficient $n_{i}$ in a general way. If $\psi_{\text {bond }}$ could accurately be written with only one term, such as $n_{i}\left(s^{3}\right)_{1}$, the value of $n_{1}$ would be 1.0 and the four or less electrons implicit in writing $\underline{s d}^{3}$ would all be considered to be involved in bond formation to the eight cubic atoms. However, $\psi_{\text {bond }}$ will always contain a number of terms, and the division of electrons among the various terms is unknown. 
For example, if $\psi_{\text {bond }}$ could be written with only $\mathrm{n}_{1}\left(\mathrm{sd}^{3}\right)_{1}+\mathrm{n}_{2}\left(\mathrm{~d}^{3}\right)_{1}$ making a bonding contribution, symmetry considerations do not specify whether $n_{1}$ or $n_{2}$ is larger. However, in the b.c.c. metals the difference in interatomic distances to first and second near nelghbors suggest that $n_{1}$ is larger than $n_{2}$. In Section $D$ below, simple energy considerations will be presented which suggest additional limitations for the $n_{i}$.

Altmann et al. (59) emphasized that although the particular choice of ho's in $\psi_{\text {bond }}$ depends primarily on the geometrical arrangement of neighbors about a central atom, the choice of $\varphi_{1}$ does not mean that there are no other terms that may contribute to the description of the electrons in the region near the central atom. The other terms that contribute to the wave function are, however, assumed to have no effect on the geometrical arrangement of neighboring atoms. Brewer (57) considers this same point and emphasizes that the contribution from other terms can be substantial. Since these additional terms will not be stated explicitly, their presence will be indicated by writing $\psi_{\text {bond }}$ as an open sum.

In writing $\psi_{\text {bond }}$ expressions for the P.C.P., the large number of possible terms with their corresponding uncertainty in the value of $n_{1}$ suggests that the coefficients may be relatively small. If $\psi_{\text {bond }} \propto n_{1}\left(d^{2}\right)_{1}+n_{2}(d)+\cdots$ were 
written to account for bonding to part of a P.C.P., the expression should in no way be construed to indicate a valence on the order of two. Rather, it must be kept in mind that this approach emphasizes the directional nature of the bonding contribution of a central atom.

$$
\text { C. Application to the Metal P.C.P. }
$$

The application of the bonding considerations presented in the last section to the metal P.C.P. of the layered class involves an extension of the symmetry arguments presented by Altmann et al. In any consideration of $\psi_{\text {bond, a choice has }}$ to be made concerning which of the ho's might be expected to make a substantial contribution. Such a choice must necessarily be based on energy considerations. For the structures of this class information about the bond energies, enthalpy of formation, entropy, etc. are not available, so only indirect consideration can be given to the complex energetic factors that may be involved in determining phase stability. The structural features of this class of compounds, when combined with simple chemical. considerations, indicate that certain of the ho's may be expected to make a greater contribution in $\psi_{\text {bond }}$ than others. There remains, of course, a degree of arbitrariness in any choice of the $\varphi_{i}$ in $\psi_{\text {bond }}$. 
1. Application to P.C.P. II

In applying the bonding considerations of Altmann et al. to a particular metal partial coordination polyhedron, the possible hybrid orbitals that are consistent with the arrangement of atoms about the central atom are listed. Even if the various P.C.P. are approximated by geometrically regular units, there are still a relatively large number of ho's that are consistent with the point symmetry for the central atom of a particular P.C.P. For example, $\psi_{\text {bond }}$ for unit II in equation 17 contains a large number of ho combinations which are all consistent with the symmetry considerations.

$$
\begin{aligned}
\psi_{\text {bond }}(I I) \propto & n_{1}\left(d^{3}\right)+n_{2}\left(d^{3} p\right)+n_{3}\left(d^{2} p^{2}\right)+n_{4}\left(d^{4}\right) & \\
& \quad+n_{5}\left(d s p^{2}\right)+n_{6}\left(d^{3} s\right) & \text { Me-X } \\
& +n_{7}\left(d^{2} s p^{3}\right)+n_{8}\left(d^{4} s p\right)+n_{9}\left(d^{3} p^{3}\right)+n_{10}\left(d^{5} p\right) & \text { Me-Me } \\
& +n_{11}\left(d^{2} p\right)_{m}+n_{12}\left(d p^{2}\right)_{m}+n_{13}\left(d^{3}\right)_{m} & \text { Me-Me } \\
& +n_{14}\left(d^{2}\right)_{1}+n_{15}(s d)_{1}+\cdots & \text { Me-X }
\end{aligned}
$$

There are several points to be made concerning this expression for $\psi_{\text {bond }}(I I)$. The various $\Psi_{1}$ are listed in four separate lines which are distingulshed by a oymbol to the right of each line which corresponds tin the naturc of the bonding interaction. The first line of equation 17 simply indicates the ho combinations which could account for bond formation to the four nonmetal nelghbors in P.C.P. II where 
their arrangement is approximated by a square pyramid with $\mathrm{C}_{4 \mathrm{v}}$ symmetry. The second line lists the four ho combinations consistent with the arrangement of six metal neighbors in the form of a trigonal prism, $\mathrm{D}_{3 h}$. The ho's in line three can also be used to account for the trigonal prismatic bond arrangement to metal neighbors, and these terms correspond to those listed in Table 17 and discussed above. The last line represents a possible choice of ho combinations that could account for bond formation to the four nonmetal neighbors in unit II by utilizing the gerade nature of the specified ho combination to form two ligand per orbital bonds.

2. Simple energy considerations to limit $\psi_{\text {bond }}$ (II)

The Brewer-Engel correlation emphasizes the dependence of metal structure on the electronic configuration of the low lying electronic states of the various metals. The high degree of success of the Brewer-Engel correlation suggests that similar considerations might be applied to the metal P.C.P. In an attempt to determine which of the $\boldsymbol{\varphi}_{i}$ might be expected to make stronger contributions to $\psi_{\text {bond }}$. Any such consideration of the electronic states requires specification of the particular transition metal, since the electronlc configurations vary for different metal atoms. For titanium atoms the low lying electronic conflgurations can be represented as $s^{2} d^{2}$ (ground state), $s d^{3}$ ( $19 \mathrm{kcal} / \mathrm{mole}$ ), 
$d^{2} \mathrm{sp}\left(45 \mathrm{kcal} / \mathrm{mole}, \mathrm{d}^{3} \mathrm{p}(75 \mathrm{kcal} / \mathrm{mole})\right.$, etc., where the energy assoclated with the varlous conflgurations is the promotion energy for gaseous t1tanium atoms from the ground state to the lowest energy term for each configuration. The various terms and energies are obtalned. from Moore's Tables $(60)$.

In the approach used here, an assumption is made that the ho combinations that make substantial contributions to $\psi_{\text {bond }}$ must correspond to a low lying electronic configuration of the gaseous metal atom, and if more than one ho combination corresponds to the low lying electronic states, their relative contribution depends on the energy differences between the electronic states.

For example, the ho combinations in the first two lines of equation 17 correspond to electronic configurations whose promotion energy is unknown or expected to be higher than the energies for the low lying electronic states of titanium listed above. Of the six ho combinations listed in line one of equation 17 , all but the $d^{3}$ s term would be expected to have promotion energies greater than approximately $70 \mathrm{kcal} /$ mole. Since each of these six ho combinations contains the same number of electrons, none of them might in a first approximation be expected to provide substantially more bond energy than the others (6I). The consideration of promotion energy and approximate bond energies suggests that the $\mathrm{sd}^{3}$ ho 
combination might be expected to make a substantially larger contribution to $\psi_{\text {bond }}$ than the other terms in the first line of equation 17 .

Similar consideration for the terms in line three of equation 17 suggests that the bonding contribution from the $d p^{2}$, and perhaps the $d^{2} p$, ho combinations may not be as important as the contribution from the $\mathrm{d}^{3}$ combinations. In this case, the $\mathrm{d}^{3}$ ho is considered to make a larger bonding contribution than the $d^{2} p$ combination, since the $d^{2} p$ combination corresponds to the $d^{3} p$ electronic state with a $75 \mathrm{kcal} / \mathrm{mole}$ promotion energy, while the $\mathrm{d}^{3}$ ho combination corresponds to the $\mathrm{d}^{3} \mathrm{~s}$ excited state having a promotion energy of only $19 \mathrm{kcal} / \mathrm{mole}$.

Using these simple energy considerations to limit the contribution of the ho's in $\psi_{\text {bond }}$ (II) suggests that equation 17 may be rewritten to correspond to the particular case of titanium metal.

$$
\begin{aligned}
\psi_{\text {bond }}(I I) \propto n_{1}\left(d^{3} s\right) & +n_{2}\left(d^{2}\right)_{1}+n_{3}(s d)_{1} \\
& +n_{4}\left(d^{3}\right)_{m}+\cdots
\end{aligned}
$$

The procedure given here to limit the contributions to $\psi_{\text {bond }}(I I)$ for titanium metai can also be applied for the other transition metals. A difference in the low lying electronic states for the different metals would lead to an expression similar to equation 18 but differing in the particular $\Psi_{i}$ listed; the choice of $\varphi_{1}$ depending on the 
particular electronic configurations for each metal. This entire procedure can be applied to each of the different metal P.C.P., resulting in a set of expressions for $\psi_{\text {bond }}$ corresponding to each of the different P.C.P. where each member of a set represents a different transition metal. To list each individual $\psi_{\text {bond }}$ would require a prohibitive amount of space; so instead, consideration will be given to the structural features of the phases in this class which may suggest additional limitations on $\psi_{\text {bond }}$.

3. Implication of the occurrence of the same P.C.P. for different metals

In the earlier discussions of the metal P.C.P., the occurrence of the same P.C.P. for different metals in a variety of different phases with different stoichiometry suggested that there is a strong similarity in the nature of the bonding contribution of the different transition metals despite differences in their electronic configurations: Comparison of the different. "bond expreseions for a melal P.C.P. where different transition metals are involved indicates that the common terms in each expression are the ho combinations, such as $\left(d^{2}\right)_{1},(d s)_{1},(d),\left(d^{3}\right)_{1},\left(d^{3}\right)_{m}$, etc.

Occasionally, a $\varphi_{1}$, such as $\left(d^{3} \mathrm{~s}\right)$ in equation 17 which was proposed as an appropriate combination for Me-X bonding In unit II, occurs for the majority, but not all, of the, 
transition metals. For example, unit $V$, which has the same arrangement of Me-X bonds as unit II, is the predominant metal P.C.P. in the $\mathrm{Rh}_{4} \mathrm{P}_{3}$ phase. However, the low lying electronic states for rhodium metal $\left(d^{8} s, d^{9}, d^{7} s^{2}, d^{8} p\right.$, etc.) do not provide a $d^{3}$ s term which might have been proposed as yielding ho's appropriate to the Me-X bonds. Within the framework of this model, it is the use of terms such as $\left(d^{2}\right)_{i},\left(d_{3}\right)_{m}$, etc. that can account for the Me-X bond formation in $\mathrm{Rh}_{4} \mathrm{P}_{3}$. It is these ho combinations that are avallable for use in bond formation by all of the transition metal atoms in the sense that they correspond to a low lying electronic state for all of the metal atoms. The use of these ho combinations $\left(a_{i}^{2}, d_{m}^{3}, d\right.$, etc.) by the different metals provides one explanation of why the different metal atoms are observed to form the same P.C.P.

4. Occurrence of different.P.C.P. for the same metal

The occurrence of different types of partial coordination polyhedra for the salle inetal within the same phase indicates a flexibility of the metal in the formation of its bonds. In $\mathrm{Ti}_{2} \mathrm{~S}$, the metal has P.C.P. of types I, II, IV, VI, and VII, which differ markedly in both the type and geometrical arrangements of bonds to neighboring atoms. The differences in the metal bonding contribution for different P.C.P. can be thought of within the framework of this model 
as the utilization of different hybrid orbital combinations in bond formation.

As an example, consider the different $\psi_{\text {bond }}$ expressions. for units I and II for titanium metal

$$
\begin{aligned}
& \psi_{\text {bond }}(I) \propto n_{1}\left(s d^{3}\right)_{1}+n_{2}\left(d^{3}\right)_{1}+\cdots \\
& \psi_{\text {bond }}(I I) \propto m_{1}\left(d^{2}\right)_{1}+m_{2}\left(d^{2} p\right)_{m}+m_{3}\left(d^{3}\right)_{m}+\cdots
\end{aligned}
$$

The two different expressions represent different divisions of electron concentration among different ho combinations. In $\psi_{\text {bond }}(I)$, the majority of the bonding electron concentration is considered to occupy the $\mathrm{sd}^{3}$ ho's, while for $\psi_{\text {bond }}(I I)$ the electron contribution from the metal atom can be thought to be shared between the $\left(d^{2}\right)_{1}$ and $\left(d^{3}\right)_{m}$ terms. For P.C.P. II, the division of electrons does not have to be made equally between the two terms. (The differences in TI-TI and Ti-S bond orders suggests that $m_{1}$ is significantly larger than $\mathrm{m}_{2}$ even though both terms are considered to be important.)

The explanation of the flexibility of the metal in bond formation in terms of the use of different d-orbital comb1nations depends on the avallability of partially filled d-orbitals for a particular metal. The metals forming structures of the layered class all have in common partially filled d-orbitals. It is for the metals of the $\mathrm{N1}, \mathrm{Cu}$, and Zn families where the d-orbital orbital filling approaches 
completion, that the metals form structures of a different class. Within the framework of this model, the change in structure type for these families is thought to be dependent upon the nonavailability of d-orbitals for use in bond formation.

The basic question as to why one metal atom in a structure forms one P.C.P. while a second forms a different P.C.P. Is far from totally understood, but is expected to show a strong dependence on the nature of the Me-X interaction. Such an interaction depends in part on the individual contribution of each atom to the bond, but the interaction might also be expected to depend on some as yet undefined compatibility relationship between the two individual atom contributions. For example, it may be expected that the metal bonding contribution is itself dependent on the bonding contribution of the neighboring atoms. It is the nature of this interdependence which remains undefined.

It is hoped that any future study of the bonding in the structures of the layered class will center on defining the nature of this interaction. The importance of atom point symmetry discussed here suggests that the interdependence of any atom with its neighborlng atoms may be defined, or at least limited, by symmetry correlation rules applicable to the atom point groups involved. 


\section{Qualitative Bonding Considerations and Structural Features}

There is a strong interdependence between the qualitative bonding considerations and the structural features observed for the layered class of compounds. In this section, an attempt will be made to discuss certain of the structural features in terms of the qualitative model presented above. This approach will suggest some additional limitations on $\psi_{\text {bond }}$ and will provide a possible explanation of certain other structural features.

1. $\Psi_{\mathrm{Me}-\mathrm{X}}$ and the octahedral P.C.P.

In dividing the metal P.C.P. in terms of their number and orientation of $\mathrm{Me}-\mathrm{X}$ bonds, it was suggested that the qualitative bonding description should account for the similarity among the $\mathrm{Me}-\mathrm{X}$ bond arrangements in the various P.C.P. and the octahedral Me-X bond arrangements in unit VIII. Such a comparison, however, is dependent upon the bonding scheme used to describe unit VIII.

The distorted octahedral bond arrangement in unit VIII might be described in terms of $\mathrm{d}^{2} \mathrm{sp}^{3}$ ho combinations (or alternately in terms of $\mathrm{d}^{3} \mathrm{p}^{3}$ ho combinations if the bond arrangement 1s approximated as a trigonal ant1-prism). Simple energy arguments were used above to suggest that the use of the $d^{2} \mathrm{sp}^{3}$ or $\mathrm{d}^{3} \mathrm{p}^{3}$ ho combinations might not be the 
best way to account for the distorted octahedral arrangement of bonds in unit VIII. By arguing that the promotion energy for a metal such as niobium or vanadium to a $d^{2} s p^{3}$ state was very large, an assumption was made that there would be six electrons involved in bond formation and that the sixth electron would require promotion from a filled subshell.

This assumption is certainly not necessarily valid. Arguments can be made that there may be less than six electrons occupying the six $d^{2} \mathrm{sp}^{3}$ ho's with a large decrease in the required promotion energy, since the partial occupancy of the ho combinations might no longer require promotion of an electron from a filled subshell. If the number of bonding electrons is considered to decrease in this way, then there will also be a corresponding decrease in the bond energy, but there will certainly be cases where the decrease in promotion energy exceeds the decrease in bond energy and a specific configuration will become more important in bond formation than first expected.

As a specific example, an approximation can be made for the promotion energy required for nioblum metal to undergo a hypothetical transformation from $\mathrm{sd}^{4}$ to $\left(\mathrm{d}^{2} \mathrm{sp}\right)^{3 / 6}$, where the electronic state represented by $\left(d^{2} s p^{3}\right)^{5 / 6}$ corresponds to five electrons occupying the six $d^{2} s p^{3}$ ho's. This particular transformation would require promotion of approximatly $2-1 / 2$ electrons from a $\underline{d}$ to a $\underline{p}$ orbital. The energy required for 
the promotion of one electron from a $\mathrm{d}$ to a $\underline{p}$ orbital might be estimated as $44 \mathrm{kcal} / \mathrm{mole}$ where this value corresponds to the promotion energy for the transformation of gaseous niobium atoms from the $d^{4} s$ ground state to the $d^{3} s p$ excited state. Based on these considerations, a reasonable estimation of the promotion energy required for the transformation $s d^{4} \rightarrow\left(d^{2} \mathrm{sp}^{3}\right)^{5 / 6}$ would be on the order of $100 \mathrm{kcal} / \mathrm{mole}$. In considering the bond energies for the various transition metals, Brewer $(57,61)$ and Altmann et al. (59) both argue that the bond energy depends much more on the number of bonding electrons than their orbital character. The $d^{4} s$ configuration with five electrons and the $\left(d^{2} s p^{3}\right)^{5 / 6}$ configuration, also with five electrons, can be expected to have approximately the same bond energy but with the latter favored because of the more effective overlap of $p$ relative to d orbitals $(57,62)$. For nioblum the bonding contribution from the $d^{4} s$ configuration may be expected to be more important than the contribution from $\left(d^{2} \mathrm{sp}^{3}\right)^{5 / 6}$ because of the difference in promotion energy, and because the $d^{4} s$ configuration can also account for the octahedral arrangement of nonmetal atoms in unit VIII by using the $\mathrm{d}^{3}$ or $\mathrm{d}^{2} \mathrm{~s}$ ho's to form two ligand per orbital bonds.

Equation $20 a$ describes the metal bonding contribution utilizing the $\mathrm{d}^{3}$ ho combination and two ligand per orbital bonding. Equations $20 \mathrm{a}$ through $20 \mathrm{e}$ illustrate the similarity 
in the Me-X bond arrangements for units II through VII with each other as well as with the distorted octahedral bond arrangement in unit VIII.

$$
\begin{aligned}
& \psi_{\text {Me-X }}(\text { VIII }) \propto n_{1}\left(d^{3}\right)_{1}+\cdots \\
& \psi_{\text {Me-X }}(\text { VI }) \propto n_{1}\left(d^{2}\right)_{i}+n_{2}(d)+\cdots \\
& \psi_{\text {Me-X }}(I I, V) \propto n_{1}\left(d^{2}\right)_{i}+\cdots \\
& \psi_{\text {Me-X }}(\text { VII }) \propto n_{1}\left(d^{2}\right)_{i}+n_{2}(d)+\cdots \\
& \psi_{\text {Me-X }}(I I I, \text { IV }) \propto n_{1}(d)_{m}+\cdots
\end{aligned}
$$

In equation 20a, the distorted octahedral arrangement of Me-X bonds in unit VIII can be described in terms of bond formation by six lobes of the $d^{3}$ ho's. For unit VI only five of the six lobes are used to form Me-X bonds, and the nature of the bonding contribution differs for the two terms in equation $20 \mathrm{~b}$. The expression in equation $20 \mathrm{c}$ indicates that for units II and $V$ only four of the six lobes of the $\mathrm{d}^{3}$ ho's are utilized in formation of Me-X bonds. For unit VII the description becomes more complicated and the arbitrary division of $\psi_{\text {bond }}$ into Me-Me and Me-X components less accurate. The description of unit VII in terms of unit VI above indicated that the only difference was replacement of two nonmetal atoms in unit VI by metal atoms in unit VII. This relationship can be expressed by writing $\psi_{\text {bond }}$ (VII) exactiy the same as $\psi_{\mathrm{Me}-\mathrm{X}}(\mathrm{VI})$, equation $20 \mathrm{~b}$, if it is kept in mind that the $\left(d^{2}\right)_{i}$ term now accounts for bonding 
two metal and two nonmetal atoms instead of bonding to four nonmetal neighbors as in $\psi_{\text {bond }}(V I)$.

The description for the bonding electrons of the central atom in units VI and VII 1llustrates that the electrons associated with the $n_{1}\left(d^{2}\right)_{1}$ term are delocalized over four nonmetal atoms, while the electrons corresponding to the $\mathrm{n}_{2}$ (d) term are not delocalized in two ligand per orbital bonds. Thus, the electron concentration associated with the $\mathrm{n}_{2}$ (d) term can be greater than for $\left(d^{3}\right)_{1},\left(d^{2}\right)_{1}$, etc., since there is no longer a limitation to formation of two $1 / 2$ order bonds. This approach provides one possible explanation for the observed difference in the Me- $\mathrm{X}$ bond lengths in units VI and VII.

\section{Differences in interatomic distances}

In any covalent bonding model such as the one considered here, an interatomic distance is expected to be related in. some way to the electron concentration contributed to the bond from buth of the atoms that form that chemical bond. In the layered class of compounds, the interatomic distances for each of the metal P.C.P. can be expected to depend on the contribution of electrons from the central atom as well as the contribution from each of the nelghboring atoms to the bonds. Earlier it was illustrated that for each of the different P.C.P., both the interatomic distances from a central atom to the polyhedral atoms and the type of P.C.P. 
of the polyhedral atoms vary in the structures of the layered class. Figure 16 illustrates the difference in the types of P.C.P. for those atoms which are polyhedral atoms for unit I In the $\mathrm{Ti}_{2} \mathrm{~S}$ and $\mathrm{TI}_{8} \mathrm{~S}_{3}$ structures. A question can be raised concerning a possible correlation between the difference in interatomic distances observed for unit $I$ and the type of P.C.P. observed for the polyhedral atoms of unit I.

Since the number of polyhedral atoms varies for different types of metal P.C.P., it might be expected that the average number of electrons contributed to bonds by the central atoms depends on the number of neighbors. For example, the Me-Me bond lengths in unit I with eight neighbor atoms and unit II with ten neighbors might vary because of a difference in the average contribution of electrons from a central atom to the bonds. $\psi_{\text {bond }}(I)$ for titanium, written as $n_{1}\left(s d^{3}\right)_{1}+n_{2}\left(d^{3}\right)_{1}+\cdots$, suggests that the contribution of electrons from the central atom to its neighbors might, as a first approximation, be considered to be shared equally among the elght polyhedral atoms. If this assumption is valid, and the contribution of electrons from the eight polyhedral atoms to the central atom of unit $I$ does depend on the number of atorns in the P.C.P. of the polyhedral atoms, the interatomic distances In unit I should show variation with the type of P.C.P. found for the polyhedral atoms. 
The six titanium atoms in the P.C.P. of type I in $\mathrm{TI}_{2} \mathrm{~S}$ and $\mathrm{Ti}_{8} \mathrm{~S}_{3}$ exhibit six different types of Partial Coordination Polyhedra as illustrated in Figure 16. The different types of P.C.P. contain different numbers of near neighbors; eight for P.C.P. I and IV, nine or ten for P.C.P. II, V, VI and VII. Interatomic distances from the central atom of unit I to the polyhedra atoms are divided in Table 16 based on the number of near neighbors for each polyhedral atom. Units I and IV with only elght atoms in their P.C.P. exhibit substantially shorter Me-Me interatomic distances than observed for the other units with nine or ten atoms in their. P.C.P. Thus, the inclusion of the bonding contribution from polyhedral atoms as well as the central atom provide one possible explanation of the variance in interatomic distances for unit $\mathrm{I}$ in $\mathrm{TI}_{2} \mathrm{~S}$ and $\mathrm{TI}_{8} \mathrm{~S}_{3}$ and suggest application to other Me-X systems.

Since the qualitative bonding considerations presented here do not quantitatively account for differences in interatomic distances, the question might be asked, "Do the observed differences in interatomic distances for a P.C.P. suggest further limits on the $\varphi_{1}$ and $n_{1}$ in $\psi_{\text {bond }}$ ?" The answer to this question has not been considered to the degree to which it might. The difficulty in answering this question is related to the tremendous amount of data for interatomic distances that would have to be tabulated and 
Figure 16. An iliustration of the differences in the types of P.C.P. (broken lines) for those atoms (solid circles) which are the polyhedral atoms for P.C.P. I (solid lines) in the $\mathrm{Ti}_{2} \mathrm{~S}$ and $\mathrm{TI}_{8} \mathrm{~S}_{3}$ structure types 

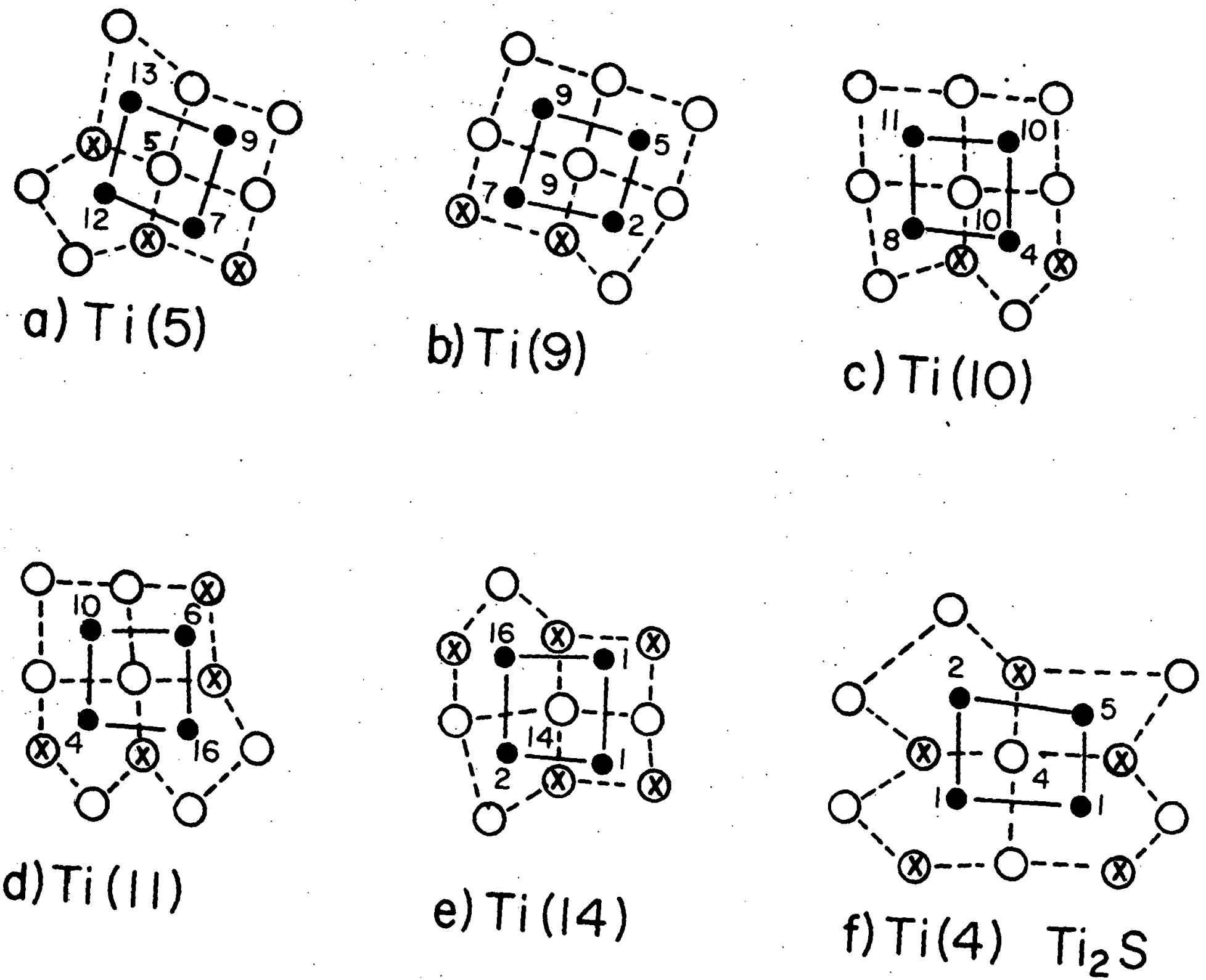
Table 16. Difference in interatomic distances for atoms with a different number of near neighbors (Reference Figure 16)

\begin{tabular}{|c|c|c|c|c|c|c|c|c|}
\hline \multirow[b]{2}{*}{ Phase } & \multirow{2}{*}{$\begin{array}{l}\text { Central } \\
\text { Atom }\end{array}$} & \multicolumn{3}{|c|}{$\begin{array}{l}\text { Polyhedral Atoms with } \\
\text { Eight Near Neighbors }\end{array}$} & \multicolumn{4}{|c|}{$\begin{array}{l}\text { Polyhedral Atoms with } \\
\text { Nine or Ten Near Neighbors }\end{array}$} \\
\hline & & Atom & Interatomic & Distance. & Atom & Interatomic & Distan & hee \\
\hline $\mathrm{T}_{8} \mathrm{~S}_{3}$ & $\mathrm{~T} 1(5)$ & $\operatorname{Ti}(9)$ & 2.770 & & $\begin{array}{l}\operatorname{Ti}(7) \\
\operatorname{T} 1(12) \\
\operatorname{Ti}(13)\end{array}$ & $\begin{array}{l}2.905 \\
2.930 \\
2.922\end{array}$ & & \\
\hline $\mathrm{Ti}_{8} \mathrm{~S}_{3}$ & $\mathrm{~T} 1(9)$ & $\begin{array}{l}\operatorname{Ti}(5) \\
\operatorname{Ti}(9) \\
\operatorname{Ti}(2)\end{array}$ & $\begin{array}{l}2.770 \\
2.696 \\
2.803\end{array}$ & & $\mathrm{~T} 1(7)$ & 2.955 & & \\
\hline $\mathrm{Ti}_{8} \mathrm{~S}_{3}$ & $\operatorname{TI}(10)$ & $\begin{array}{l}\mathrm{Ti}(10) \\
\mathrm{Ti}(11) \\
\mathrm{Ti}(8)\end{array}$ & $\begin{array}{l}2.710 \\
2.843 \\
2.767\end{array}$ & & $\mathrm{Ti}(4)$ & 2.943 & & \\
\hline $\mathrm{Ti}_{8} \mathrm{~S}_{3}$ & $\mathrm{~T} 1(11)$ & $\mathrm{T} 1(10)$ & 2.843 & : & $\begin{array}{l}\operatorname{Ti}(6) \\
\operatorname{Ti}(16) \\
\operatorname{Ti}(4)\end{array}$ & $\begin{array}{l}2.903 \\
2.995 \\
2.938\end{array}$ & & \\
\hline $\mathrm{Ti}_{8} \mathrm{~s}_{3}$ & $T 1(14 i$ & $\mathrm{Ti}(2)$ & 2.783 & 3 & $\begin{array}{l}\operatorname{Ti}(16) \\
\operatorname{Ti}(1) \\
\operatorname{Ti}(1)\end{array}$ & $\begin{array}{l}2.942 \\
2.901 \\
2.820\end{array}$ & 2 & \\
\hline \multirow[t]{2}{*}{$\mathrm{Ti}{ }_{2} \mathrm{~S}$} & $\mathrm{Ti}(4)$ & & & & $\begin{array}{l}\mathrm{T} 1(1) \\
\mathrm{T} i(1) \\
\mathrm{T} i(2) \\
\mathrm{T} 1(5)\end{array}$ & $\begin{array}{l}2.843 \\
2.952 \\
2.953 \\
2.838\end{array}$ & & . \\
\hline & & Mear: & 2.776 & . & Mean: & 2.916 & & \\
\hline
\end{tabular}


compared in the proper way. Another difficulty is that this approach may place too much emphasis on the importance of relatively small differences in bond lengths. On the other hand, there are indications suggesting that the application of this approach may provide some interesting insights about the nature of the metal contribution to its chemical bonds. For several of the phases there are trends in interatomic distances that might be correlated with a particular choice of $\varphi_{i}$ in $\psi_{\text {bond }}$, but the trends in bond distance are not as striking as the difference in the Me-X bond distances for units VI and VII.

\section{E. Qualitative Model and Point Group Symmetry}

In the structures of the layered class, the mirror plane is the only symmetry element present in the point group for every atom position of each structure type. It was the presence of this symmetry element that suggested that the qualitative bonding considerations presented here should in some way ut1lize the mirror plane. The discussion of the Me-Me bonds in P.C.P. II utilizes the $\left(d^{2} p\right)_{m}$ term to account for the trigonal prismatic arrangement of metal atoms. Similar bonding contributions could also have been used to deccribe the bonding in other types of P.C.P. Th1s general approach of relating the bonding contribution of a central atom to 1ts point symmetry suggests application of these 
same ideas to structures which are not members of the layered class and which have atom positions with different point symmetry.

1. The $\mathrm{Ta}_{2} \mathrm{P}$ and $\mathrm{Hf}_{2} \mathrm{~S}$ s.tructure types

Both Conard (1) and Smeggil (2) consider the differences in the $\mathrm{Ti}_{2} \mathrm{~S}\left(\mathrm{Ta}_{2} \mathrm{P}\right.$ structure type) and $\mathrm{Hf}_{2} \mathrm{~S}$ structure type to be related to the differences in promotion energies for liafllum and titanium metal from their $\mathrm{d}^{2} \mathrm{~s}^{2}$ ground state to their $d^{3} s$ first excited state. They argue that the occurrence of unit $I$ as a metal P.C.P. in $\mathrm{Ti}_{2} \mathrm{~S}$ but not $\mathrm{Hf}_{2} \mathrm{~S}$ is related to the greater accessibility of the $d^{3}$ s state for titanium metal than for hafnium because of the difference in promotion energies.

$\mathrm{Ti}_{2} \mathrm{~S}$ and $\mathrm{Hf}_{2} \mathrm{~S}$ might be compared from the point of view that their structural differences are related to differences in the bonding contributions of titanium and hafnium metal. The hafnium P.C.P. In $\mathrm{Hf}_{2} \mathrm{~S}$ can be described as a trigonal anti-jrism formed by a triangular arrangement of three sulfur atoms above the central atom and a triangular arrangement of three hafnium atoms below the central atom (63). This polyhedron differs substantially from the metal P.C.P. observed for titanium atoms in the $\mathrm{TI}_{2} \mathrm{~S}$ structure. The sulfur P.C.P. also differ in that although sulfur is trigonal prismatically coordinated in $\mathrm{Hf}_{2} \mathrm{~S}$, the prisms do not have any of the metal capping atoms off the prism faces which is 
characteristic of the trigonal prisms in the $\mathrm{Ti}_{2} \mathrm{~S}$ phase. The lack of capping atoms in $\mathrm{Hf}_{2} \mathrm{~S}$ indicates that the bonding electrons of sulfur atoms are concentrated in bonds to the six hafnium atoms forming the trigonal prism, rather than to seven to nine atoms, as for the prisms of the $\mathrm{Ti}_{2} \mathrm{~S}$ structure. Perhaps the greater concentration of electrons from sulfur in the six Hf-S trigonal prismatic bonds in $\mathrm{Hf}_{2} \mathrm{~S}$ would require a smaller electron contribution from the metal atom for the same Hf-S bonds. If, indeed, hafnium does contribute less electron density than titanium to its Me-X bonds, the difference should be expressible in terms of $\psi_{\text {bond }}$ for the two metals.

$\psi_{\text {bond }}(H f)$ might be expressed as $n_{1}\left(d^{3}\right)_{1}+n_{2}\left(d^{2}\right)_{1-3-f o l d}$, where the $\left(d^{3}\right)_{1}$ term accounts for bond formation to the trigonal anti-prismatic arrangement of nelghbor atoms and its avaliability requires promotion to the $s d^{3}$ excited state. The second term also accounts for the bonding in the P.C.P. where the four d-orbital lobes of the $\left(d^{2}\right)$ orbitals, which would point to four of the six atoms of the anti-prism, are shared equally over all six atoms by the 3-fold symmetry axis of the anti-prism. Since the $\left(d^{2}\right)_{1}$ term corresponds to the ground state configuration while the $\left(d^{3}\right)_{1}$ term requires promotion to the $s d^{3}$ state, it might be expected that the $\mathrm{sd}^{3}$ contribution relative to the $\mathrm{d}^{2} \mathrm{~s}^{2}$ contribution is smaller in the bonding description for hafnium than in the bonding 
description for titanium in $\mathrm{TI}_{2} \mathrm{~S}$. This difference in relative contribution would be consistent with the difference in promotion energy and the Implication of the last paragraph that a smaller electron contribution from hafnium may be required in $\mathrm{Hf}_{2} \mathrm{~S}$ than would be required if the structure type corresponded to $\mathrm{TI}_{2} \mathrm{~S}$.

The $\mathrm{Hf}_{2} \mathrm{P}$ structure is isotypic with $\mathrm{Ti}_{2} \mathrm{~S}$, and its occurrence might be considered in the following way. A hypothetical $\mathrm{Hf}_{2} \mathrm{P}$ phase occurring with the $\mathrm{Hf}_{2} \mathrm{~S}$ structure type is not known and might be considered to be less stable than the known structure type because the phosphorus atom with one less electron than a sulfur atom would require that the metal atom make a larger contribution of electron density in the Hf-P bonds. The increase in electron contribution from the metal was related to an increase in the contribution from the $s d^{3}$ state relative to the $d^{2} s^{2}$ contribution. Hafnium in $\mathrm{Hf}_{2} \mathrm{~S}$ can provide the required electron concentration for its $\mathrm{H} f-\mathrm{S}$ bonds without requiring complete promotion to the $\mathrm{sd}^{3}$ excited state. In $\mathrm{Hf}_{2} \mathrm{P}$ the smaller number of electrons avallable for bonding from phosphorous, in effect, forces an increased degree of promotion to the $\mathrm{sd}^{3}$ exclted state to provide the needed electron density relative to that for $\mathrm{Hf}_{2} \mathrm{~S}$ indicating that the $\mathrm{Hf}_{2} \mathrm{P}$ structure type $\left(\mathrm{TI}_{2} \mathrm{~S}\right)$ is more stable. 
2. The $\mathrm{Cr}_{4} \mathrm{As}_{3}$ and $\mathrm{Nb}_{4} \mathrm{As}_{3}$ structure types

Of the 19 different structure types of the layered class the $\mathrm{Cr}_{4} \mathrm{As}_{3}$ and $\mathrm{Nb}_{4} \mathrm{As}_{3}$ structures are the most similar. The 1r structural similarity was observed independently but was also described by Berger (47) in his paper on the structure determination of $\mathrm{B}-\mathrm{V}_{4} \mathrm{As}_{3}\left(\mathrm{Nb}_{4} \mathrm{As} \mathrm{s}_{3}\right.$ structure type). The structures can be described in terms of a common unit formed by metal and arsenic atoms which pack in the same way for both phases.

Figures 17 and 18 illustrate the two different packing sequences for this structural unit in the $\mathrm{Cr}_{4} \mathrm{As}_{3}$ and $\mathrm{Nb}_{4} \mathrm{As}_{3}$ structure types. The predominant structural differences between the two phases occur along the solid horizontal lines in Figure 17 and the broken horizontal lines in Figure 18. It is only for atom positions in proximity to these lines where differences in the nature of the metal and nonmetal P.C.P. occur. All other atom positions of the two structures are basically identical.

Figure 19 illustrates the differences in the nature of the P.C.P. occurring along the horizontal lines of connection of the structural unit. Although the P.C.P. of $\mathrm{Nb}(5)$ and $\mathrm{Cr}(1)$ are both of type VIII, there is a marked difference in the geomctry of the two P.C.Y. Differences in the types of P.C.P. for the two phases occur only for the arsenic atom positions. Although the As(3) atom position of both phases 


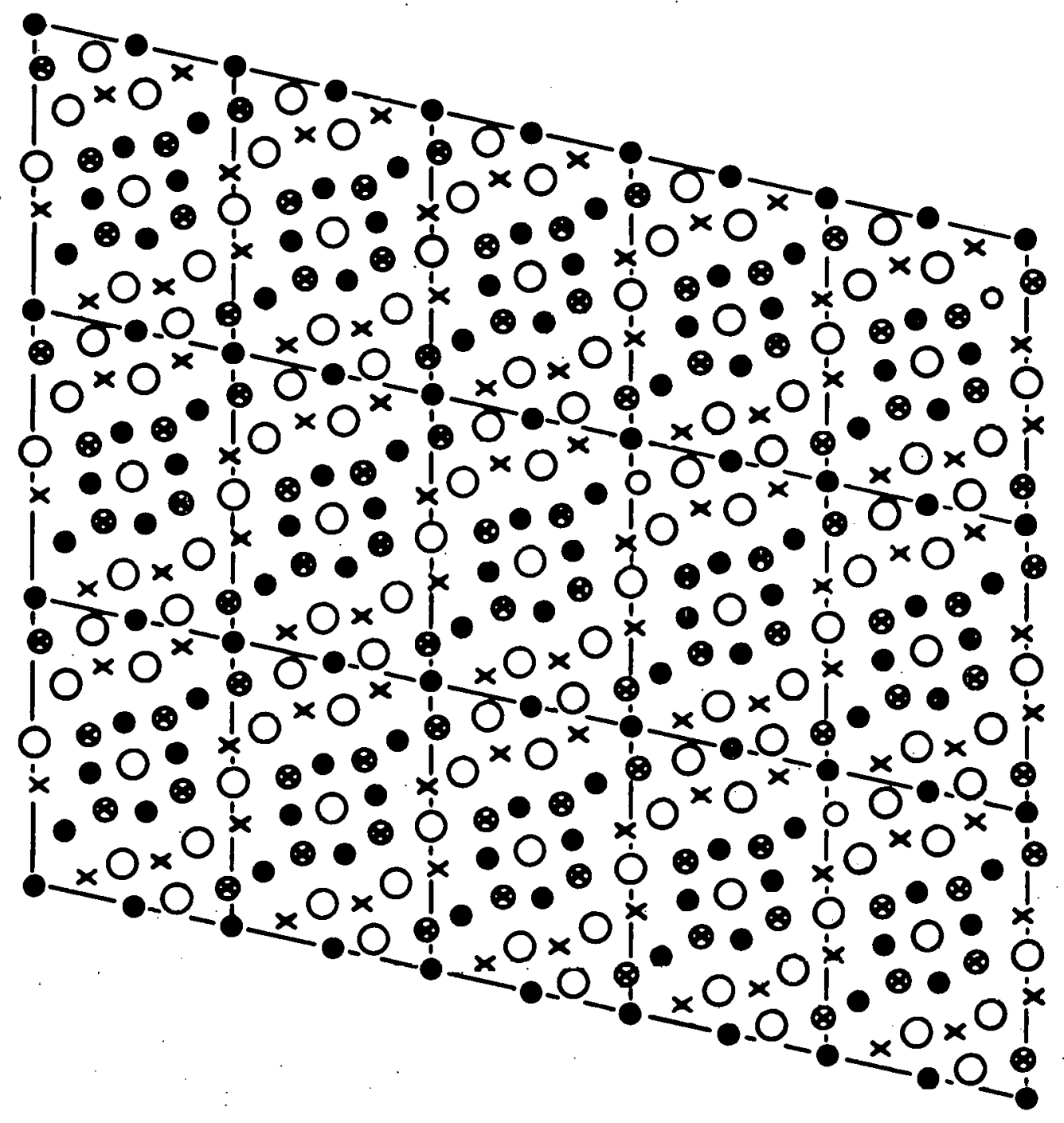

Figure 17. The $\mathrm{Cr}_{4} \mathrm{As}_{3}$ structure type. Solid lines indicate the unit cell 


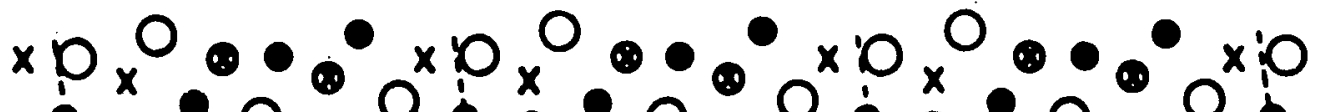

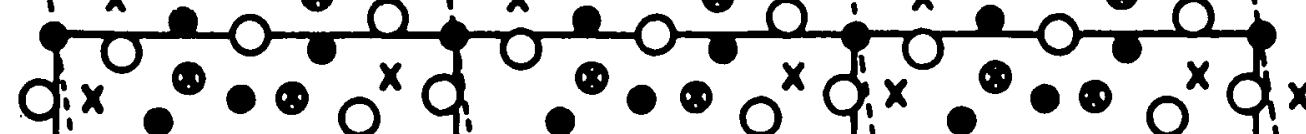

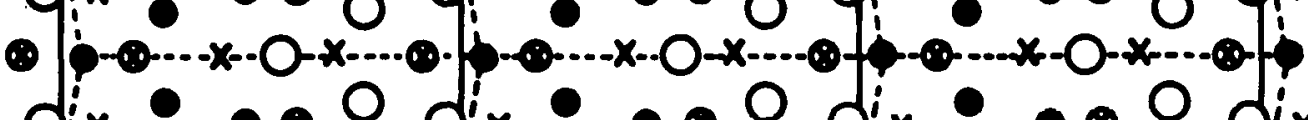

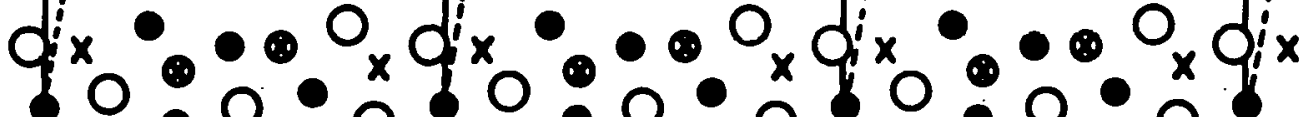

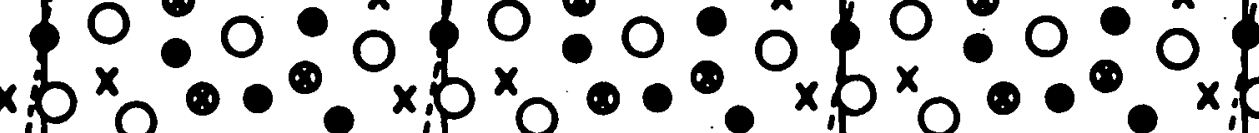

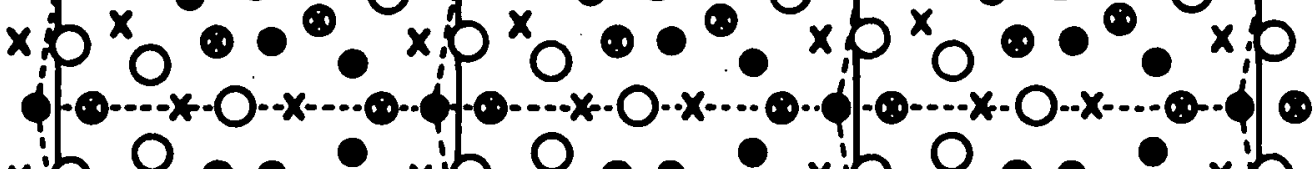
$\times b_{x}^{0} \cdot 0^{\circ} \times b^{0} \cdot 0 \times 0^{0} \cdot 0 \cdot 0$

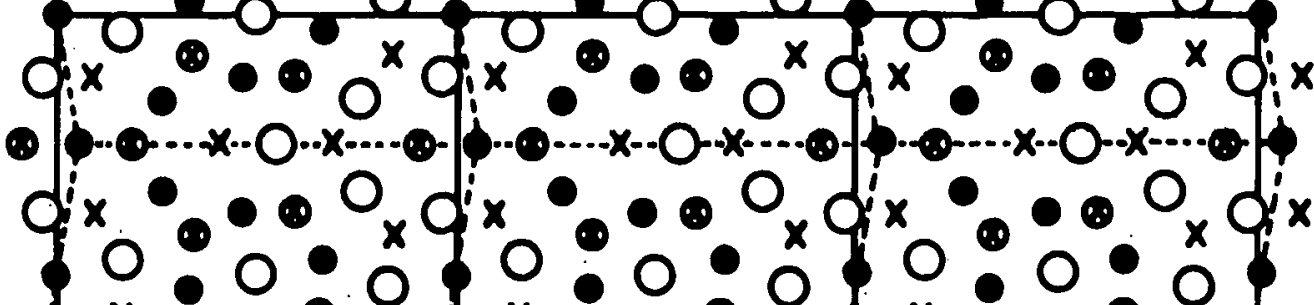
$x, 5 \times 0$ :

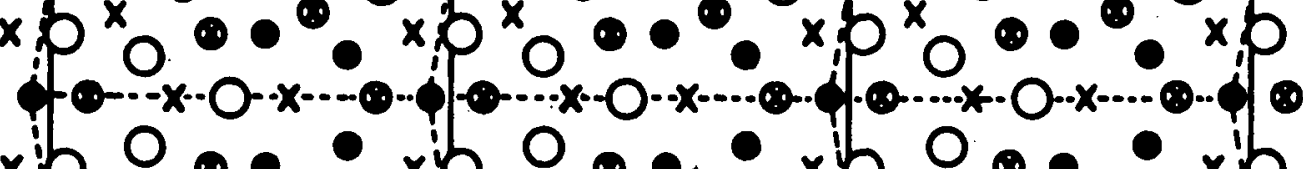

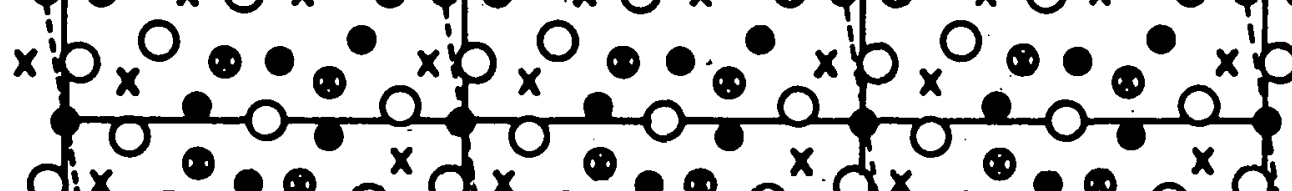

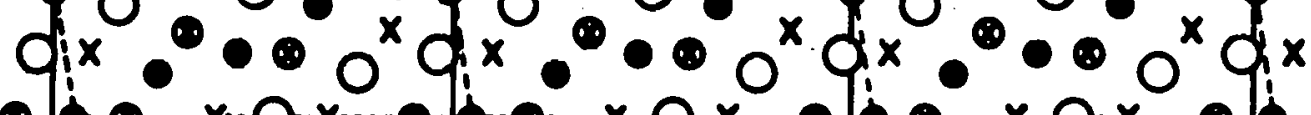

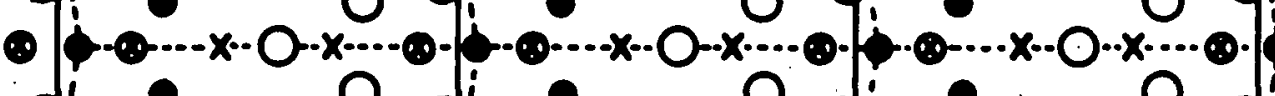
$d_{x}$

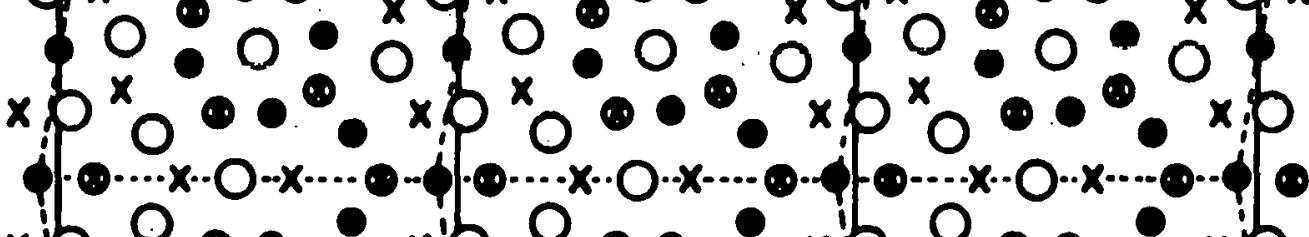

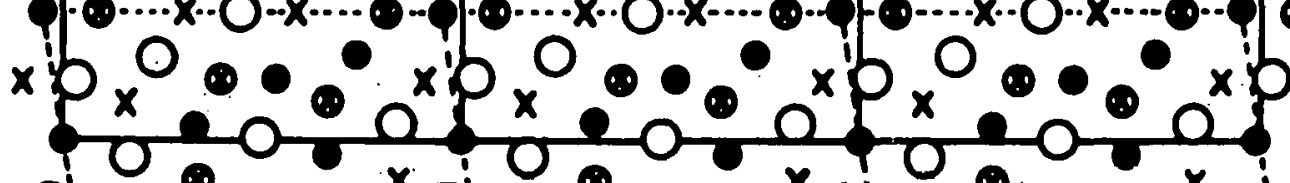

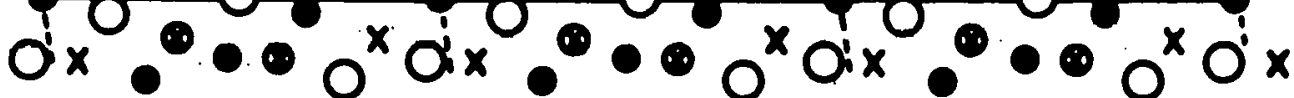

Figure 18. The solid lines indicate the $\mathrm{Nb}_{4} \mathrm{As}_{3}$ unit cell. Broken lines illustrate the packing of the $\mathrm{Cr}_{4} \mathrm{As}_{3}$ unit cell. to form the $\mathrm{Nb}_{4} \mathrm{As}_{3}$ structure type 

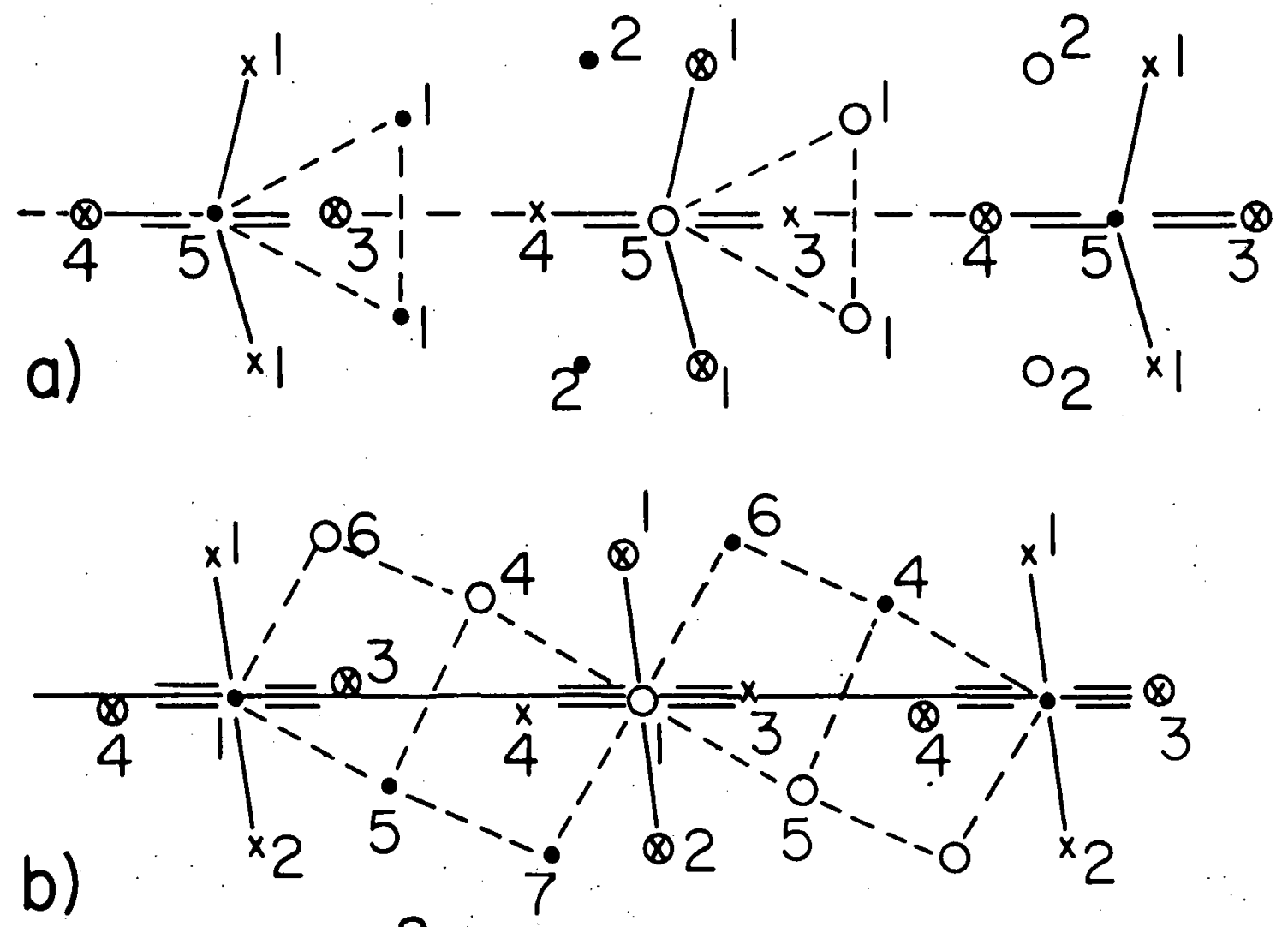

b)

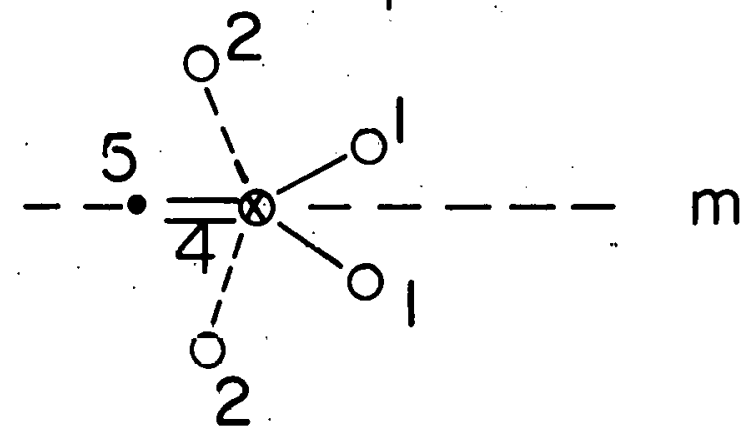

Figure 19. Differences in the nature of P.C.P. for $\mathrm{Nb}_{4} \mathrm{As}_{3}$ (part a) and $\mathrm{Cr}_{4} \mathrm{As}_{3}$ (part b) structure types along the horizontal Iines of connection of the structural un1t. Part c) 1llustrates the near neighbors for $\mathrm{As}(4)$ in $\mathrm{Nb}_{4} \mathrm{As}_{3}$ 
is trigonal prismatic, the two phases differ in the orientation of the prism. As(4) of $\mathrm{Cr}_{4} \mathrm{As}_{3}$ is also trigonal prismatic, while As(4) of $\mathrm{Nb}_{4} \mathrm{As}_{3}$ is the only nonmetal atom position in the structures of the layered class which is not trigonal prismatic. The coordination polyhedra of As(4) can be described as a highly distorted bi-capped tetrahedron. Within the framework of this bonding model, the occurrence of a P.C.P. for the As(4) atom which is not trigonal prismatic is thought to be related to the mm-point symmetry of the atomic coordination. The mm-point symmetry differs from the m-point symmetry found for most of the atom positions in the structures of this class. The presence of mm-point symmetry at As.(4) is not incompatible with the occurrence of trigonal prismatic coordination, since $\mathrm{As}(3)$ in $\mathrm{Nb}_{4} \mathrm{As}_{3}$ has $\mathrm{mm}-$ point symmetry and a trigonal prismatic P.C.P. Rather, it is felt the mm-point symmetry restriction combined with the seeming stability of the large grouping of metal P.C.P. which provides the framework within which the nature of the bonding of As(4) must be understood. It is difficult to envision any way in which As(4) could have trigonal prismatic coordination without changing the nature of the metal coordination polyhedra or the packing relationship between them.

The weakness of the structural model introduced here Iies in the emphasis on the metal contribution to the Me-X bonds. Although the contribution of the metal atom in bond 
formation is certainly important, it is the interaction between the metal and nonmetal atoms which is critical to the understanding of the structures discussed here. One of the Important questions concerning the nature of the Me-X bond interaction concerns the effect of the $\mathrm{x}$ bonding contribution on the metal bonding contribution and, of course, the effect of the metal bonding contribution on the nonmetal bonding contribution.

Iine discussion to this point has assumed that the nonmetal P.C.P. was trigonal prismatic. By assuming that the nonmetal P.C.P. was the same, the assumption was made that to a first approximation, the $X$ bonding contribution was insensitive to changes in the Me bonding contribution. Comparison of the $\mathrm{Nb}_{4} \mathrm{As}_{3}$ and $\mathrm{Cr}_{4} \mathrm{As}_{3}$ structure types indicate that a change in metal can influence the nonmetal P.C.P. even though the metal P.C.P. is not radically altered. The specific nature of this influence has not been determined. 
VIII. STRUCTURAL COMPARISONS

\section{A. Introduction}

Since each different structure type of the layered class can be viewed as a unique combination of several P.C.P., it will be the combination of P.C.P. that is the main topic of discussion in this section. By comparing two different structure types in a particular way, 1t becomes evident that certain arrangements of large numbers of atoms are common to different structures. Such a group of atoms will be referred to as a structural unit throughout the following discussion and will be described in terms of a unique combination of metal and nonmetal P.C.P. sharing faces and edges in the same way .

The purpose of comparing structures in terms of structural units is to help gain a better insight into how the similarities and differences between two structures might be related to similarities and differences in the chemical interactions between the various atoms that form the structures. Since the structures change dramatically with changing metal, nonmetal, Me/X ratio, etc., the comparisons made here attempt to limit some of the variables that effect the stability of a structure type. The comparison of different structures of stable compounds in the same Me-X. system, e.e.g., $\mathrm{TI}_{2} \mathrm{~S}$ and $\mathrm{TI}_{8} \mathrm{~S}_{3}$ or $\mathrm{Nb}_{21} \mathrm{~S}_{8}$ and $\mathrm{Nb}_{14} \mathrm{~S}_{5}$, suggests 
insight concerning the effect of changing Me/X ratio on structural stability within a particular Me/X system. Different systems exhibit different structural changes for a change in $\mathrm{Me} / \mathrm{X}$ ratio. By comparing two different phases with the same stoichiometry and nonmetal component but different metal component, the structural differences suggest information about, the difference in chemical interaction between different metal atoms and a particular nonmetal. An attempt will be made to correlate some of the structural differences for phases in the $\mathrm{Nb}-\mathrm{S}$ and Ti-S systems with the qualitative bonding considerations discussed previously.

$$
\text { B. } \mathrm{Ti}_{2} \mathrm{~S} \text { and } \mathrm{Ti}_{8} \mathrm{~S}_{3}
$$

The earlier discussion of the $\mathrm{TI}_{2} \mathrm{~S}$ and $\mathrm{Ti}_{8} \mathrm{~S}_{3}$ structures emphasized the P.C.P. present in each. The prominent structural feature of the two phases is the presence of the metal P.C.P. Of type I, which share faces and edges with the trigonal prisms about sulfur atoms. Figure 20a illustrates a structural unit for the T.1 ${ }_{2}$ s. structure which can be described as two P.C.P. of type I which share a common face, while each of the six remaining faces of the two fused cubes is shared with a face of a trigonal prism (1gnoring faces paraliel to the plane of the drawing). In Figure 21 , the stacking of this structural unit is 1llustrated for the $\mathrm{TI}_{2} \mathrm{~S}$ structure. Repetition of the unit in this fashion accounts 


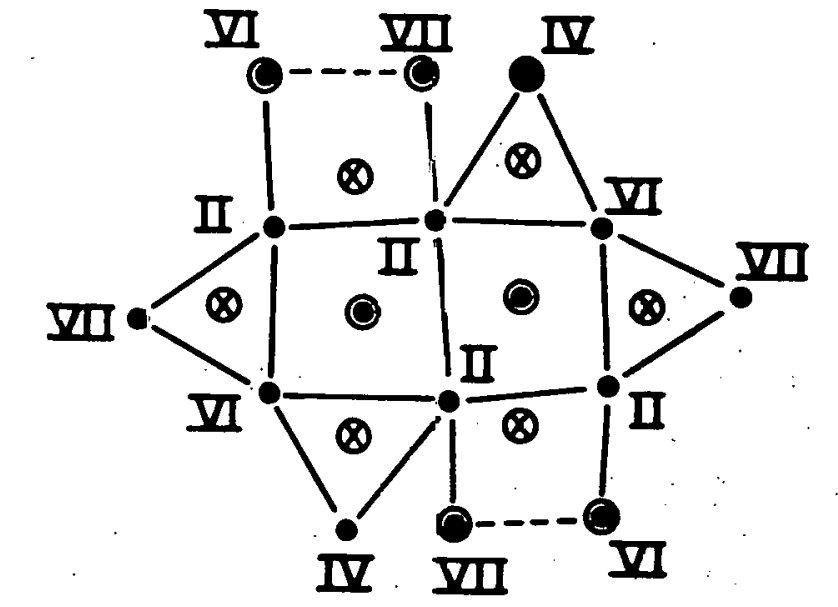

o)

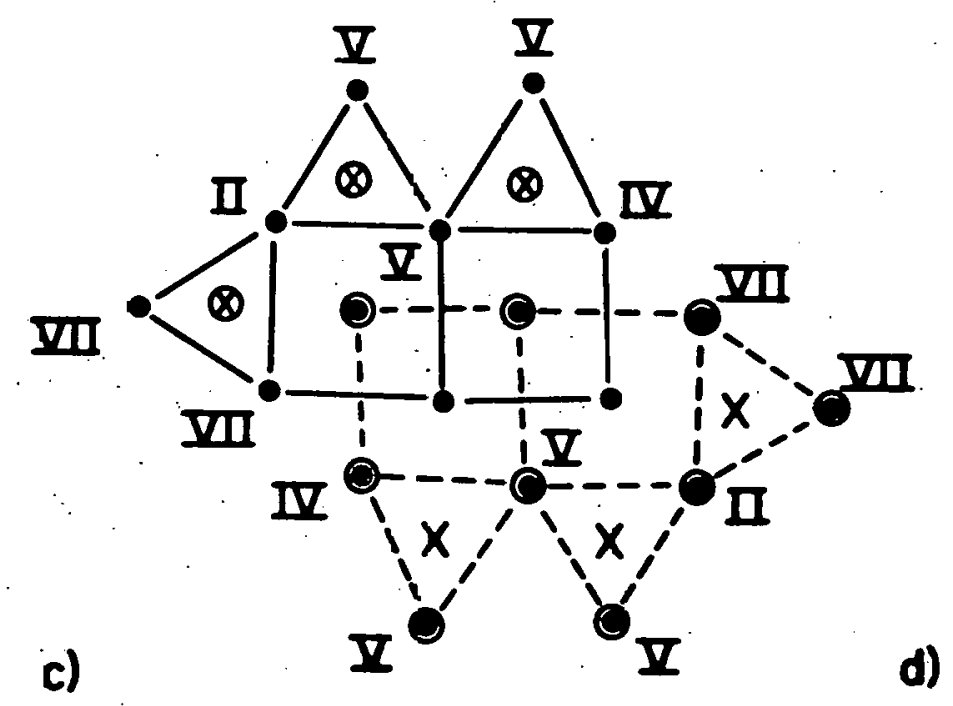

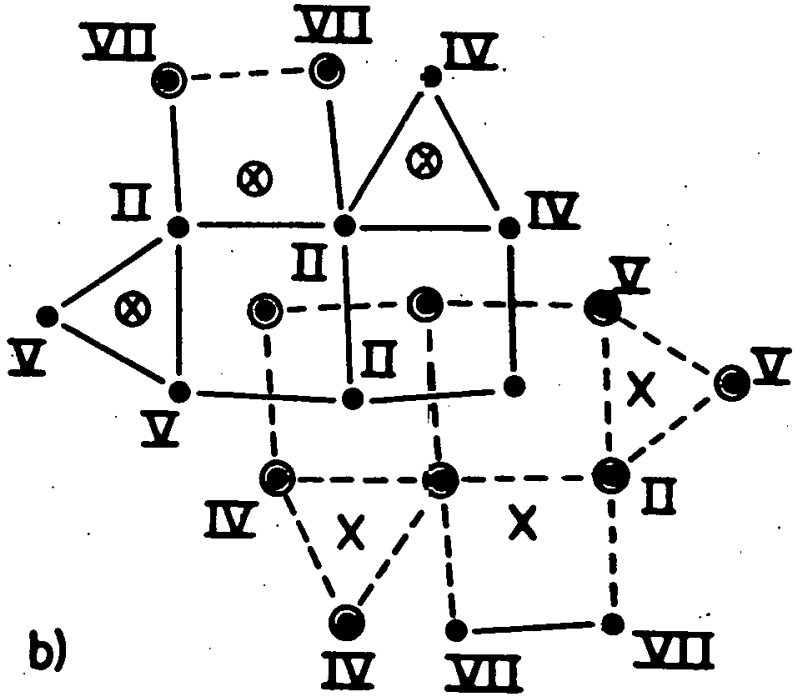

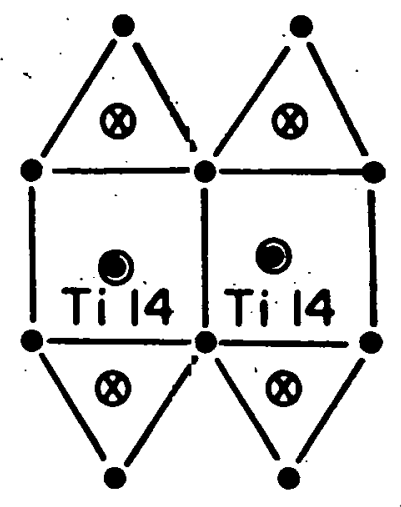

F1gure 20. The relationship between P.C.P. I and nonmetal trigonal prisms in $\mathrm{Ti}_{2} \mathrm{~S}$ and $\mathrm{Ti}_{8} \mathrm{~S}_{3}$. The types of P.C.P. for the poilyhedral metal atoms are indicated 
Figure 21. The $\mathrm{Ti}_{2} \mathrm{~S}$ structure as the packing of the structural unit in part a) of Figure 20. Solid lines illustrate P.C.P. centered about atoms in one layer of atom positions while broken lines correspond to P.C.P. for atom positions in the second layer 


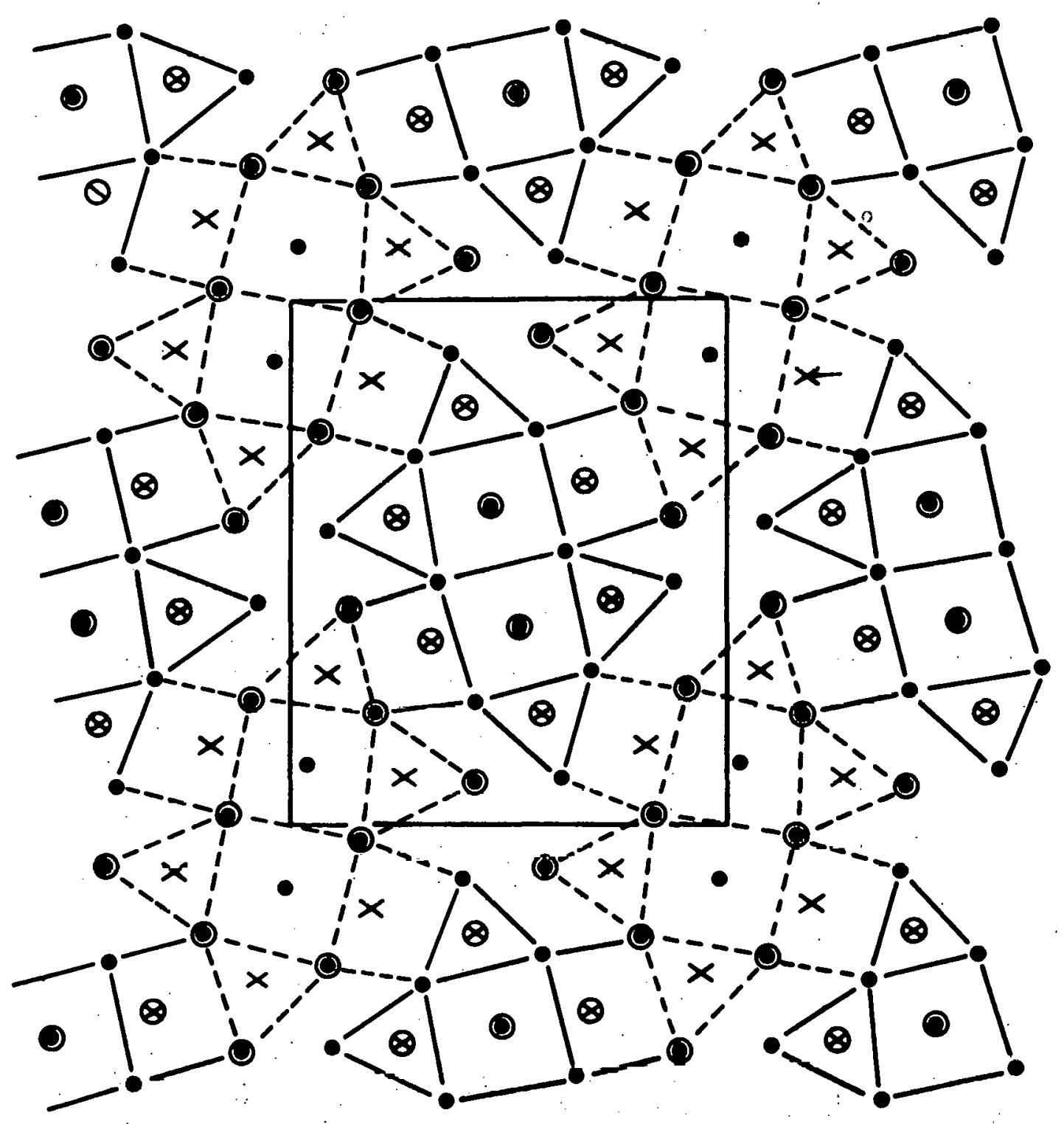


for all atoms of the $\mathrm{Ti}_{2} \mathrm{~S}$ structure. In Figure 21 , the $\mathrm{T} 1-\mathrm{T} 1$ edge of the $S(3)$ trigonal prism formed by $T 1(6)$ and $T 1(5)$ in one structural unit is also an edge of the trigonal prism about $S(1)$ in a neighboring structural unit. Edge sharing in this way illustrates the connections between the' structural units as they pack to form the $\mathrm{Ti}_{2} \mathrm{~S}$ structure.

Figure $20 \mathrm{~b}$ and $20 \mathrm{c}$ illustrate the two structural units of $\mathrm{Ti}_{8} \mathrm{~S}_{3}$ which are similar to the structural unit of $\mathrm{Ti}_{2} \mathrm{~S}$. Both of the $\mathrm{Ti}_{8} \mathrm{~S}_{3}$ units are formed from two P.C.P. of type I with a common face, but only three of the remaining six cube faces are shared with sulfur trigonal prisms. The presence of an inversion center in each of the two structural units relates the two halves of each structural unit, thus completing their description. The two units differ in the orientation of the prism axis for one of the three trigonal prisms. The unit in Figure $20 \mathrm{c}$ has all three prism axes perpendicular to the plane of projection, while the unit in part $b)$ of the figure contains $S(6)$ with a parallel prism axis. Figure 22 1llustrates the repetition of the two structural units in forming the $\mathrm{Ti}_{8} \mathrm{~S}_{3}$ structure. The unit in part b) of Figure 20 is outlined by solid lines, while the other unit is represented by broken lines. The same connection between units described for $\mathrm{Ti}_{2} 3$ in terms of the sharing of a trigonal prism edge between neighboring structural units also occurs $\operatorname{in} \mathrm{TI}_{8} \mathrm{~S}_{3} \cdot \mathrm{Ti}(13)$ and $\mathrm{TI}(15)$ form an 


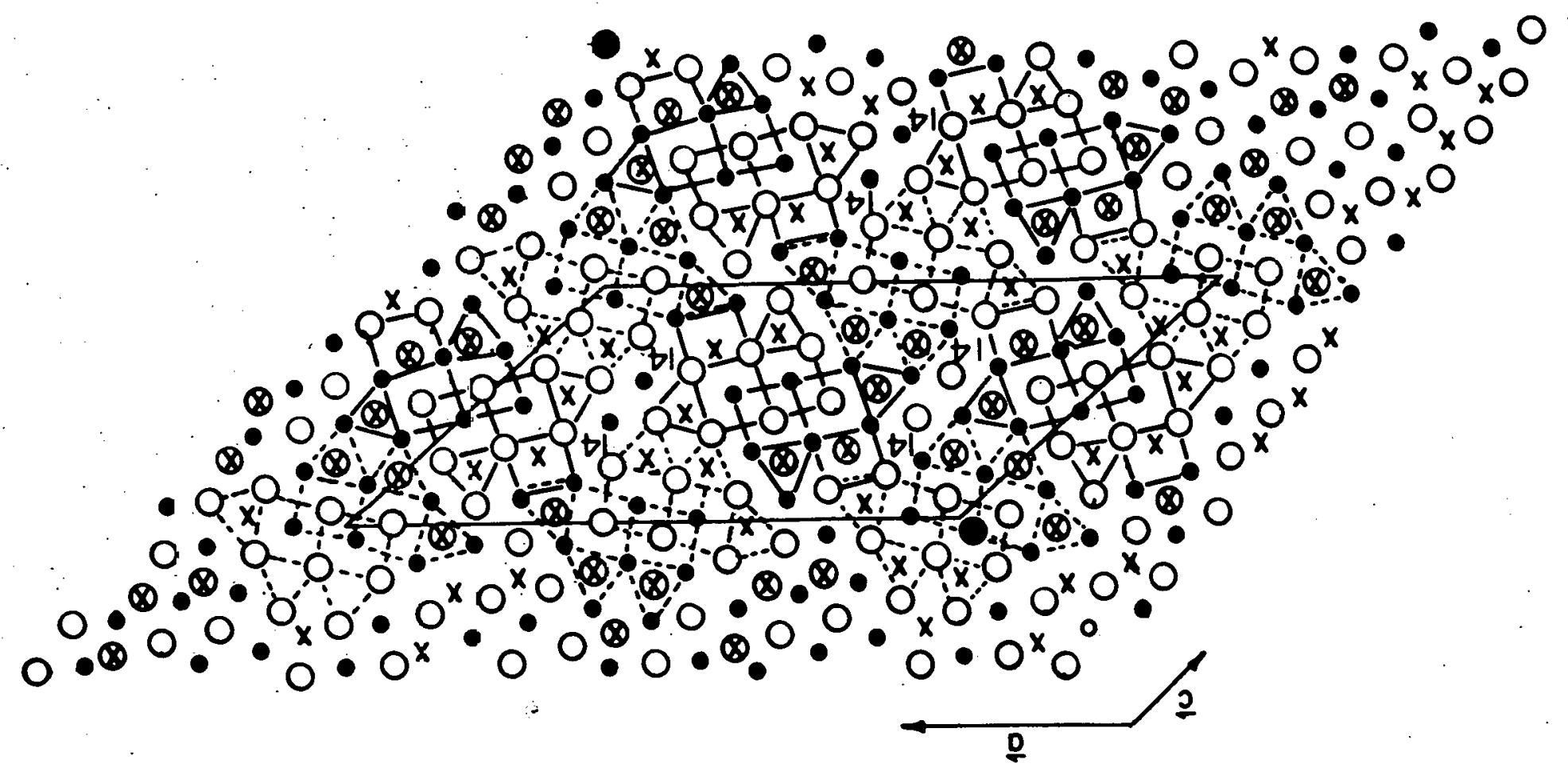

Figure 22. The $\mathrm{Ti}_{8} \mathrm{~S}_{3}$ structure as the stacking of the structural units in part b), c), and d) of Figure 20 
edge common to both the prism of $S(6)$ in one structural unit and the prism of $S(1)$ in the second unit. The repetition of these structural units as indicated in Figure 22 leaves a cubic hole which is filled by $\mathrm{T}(14)$, completing the structural description of $\mathrm{Ti}_{8} \mathrm{~S}_{3}$.

Viewing the $\mathrm{Ti}_{8} \mathrm{~S}_{3}$ structure in terms of the stacking of structural units similar to the structural unit in $\mathrm{Ti}_{2} \mathrm{~S}$ provides the simplification in the description of this complex phase. In comparing the $\mathrm{TI}_{2} \mathrm{~S}$ and $\mathrm{Ti}_{8} \mathrm{~S}_{3}$ structures with the other more metal-rich phases in this layered class, the structural units introduced here provide a basis for discussing the structural similarities and differences between the various structure types. Many of the structures in this class are characterlzed by the presence of P.C.P. Of type $I$ and the trigonal prisms of metal atoms about central nonmetal atoms. The structural units used to describe the $\mathrm{TI}_{2} \mathrm{~S}$ and $\mathrm{TI}_{8} \mathrm{~S}_{3}$ structures 1llustrate only one of the possible stacking arrangements of the metal cubes and trigonal prisms (metal cubes sharing faces with trigonal prisms). The structures of other phases are characterized by different stacking arrangements of cubes and prisms (e.g.g., $\mathrm{Nb}_{21} \mathrm{~S}_{8}$ and $\mathrm{Nb}_{14} \mathrm{~S}_{5}$ to follow).

Each of the structural untts in Figure 20 illustrates the face sharing of the metal cubes and trigonal prisms. The arrangement of sulfur atoms off the faces of the metal 
cubes is dependent upon, and can be related to, the type of P.C.P. found for those atoms which form the metal cubes" (P.C.P. I). That this is necessarily true can be seen by realizing that the sulfur atoms off the face of the cubes are part of the P.C.P. of those atoms which form the cube faces. This suggests a relationship between the P.C.P. for those atoms which form the edges of the cubes and the particular manner in which the cubes and prisms share faces In $\mathrm{Ti}_{2} \mathrm{~S}$ and $\mathrm{TI}_{8} \mathrm{~S}_{3}$. Figure 20 indicates that these metal atoms have P.C.P. which are predominantly of type II and V. This suggests that 1 it is the occurrence of P.C.P. II and V, which was previously associated with the use of $d_{1}^{2}$ bonding contribution, that is related to the particular arrangement of cubes and prisms in both $\mathrm{TI}_{2} \mathrm{~S}$ and $\mathrm{TI}_{8} \mathrm{~S}_{3}$.

Figure 20 also indicates the type of P.C.P. for those metal atoms which are part of the structural units but which are not polyhedral atoms of the cubes. The complete P.C.P. of many of the atoms of the structural unit are not specified until the stacking of the structural units is known. Figure 21 indicates the spacial relationship between the structural unit in Figure 20a and 1ts neighboring units, 1llustrating that the incomplete P.C.P. for the atoms at the extremities of the structural unit are completed by atoms in nelghboring units. Similar considerations apply for the structural units of $\mathrm{TI}_{8} \mathrm{~S}_{3}$. There is obviously a very important interdependence between the stacking of the structural units and the 
type of P.C.P. of the atoms at the edge of the structural units. The similarity in stacking of the structural units can thus be related (Figure 20) to the similarity in the types of P.C.P. for atoms at the extremity of the various structural units.

In the comparison of $\mathrm{Ti}_{2} \mathrm{~S}$ and $\mathrm{TI}_{8} \mathrm{~S}_{3}$, it is the type of P.C.P. for those atoms forming the extremities of the structural units that changes very little between the two phases (exemplified by similar packing of structural units in $\mathrm{Ti}_{2} \mathrm{~S}$ and $\left.\mathrm{Ti}_{8} \mathrm{~S}_{3}\right)$. Rather, it is the difference in the nature of the structural units themselves which 1llustrates the difference in the $\mathrm{Ti}_{2} \mathrm{~S}$ and $\mathrm{Ti}_{8} \mathrm{~S}_{3}$ structures. The difference in stoichiometry can be related to an increase in the number of P.C.P. of type $\mathrm{I}$ and $\mathrm{IV}$ in $\mathrm{Ti}_{8} \mathrm{~S}_{3}$ relative to $\mathrm{Ti}_{2} \mathrm{~S}$. The occurrence of both P.C.P. has been related to the use of $\mathrm{d}^{3} \mathrm{~s}$ hybrid orbital contribution in $\psi_{\text {bond }}(\mathrm{Me})$, which for titanium requires promotion to the $\mathrm{d}^{3} \mathrm{~s}$ ( $19 \mathrm{kcal} / \mathrm{mole}$ ) excited state. In contrast, the occurrence of P.C.P. II and V for the atoms forming the cube edges in both structures (corresponding to the same basic arrangement of cubes and prisms in the two structures) was related to a strong $d_{i}^{2}$ bonding contribution in $\psi_{\text {bond }}(\mathrm{Me})$, which for titanium corresponds to a larger relative bonding contribution from the $\mathrm{d}^{2} \mathrm{~s}^{2}$ ground state. In the comparison of the $\mathrm{Ti}_{8} \mathrm{~S}_{3}$ and $\mathrm{Nb}_{21} \mathrm{~S}_{8}$ structures to follow, an attempt will be made to relate the structural differences, expressed as differences in structural units 
and stacking of structural units, to differences in $\psi_{\text {bond }}$ for the different metals. The differences in $\psi_{\text {bond }}$ will. in turn be related to differences in the low lying electronic states of the metals.

$$
\text { C. } \mathrm{Nb}_{21} \mathrm{~S}_{8} \text { and } \mathrm{Nb}_{14} \mathrm{~S}_{5}
$$

The comparison of the $\mathrm{Nb}_{14} \mathrm{~S}_{5}$ and $\mathrm{Nb}_{21} \mathrm{~S}_{8}$ structures is difficult because of the relative complexity of both phases as can be seen In Figures 24 and 26. Details of the structural comparison will be given, since the same simple procedures can be applied in the comparisons of other structure types in the layered class. For the 19 different structure types in this class 210 such comparisons would be possible.

Figure 23 illustrates the two orientations of the structural unit that will be used to describe the structure of both $\mathrm{Nb}_{21} \mathrm{~S}_{8}$ and $\mathrm{Nb}_{14} \mathrm{~S}_{5}$. The structure units were found by simply superimposing scaled drawings of the two structures and finding the largest group of atoms that were common to both phases. The two orientations of the unit are ldentical in their relative placement of atoms and are related by the mirror plane represented by the broken line separating part a) and part b) of the drawing. This structural unit, formed by a unique combination of cubes and trigonal prisms, can be used to describe the two structures emphasizing their structural similarities. 
Figure 23. The structural units common to the $\mathrm{Nb}_{21} \mathrm{~S}_{8}$ and $\mathrm{Nb}_{14} \mathrm{~S}_{5}$ structure types. A.toms in the two structural units which have different P.C.P. are identified by Roman numerals corresponding to the type of P.C.P. for the appropriate atoms 


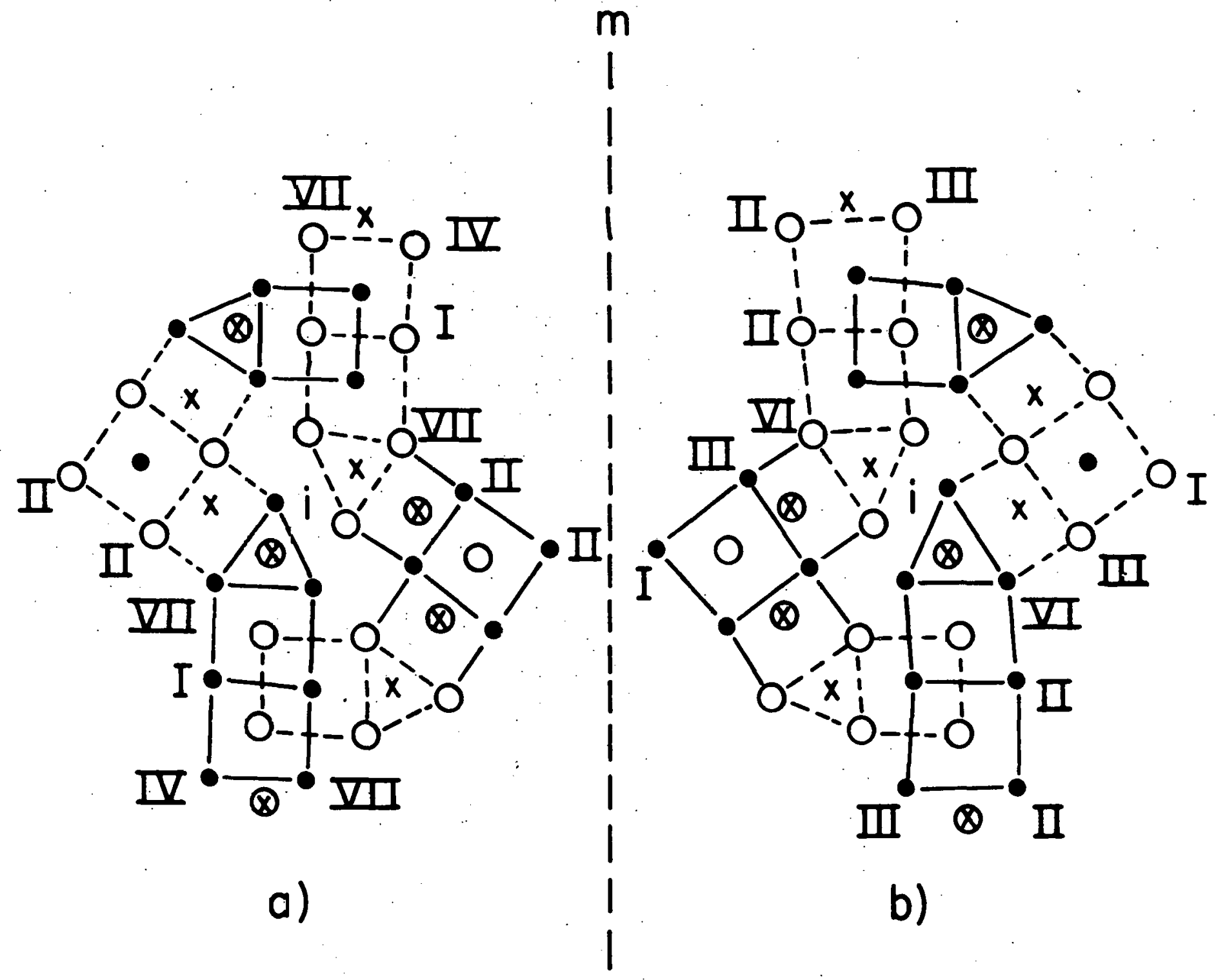


The repetition of the structural unit with the orientation indicated in part a) of Figure 23 by the translation vectors of the unit cells of $\mathrm{Nb}_{21} \mathrm{~S}_{8}$ and $\mathrm{Nb}_{14} \mathrm{~S}_{5}$ is indicated in Figures 24 and 26, respectively. The relative orientation of each of the unit cells as well as all the atoms within each unit cell are indicated in the drawings. Obviously, not all of the atoms for either phase are accounted for by the axial translations of the structural unit. The number of atoms between the units drawn is noticeably larger for $\mathrm{Nb}_{14} \mathrm{~S}_{5}$ than for $\mathrm{Nb}_{21} \mathrm{~S}_{8}$ corresponding to the larger unit cell of $\mathrm{Nb}_{14} \mathrm{~S}_{5} \cdot$. Most of these atoms are accounted for by a second repetition of the same structural unit in the two respective unit cells.

Figure 25 illustrates the manner in which the structural unit is repeated in forming the $\mathrm{Nb}_{21} \mathrm{~S}_{8}$ structure. The structural units which were indicated in Figure 24 are also represented in Figure 25 and are outlined with broken lines. The drawing illustrates that there are a number of atoms which are common to the two structural units. In Figure 27 the same type of drawing is given, but it is the orientation of the structural unit in Figure $23 \mathrm{~b}$ which is indicated by the solid lines. For $\mathrm{Nb}_{14} \mathrm{~S}_{5}$ the overlap of the structural units is less than the overlap in $\mathrm{Nb}_{21} \mathrm{~S}_{8}$. Repetition of the structural unit for $\mathrm{Nb}_{14} \mathrm{~S}_{5}$ does not account for all of the atoms in the unit cell. The two independent niobium 


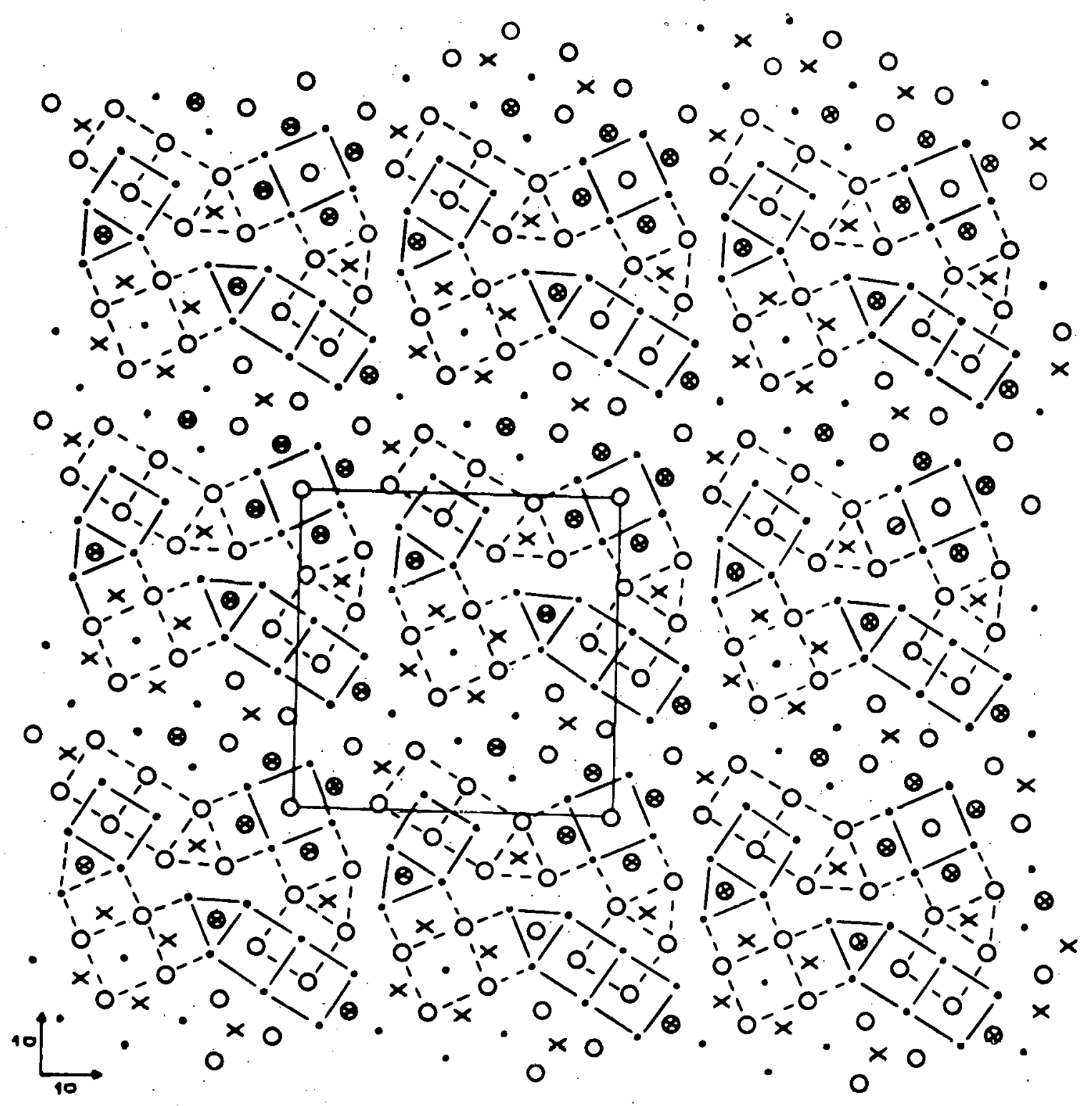

Figure 24. $\mathrm{Nb}_{21} \mathrm{~S}_{8}$ structure type lllustrating the structure unit of part a) in Figure 23.. The unit cell is indicated 


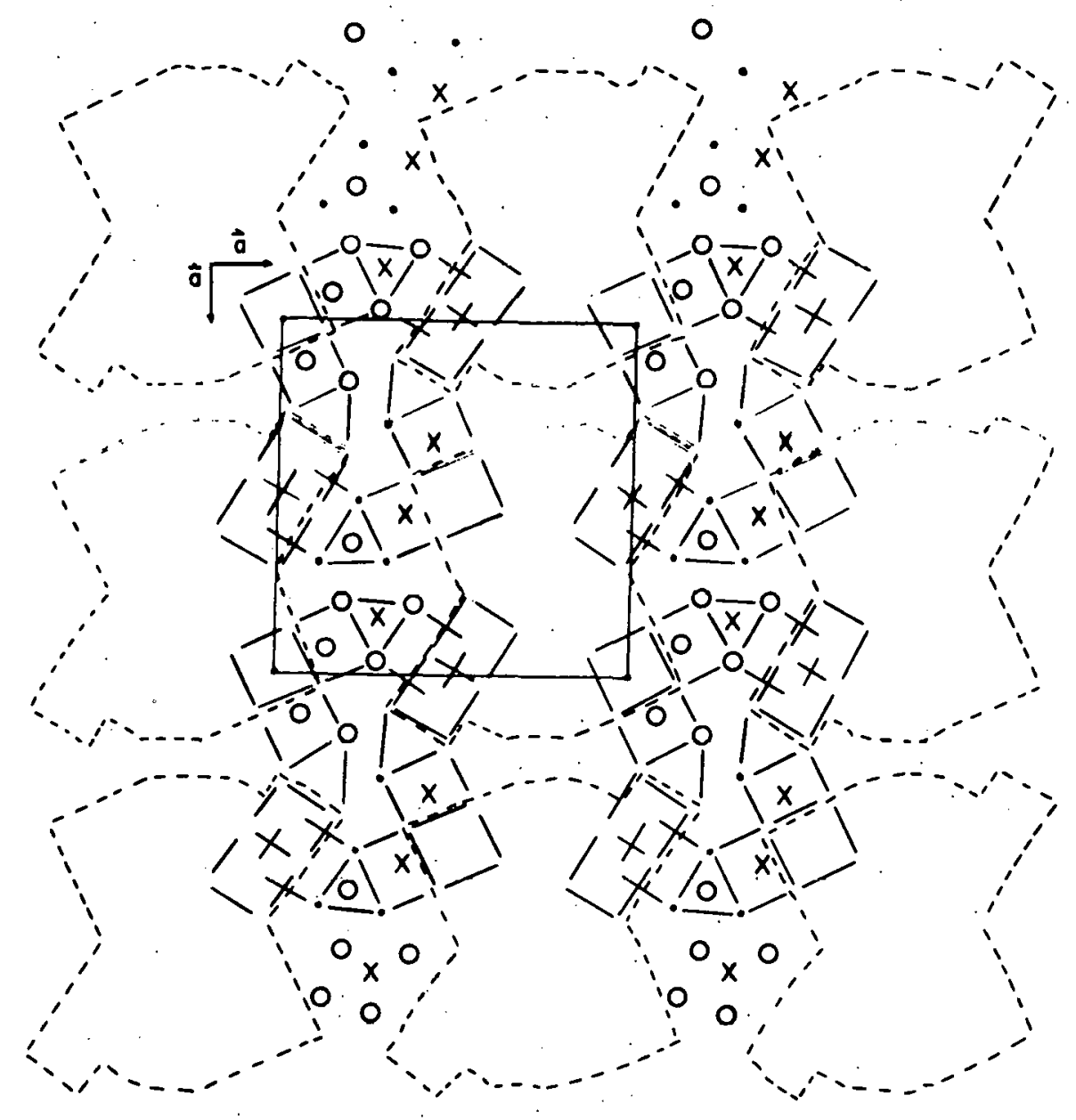

The location of the structure unit in Figure 24 is sliuwn by the urokerl lines whlle the sulid liries represent the repetition of the structure unit (part a) of Figure, 23 .

Figure 25. $\mathrm{Nb}_{21} \mathrm{~S}_{8}$ structure type 


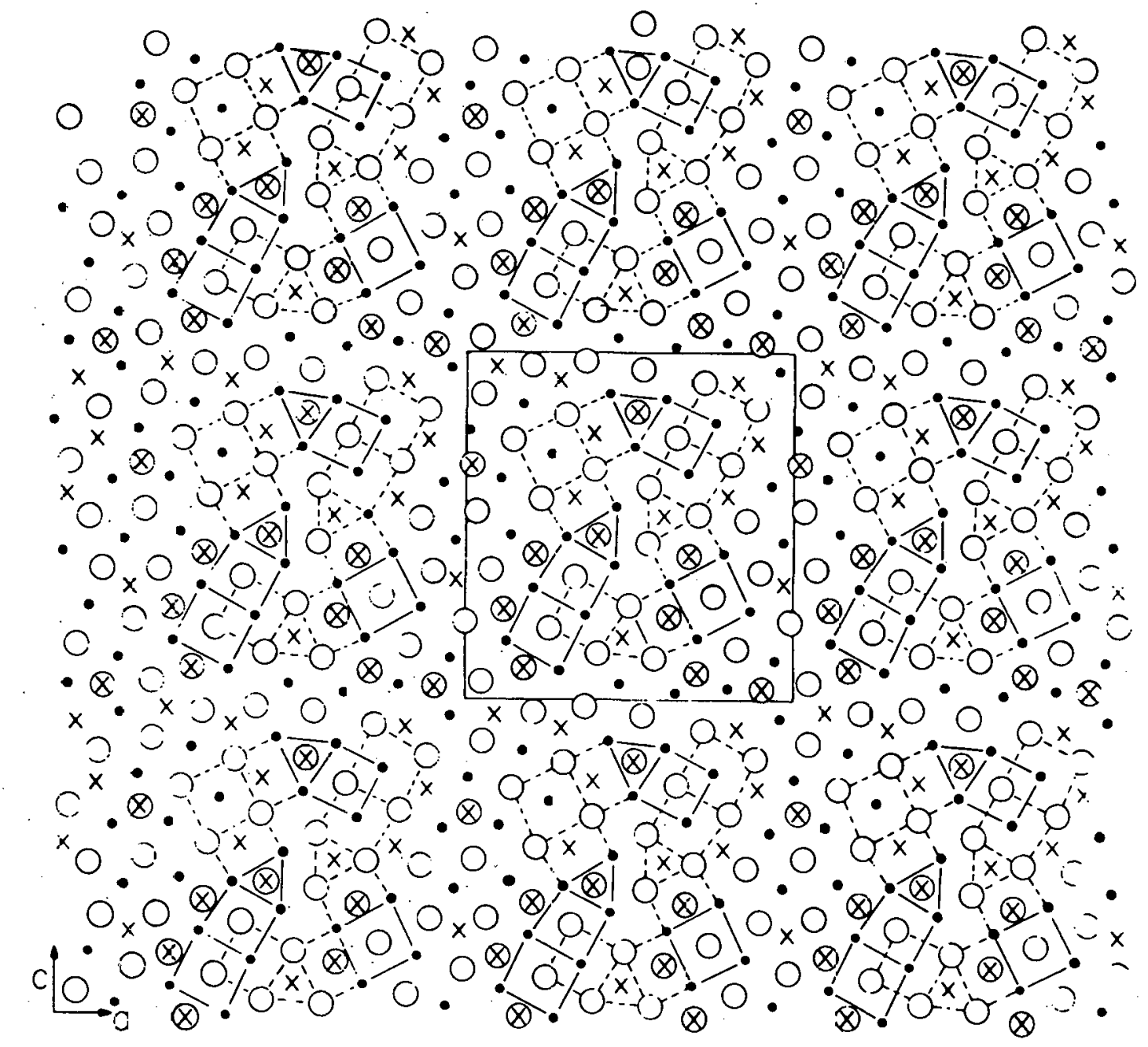

Figure 25. $\mathrm{Nb}_{14} \mathrm{~S}_{5}$ structure type. The structural unit in part a) of Figure 23 is indicated. The unit cell is outlined by solid lines 
positions that are not part of the structural unit have P.C.P. of type I and IV as indicated in Figure 27. The metal contribution to the bonds for both of these P.C.P. was previously described in terms of a $\left(\mathrm{sa}^{3}\right)_{1}$ ho combination which is available in the $\mathrm{sd}^{4}$ niobium ground state.

Comparing $\mathrm{Nb}_{21} \mathrm{~S}_{8}$ and $\mathrm{Nb}_{14} \mathrm{~S}_{5}$ in terms of this large structural unit indicates that their similarity transcends the earlier description of the structures in terms of their simflar P.C.P. Not only are the P.C.P. similar in both phases, but the unit of 44 atoms corresponds to a large number (24) of P.C.P. which share faces and edges in exactly the same way. Recalling the large number of possible ways that the various P.C.P. can stack to fill space and noting the recurrence of the large unit in both structures suggests that the structural unit is a particularly stable entity in the $\mathrm{Nb}-\mathrm{S}$ system.

The difference in the stacking of this structural unit in $\mathrm{Nb}_{14} \mathrm{~S}_{5}$ and $\mathrm{Nb}_{21} \mathrm{~S}_{8}$ is closely related to the differences in the P.C.P. at the edges of the structural units in the two phases. Figure 23 indicates which type of P.C.P. is found for the atoms along the edge of the units which exhibit different P.C.P. in the two structures. It is interesting that. while the P.C.P. at the edge of the units change, the basic identity of the unit is not altered. This is in contrast to the comparison of $\mathrm{Ti}_{2} \mathrm{~S}$ and $\mathrm{Ti}_{8} \mathrm{~S}_{3}$ where it was 


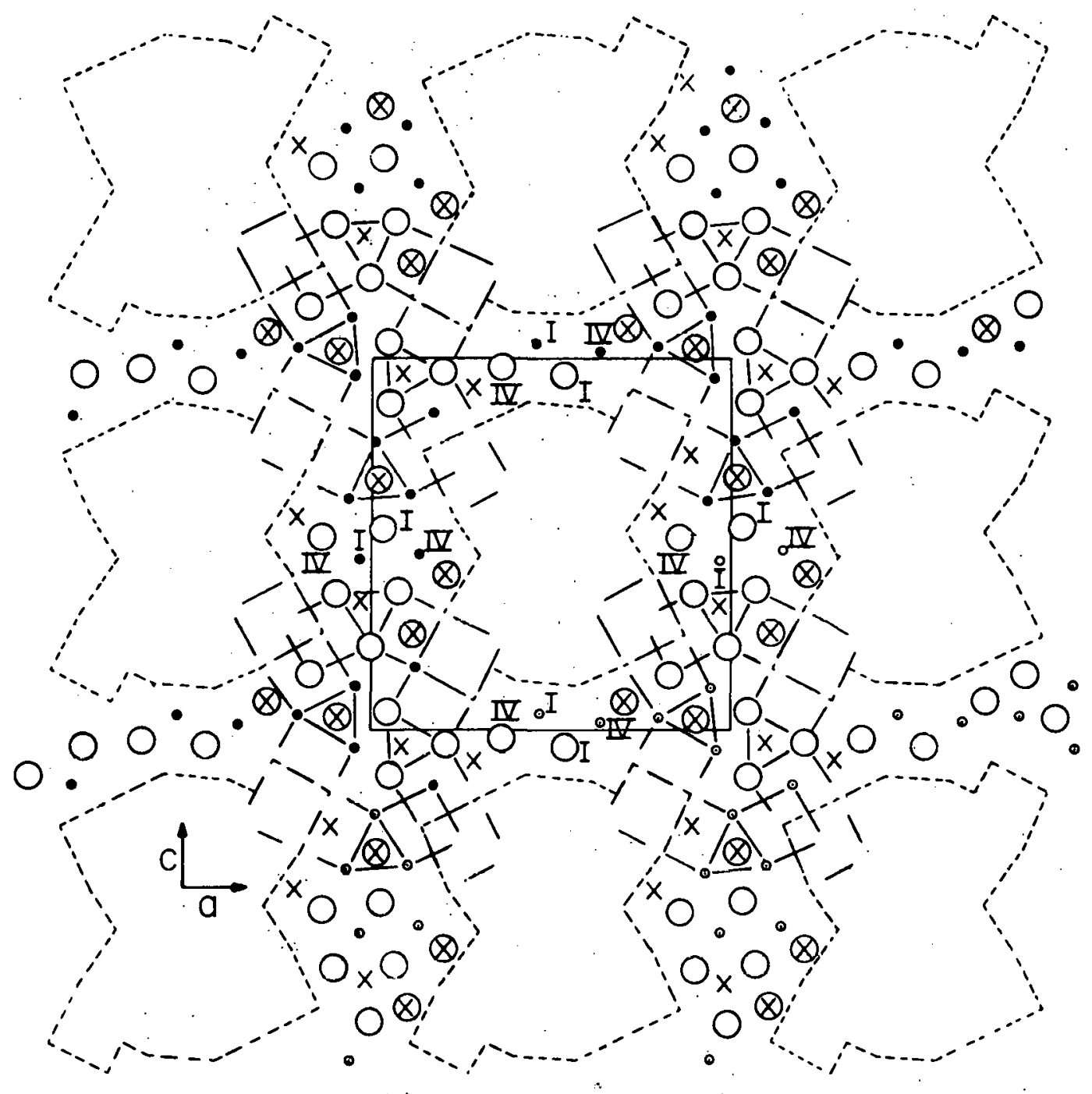

The structural unit in Figure 26 is represented by broken lines. Solid lines illustrate the location with $\mathrm{Nb}_{74} \mathrm{~S}_{5}$ of the structural unit in part. b) of Figure 23. Atom positions of the structure which are not part of efither structural unit are identified by their P.C.P.

Figure 27. ${ }^{\mathrm{Nb}} 14 \mathrm{~S}_{5}$ 
pointed out that the P.C.P. at the edges of the structural units changed very little while the units themselves differed.

$$
\text { D. } \quad \mathrm{Nb}_{21} \mathrm{~S}_{8} \text { and } \mathrm{Ti}_{8} \mathrm{~S}_{3}
$$

A comparison of the $\mathrm{Nb}_{21} \mathrm{~S}_{8}$ and $\mathrm{Ti}_{8} \mathrm{~S}_{3}$ structures provides an ideal case for considering a possible relationship between their structural differences and the different metal bonding contribution of the nloblum and titanium atoms. The $\mathrm{Me} / \mathrm{X}$ ratio of 2.667 for $\mathrm{Ti}_{8} \mathrm{~S}_{3}$ and 2.625 for $\mathrm{Nb}_{21} \mathrm{~S}_{8}$ are very nearly equal, which is important considering the structural. differences (lattice parameter, space group symmetry, number of each type of P.C.P., etc.) between $\mathrm{Nb}_{21} \mathrm{~S}_{8}$ and $\mathrm{Nb}_{14} \mathrm{~S}_{5}$ despite a relatively small difference in stoichiometry. The presence of the same nonmetal component in both phases is also important, since structures often vary considerably with a change in the nonmetal, $\underline{\mathrm{e}} \cdot \underline{\mathrm{g}}$., $\mathrm{Hf}_{2} \mathrm{P}$ and $\mathrm{Hf}_{2} \mathrm{~S}, \mathrm{Fe}_{2} \mathrm{P}$ and $\mathrm{Fe}_{2} \mathrm{As}, \mathrm{Ta}_{2} \mathrm{P}$ and $\mathrm{Ta}_{2} \mathrm{~S}$, etc. It might be expected that the structural differences between $\mathrm{Nb}_{21} \mathrm{~S}_{8}$ and $\mathrm{Ti}_{8} \mathrm{~S}_{3}$ are related more to the difference in the metal contribution to the $\mathrm{Nb}-\mathrm{S}$ and Ti-S bonds than to any other variable.

The structures of both phases were discussed above, and emphasis was placed on the occurrence of metal cubes and prisms in illustrating the common features of the two structures. The structural differences can be related to 
differences in the particular arrangement of cubes and prisms in the two structures. Differences in the arrangement of cubes and prisms can in turn be related to the observed differences in the types of P.C.P. for those atoms which are in the polyhedral positions of the metal cubes.

Figure 28 illustrates two similar structural units for $\mathrm{Nb}_{21} \mathrm{~S}_{8}$ and $\mathrm{Ti}_{8} \mathrm{~S}_{3}$. Each unit is formed by four metal P.C.P. of type I and four trigonal prisms. The two units differ in the relative orientation of the cubes and prisms. The figure also 1llustrates the nature of the P.C.P. of those atoms which form the cubes and prisms indicating the differences in the relative number of P.C.P. V and VII between the two phases.

Although the different structural units were used to compare $\mathrm{Ti}_{2} \mathrm{~S}$ with $\mathrm{Ti}_{8} \mathrm{~S}_{3}$ and $\mathrm{Nb}_{21} \mathrm{~S}_{8}$ with $\mathrm{Nb}_{14} \mathrm{~S}_{5}$, the units in Figure 27 could also have been used to describe the $\mathrm{Ti}_{8} \mathrm{~S}_{3}$ and $\mathrm{Nb}_{21} \mathrm{~S}_{8}$ structures. The choice of a particular structural unit is certainly not unique, but is dependent upon the particular comparison being made. The choice of units in this comparison emphasizes the differences in cube and prism orientation while identifying which particular metal atoms have different P.C.P. In the two structures.

A comparison of the total number of each type of P.C.P. present in the two phases accentuates the structural similarities and differences between $\mathrm{Nb}_{21} \mathrm{~S}_{8}$ and $\mathrm{T} 1_{8} \mathrm{~S}_{3} \cdot$ The 


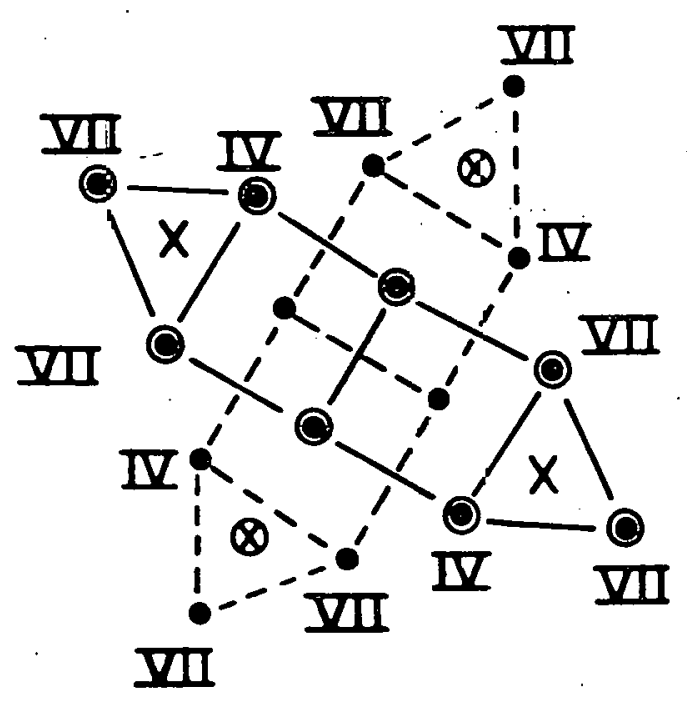

$\mathrm{Nb}_{21} \mathrm{~S}_{8}$

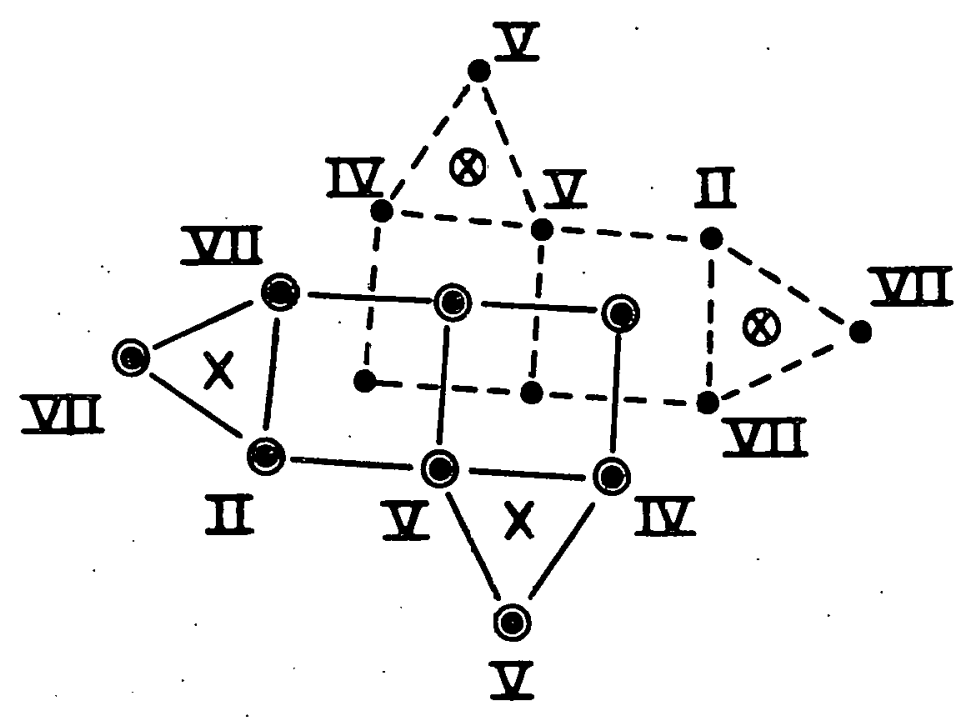

$\mathrm{Ti}_{8} \mathrm{~S}_{3}$

\footnotetext{
Figure 28. The different relationship between the metal P.C.P. of type I and the nonmetal trigonal prisms in $\mathrm{Nb}_{21} \mathrm{~S}_{8}$ and $\mathrm{Ti}_{8} \mathrm{~S}_{3}$. The types of metal P.C.P. are indicated
} 
number of P.C.P. calculated corresponds to the integral. number of unit cells of each phase that contains the same number of sulfur atoms. The numbers listed in Table 17 correspond to three unit cells of $\mathrm{Nb}_{21} \mathrm{~S}_{8}$ and two unit cells of $\mathrm{Ti}_{8} \mathrm{~S}_{3}$. The difference of two in the total number of metal atoms for the two phases accentuates the small difference in stoichionetry. The $\mathrm{Nb}_{21} \mathrm{~S}_{8}$ structure contains 32 more P.C.P. of type VII than $\mathrm{Ti}_{8} \mathrm{~S}_{3}$, while $\mathrm{Ti}_{8} \mathrm{~S}_{3}$ contains ten more P.C.P. of type $I$ and 24 more of type $V$. (The number of all other types of P.C.P. are the same.)

Table 17. Numerical comparison of the types of P.C.P. in $\mathrm{Ti}_{8} \mathrm{~S}_{3}$ and $\mathrm{N}_{21} \mathrm{~S}_{8}$

Number of Atoms with P.C.P.

\begin{tabular}{|c|c|c|c|c|c|c|c|}
\hline \multirow[b]{2}{*}{ Phase } & & & & & & \multirow{2}{*}{$\begin{array}{l}\text { Total } \\
\text { Metal }\end{array}$} & \multirow{2}{*}{$\begin{array}{l}\text { Total } \\
\text { Sulfur }\end{array}$} \\
\hline & $I$ & II & IV & V & VII & & \\
\hline $\mathrm{Ti}_{8} \mathrm{~S}_{3}$ & 40 & 24 & 24 & 24 & 16 & 128 & 48 \\
\hline $\mathrm{Nb}_{21} \mathrm{~S}_{8}$ & 30 & 24 & $24:$ & 0 & 48 & 126 & 48 \\
\hline
\end{tabular}

A simple calculation indicates that $25 \%$ of the polyhedra atoms for the cubes in $\mathrm{Nb}_{21} \mathrm{~S}_{8}$ are of type II or $\mathrm{V}$ and $25 \%$ are of type VII. For $\mathrm{Ti}_{8} \mathrm{~S}_{3}, 46.7 \%$ of the polyhedral atoms are of type II or $V$, while only $6.67 \%$ are of type VII. 
The difference in the relative number of P.C.P. VII and V for the two phases can be related to the type of P.C.P. found for the atoms which are polyhedrai atoms for the cubic units of metal atoms.

Understanding of the structural difference between $\mathrm{Ti}_{8} \mathrm{~S}_{3}$ and $\mathrm{Nb}_{21} \mathrm{~S}_{8}$ depends on understanding why the tendency to form P.C.P. of type VII is greater for niobium metal in $\mathrm{Nb}_{21} \mathrm{~S}_{8}$ than for titanium in $\mathrm{Ti}_{8} \mathrm{~S}_{3}$. The occurrence of unit $\mathrm{V}$ was related to the $\left(\mathrm{d}^{2}\right)_{i}$ contribution of the central atom in forming its Me-X bonds. In discussing the Me-X bond formation in unit VI and VII, the contribution of the central metal atoms represented by the ho's of $n_{1}\left(d^{2}\right)_{i}+$ $\mathrm{n}_{2}(d)$ was introduced and related to the use of three d-orbitals by the central metal atom in bond formation. Within this bonding model, the seeming preference for unit VII in $\mathrm{Nb}_{21} \mathrm{~S}_{8}$ and unit $\mathrm{V}$ in $\mathrm{Ti}_{8} \mathrm{~S}_{3}$ can be related to the utilization of the $\mathrm{d}^{3} \mathrm{~s}^{2}$ ( $3 \mathrm{kcal} / \mathrm{mole}$ ) excited state of niobium atoms and $\mathrm{d}^{2} \mathrm{~s}^{2}$ (ground state) of titanium.

This particular approach to the bonding in the structures of the layered class suggests that the metal bonding contribution in forming Me- $\mathrm{X}$ bonds is directly related to the low lying electronic configuration of the gaseous metal atoms. In writing $\psi_{\text {bond }}$ for a particular metal in a specific phase as a combination of symmetry adapted linear combinations of atomic orbitals, the relative contribution 
of each term in the combination is related to the electronic configuration and is expressible in terms of the $n_{1}$. Within this bonding model, the $\mathrm{d}^{2} \mathrm{~s}^{2}$ ground state implies that the coefficient $(n)$ for the $\left(d^{2}\right)$, term is larger in $\psi_{\text {bond }}$ for some titanium atoms than for niobium atoms in $\mathrm{Nb}_{21} \mathrm{~S}_{8}$, etc. For niobium the $\mathrm{d}^{3} \mathrm{~s}^{2}$ exclted state $(3 \mathrm{kcal} / \mathrm{mole})$ is of low energy and the relative contribution of terms such as $\mathrm{n}_{1}\left(\mathrm{~d}^{2}\right)_{1}+\mathrm{n}_{2}(d)$ are larger than for the similar titaniumsulfide phases. In effect, similar structural units are formed from cubes and trigonal prisms for both $\mathrm{TI}_{8} \mathrm{~S}_{3}$ and $\mathrm{Nb}_{21} \mathrm{~S}_{8}$, but the structural differences. (differences in arrangement of the cubes and prisms). are related to the P.C.P. found for atoms which are the atoms forming the cubes (P.C.P. I) and prisms. It is precisely the types of P.C.P. for these atoms that correspond to the differences in units and stacking of units that can be directly related to the electronic configuration of the metal atoms involved.

This interpretation of the structural differences in $\mathrm{Nb}_{21} \mathrm{~S}_{8}$ and $\mathrm{Ti}_{8} \mathrm{~S}_{3}$ suggests an experiment that might be conducted to help support the qualitative bonding considerations presented above. If the occurrence of unft VII in $\mathrm{Nb}_{21} \mathrm{~S}_{8}$ is related to the $\mathrm{d}^{3} \mathrm{~s}^{2}$ state for niobium metal, then niobium might be expected to show a preference for occupying atom. position of both the $\mathrm{Ti}_{8} \mathrm{~S}_{3}$ and $\mathrm{Nb}_{21} \mathrm{~S}_{8}$ structure types whlch correspond to metal P.C.P. of type VII. Attempts 
could be made to prepare the two ternary phases $\mathrm{Ti}_{13} \mathrm{Nb}_{8} \mathrm{~S}_{8}$ ( $\mathrm{Nb}_{27} \mathrm{~S}_{8}$-structure type) and $\mathrm{TI}_{7} \mathrm{NbS}_{3}\left(\mathrm{Ti}_{8} \mathrm{~S}_{3}\right.$-structure type) to see if these phases are stable and if niobium does indeed occupy the predicted atom positions. 


\section{FUTURE WORK}

1. The ideas and concepts discussed here need to be tested for other structure types of the layered class. For example, comparison of the structural similarities and differences between $\mathrm{Nb}_{7} \mathrm{P}_{4}, \mathrm{Nb}_{5} \mathrm{P}_{3}$ and $\mathrm{Nb}_{8} \mathrm{P}_{5}$ would provide a better understanding of the effect of changing $\mathrm{Nb} / \mathrm{P}$ ratio on the chemical factors influencing the formation of each particular structure type. There are a number of similar comparisons which can be made for the structure types within the layered class.

2. Similar applications of the concepts discussed here should be applied to other classes of structure types. Some systems of particular interest might be the transition metal structures, the MeX structures and the $\mathrm{MeX}_{2}$ phases.

3. Of particular interest would be an investigation of possible point symmetry limitations on the bonding. interactions between two atoms. For example, the occurrence of the nonmetal trigonal prism with both parallel and perpendicular axis has been associated with certain metal P.C.P. This raises a question concerning possible symmetry correlations between the point symmetry of one atom and the polnl symmetry of 1ts neighboring atoms. 


\section{BIBLIOGRAPHY}

1. B. R. Conard, Ph.D. thesis, Iowa State University Ames, Iowa, 1969.

2. J. G. Smeggil, Ph.D. thesis, Iowa State University, Ames, Iowa, 1969.

3. M. J. Buerger, Crystal Structure Analysis, lst edition, John Wiley and Sons, Inc., New York, N.Y., 1960.

4. G. H. Stout and L. H. Jensen, X-ray Structure Determination, lst edition, The Macmillan Company, New York, N.Y., 1968.

5. M. J. Buerger, Vector Space and Its Application in Crystal-Structure Investigation, John Wiley and Sons, Inc., New York, N.Y., 1959.

6. M. M. Woolfson, An Introduction to X-ray Crystallography, Cambridge University Press, Cambridge, 1970 .

7. G. Germain and M. M. Woolfson, Acta Crystallog. B24, 91 (1968).

8. G. Germain, P. Main and M. M. Woolfson, Acta Crystallog. B26, 274 (1970).

9. G. Germain, P. Main and.M. M. Woolfson, Acta Crystallog. A27, 368 (1971).

10. J. Karle and I. L. Karie, Acta Crystallog. 2I, 849 (1966).

11. W. Biltz, P. Ehrlich and K. Meisel, Z. Anorg. Allg. Chem. 234, 97 (1937).

i2. H. F. Franzen, Ph.D. thesis, University of Kansas, 1962 .

13. G. D. Stone, M.S. thesis, University of Kansas, 1963 .

14. S. F. Bartram, Ph.D. thesis, Rutgers University, 1958 . 
15. V. N. Eremenko and V. E. Listovnichii, Khal'kogenidy (Svolstva Metody Polucheniya i Primenenie Materialov), Kiev, 1965.

16. H. Chen and H. F. Franzen, Acta Crystallog. B28, 1399 (1972).

17. International Tables for X-ray Crystallography, Vol. II, 1952).

18. D. Harker, J. Chem. Phys. 4, 38I (1936).

19. D. J. Dahm, J. E. Bensen, D. M. Nimrod, D. R. Fitzwater and R. A. Jacobson, USAEC Report IS-1701, 1967.

20. R. E. Long, Ph.D. thesis, University of California, Los Angeles, 1965.

21. E. R. Howells, D. C. Phlllips and D. Rogers, Acta Crystallog. 3, 210 (1950).

22. W. Cochran and A. S. Douglas, Proc. Roy. Soc., Ser. A A227, 486 (1957).

23. W. R. Busing, K. O. Martin and H. A. Levy, Oak Ridge National Laboratory Report ORNL-TM-305, 1962.

24. H. P. Hansen, F. Herman, J. D. Lea and S. Sk1llman, Acta Crystallog. 17, 1040 (1964).

25. H. Chen and H. F. Franzen, National Bureau of Standards Special Publication 364, Solid State Chemistry, Proceedings of the 5th Materials Research Symposium, 651 (1972).

26. $\Lambda$. Nylund, Acta Chem. Scand. 20, 2393 (1966).

27. J..P. Owens, B. R. Conard and H. F. Franzen, Acta Crystallog. 23, 77 (1967).

28: H. F. Franzen, J. Smeggil and B. R. Conard, Mat. Res. Bull. 2, 1087 (1967).

29. T. Lundström and N. Ersson, Acta Chem. Scand. 22, 1801 (1968).

30. S. Rundqvist, B. Carlsson and C. Pontchour, Acta Chem. Scand. 23, 2188 (1969). 
31. J. P. Owens and H. F. Franzen, Acta Crystallog. B30, 427 (1974).

32. H. F. Franzen, T. A. Beineke and B. R. Conard, Acta Crystallog. B24, 412 (1968).

33. H. Chen, R. T. Tuenge and H. F. Franzen, J. Inorg. Chem. 12, 552 (1973).

34. F. Grønvold, A: Kjekshus and F. Raaum, Acta Crystallog. 1.4, 930 (1961).

35. K. Selte and A. Kjekshus, Acta Chem. Scand. 17, 2560 (1963).

36. B. R. Conard, L. J. Norrby and H. F. Franzen, Acta Crystaliog. B25, 1729 (1969).

37. T. Lundström, Acta Chem. Scand. 22, 2191 (1968).

38. S. Rundqvist, Acta Chem. Scand. 20, 2427 (1966).

39. E. Hassler, Acta Chem. Scand. 25, 129 (1971).

40. S. Anugul, C. Pontchour and S. Rundqvist, Acta Chem. Scand. 27, 26 (1973).

41. T. Johnsson, Acta Chem. Scand. 26, 365 (1972).

42. O. Olofsson and E. Gangleberger, Acta Chem. Scand. 24,

43. S. Rundqvist and F. Jellinek, Acta Chem. Scand. 13, 425 (1959).

44. S. Rundqvist, Acta Chem. Scand. 14; 1961 (1960).

45. R. W. G. Wyckoff, Crystal Structures (Interscience, New York, N.Y., 1963), p. 360 .

46. B. Carlson and S. Rundqvist, Acta Chem. Scand. 25, 1742 (1971).

47. R. Berger, Acta Chem. Scand. A28, 771 (1974).

48. H. Baurecht, H. Boller and H. Nowotny, Monatsh. Chem. 101, 1696 (1970).

49. S. Rundqvist, Acta Chem. Scand. 19, 393 (1965). 
50. S. Rundqvist and A. Hede, Acta Chem. Scand. 14, 893 (1960).

51. H. F. Franzen, J. Inorg. Nucl: Chem. 28, 1575 (1966).

52. B. A. Aronsson, T. Lundström and S. Rundqvist, Borides, Silicides and Phosphides (Methuen, London, 1965).

53. L. Pauling, The Nature of the Chemlcal Bond, 3rd edition (Cornell University Press, New York, 1960).

54. J. C. Slater, J. Chem. Phys. 4I, 3199 (1964).

55. R. E. Rundle, Acta Crystallog. 1, 180 (1948).

56. N. Engel, Kem. Maanedsblad., Nos. 5, 6, 8, 9 and 10 (1949); also Powder Metallurgy Bull. I, 8 (1954).

57. L. Brewer, "Thermodynamic Stability and Bond Character in Relation to Electronic Structure and Crystal

Structure", in Electronic Structure and Alloy Chemistry of the Transition Elements, edited by P. A. Beck (Intersclence, New York, N.Y., 1963).

58. W. Hume-Rothery, Progress in Materlals Science, 13, 229 (1966).

59. S. L. Altmann, C. A. Couls on and W. Hume-Rothery, Proc. Roy. Soc., Ser. A 240, 145 (1957).

60. C. E. Moore, Atomic Energy Levels [Government Printing Office, Washington, D.C., Vol. I (1949), Vol. 2 (1952), Vol. $3(1958)$ ].

61. L. Brewer, Prediction of High Temperature Metallic Yhase Viagrams, UCRL-10701, 1963.

62. L. Brewer, Phase Stability in Metals and Alloys, edited by P. S. Rudman, J. Stringer and R. I. Jaffee (McGraw-H1ll, New York, N.Y., 1967).

63. H. F. Franzen and J. Graham, Z. Kristallogr. 123, 133 $(1966)$. 